\title{
SOME GENERALITIES ON D-MODULES IN POSITIVE CHARACTERISTIC
}

\author{
MASAharU KANEDA
}

\begin{abstract}
After the ring theoretic study of differential operators in positive characteristic by S.U. Chase and S.P. Smith, B. Haastert started investigation of $\mathcal{D}$-modules on smooth varieties in positive characteristic, and the work of R. Bøgvad followed. The purpose of this paper is to complement some basics for further study.
\end{abstract}

We fix an algebraically closed field $\mathfrak{k}$ of positive characteristic $p$. All the varieties considered in this paper will be smooth over $\mathfrak{k}$ unless otherwise specified. A celebrated theorem of A. Beilinson and J. Bernstein says that if a variety $\mathfrak{X}$ is $\mathcal{D}$-affine, then the category of $D(\mathfrak{X})$-modules is equivalent to its local version the category of $\mathcal{D}_{\mathfrak{X}}$-modules that are quasicoherent over $\mathcal{O}_{\mathfrak{x}}$. In $\S 1$ we note that the converse also holds. In $\S 2$ we will verify the base change theorem for the direct image functor of $\mathcal{D}$-modules as in characteristic 0 , that will enable us to introduce a structure of $\mathfrak{G}$-equivariant $\mathcal{D}$-module on local cohomology modules. If $\mathfrak{G}$ is an affine algebraic $\mathfrak{k}$-group acting on a variety $\mathfrak{X}$, we give in $\S 3$ an infinitesimal criterion for an $\mathcal{O}_{\mathfrak{X}}$-module to be $\mathfrak{G}$-equivariant, introduce two $\mathfrak{G}$-equivariant versions of Haastert's $\mathfrak{X}^{\infty}$ modules, and show that the equivalence in characteristic 0 of the category of Harish-Chandra (Dist $(\mathfrak{G}), \mathfrak{H})$-modules to the category of quasi- $\mathfrak{G}$-equivariant $\mathcal{D}_{\mathfrak{G} / \mathfrak{H}}$-modules carries over to positive characteristic for a closed subgroup scheme $\mathfrak{H}$ of $\mathfrak{G} . \S 4$ contains a few applications on the flag variety.

Notations. By $\mathbf{A} \mathbf{l g}_{\mathfrak{k}}$ (resp. $\mathbf{S} \mathbf{c h}_{\mathfrak{k}}$ ) we will denote the category of $\mathfrak{k}$-algebras (resp. $\mathfrak{k}$-schemes). The tensor product $\otimes$ without a subscript is always taken over $\mathfrak{k}$. If $A$ is a $\mathfrak{k}$-algebra, $A \operatorname{Mod}(\operatorname{resp} . \operatorname{Mod} A)$ will denote the category of left (resp. right) $A$-modules. If $A$ is commutative and if there is no need to distinguish left and right, the category of $A$-modules is denoted by $\mathbf{M o d}_{A}$. If there are two k-algebra homomorphisms from $A$ into $C$, one making $C$ into a left $A$-module and the other into a right $A$-module, we will call $C$ a left (resp. right) $A$-ring, and denote the category of left (resp. right) $A$-rings by $A \mathbf{R n g}$ $\left(\right.$ resp. Rng $A$ ). For a $\mathfrak{k}$-variety $\mathfrak{X}$ the category of quasicoherent $\mathcal{O}_{\mathfrak{X}}$-modules is denoted by $\mathbf{q} \mathbf{c}_{\mathfrak{X}}$, and the category of sheaves of abelian groups on $\mathfrak{X}$ by $\mathbf{A} \mathbf{b}_{\mathfrak{X}}$. If $\mathcal{A}$ is a sheaf of $\mathfrak{k}$-algebras on $\mathfrak{X}, \mathcal{A M o d}$ will denote the category of left $\mathcal{A}$-modules replacing $A$ by $\mathcal{A}$ above, and define likewise $\operatorname{Mod} \mathcal{A}$, etc. In 
case $\mathcal{A}=\mathcal{O}_{\mathfrak{X}}$, we will abbreviate $\mathcal{O}_{\mathfrak{X}}$ as $\mathfrak{X}$ and write $\mathfrak{X}$ Mod for $\mathcal{O}_{\mathfrak{X}}$ Mod, etc. The tensor product $\otimes_{\mathfrak{X}}$ will be taken over $\mathcal{O}_{\mathfrak{X}}$. The sheaf of the ring of differential operators on $\mathfrak{X}$ is denoted $\mathcal{D}_{\mathfrak{X}}$ with $D(\mathfrak{X})$ the ring of the global sections of $\mathcal{D}_{\mathfrak{X}}$, and $\mathcal{D}_{\mathfrak{X}} \mathbf{q} \mathbf{c}$ denotes the category of left $\mathcal{D}_{\mathfrak{X}}$-modules that are quasicoherent over $\mathcal{O}_{\mathfrak{X}}$. For each $r \in \mathbb{N}$ we will denote by $\mathfrak{X}^{(r)}$ the $\mathfrak{k}$-variety such that $\mathfrak{X}^{(r)}(A)=\mathfrak{X}\left(A^{(-r)}\right)$ for each $\mathfrak{k}$-algebra $A$, where $A^{(-r)}=A$ as a ring with the $\mathfrak{k}$-algebra structure given by $\xi \mapsto \xi^{p^{r}}$. Let $\mathfrak{F}_{\mathfrak{X}}^{r}: \mathfrak{X} \rightarrow \mathfrak{X}^{(r)}$ be the Frobenius morphism induced by the $\mathfrak{k}$-algebra homomorphism $A \rightarrow A^{(-r)}$ via $a \mapsto a^{p^{r}}$. If $\mathfrak{G}$ is a $\mathfrak{k}$-group, $\mathfrak{G M o d}$ will denote the category of $\mathfrak{G}$-modules. There should be no confusion with $\mathcal{O}_{\mathfrak{G}}$ Mod.

The author is grateful to B. Haastert for sending [H86], R. Bøgvad for explaining $[\mathbf{B} \varnothing]$, R. Hotta for communicating a lemma, M. Kashiwara for a suggestion, and to T. Tanisaki for consultation on the theory in characreristic 0 .

\section{$\S 1$.}

In this section $\mathfrak{X}$ will denote a smooth $\mathfrak{k}$-variety of dimension $\mathrm{N}$. We will employ the notations of [EGAIV, §16] unless otherwise specified.

1.1. The variety $\mathfrak{X}$ admits a finite affine open cover $\left(\mathfrak{X}_{i}\right)_{i}$ with each $\mathfrak{X}_{i}$ étale over the affine $\mathrm{N}$-space $\mathbb{A}_{\mathfrak{k}}^{\mathrm{N}}$. If $A$ is an étale domain over $\mathfrak{k}\left[\mathbb{A}^{\mathrm{N}}\right]$, then $A$ is flat over $\mathfrak{k}\left[\mathbb{A}^{\mathrm{N}}\right]$ and

$$
\Omega_{A / \mathfrak{k}}^{1} \simeq A \otimes \Omega_{\mathfrak{k}\left[\mathbb{A}^{N}\right] / \mathfrak{k}}^{1} \quad \text { in } A \text { Mod. }
$$

If $f: \mathfrak{k}\left[\mathbb{A}^{\mathrm{N}}\right] \rightarrow A$ is the structure homomorphism and if we write $\mathfrak{k}\left[\mathbb{A}^{\mathrm{N}}\right]=$ $\mathfrak{k}\left[t_{1}, \ldots, t_{\mathrm{N}}\right]$ with indeterminates $t_{i}, \Omega_{A / \mathfrak{k}}^{1}=\coprod_{i=1}^{\mathrm{N}} A \mathrm{~d}_{A / \mathfrak{k}} z_{i}$ with $z_{i}=f\left(t_{i}\right)$. We call $\mathbf{z}=\left(z_{1}, \ldots, z_{\mathrm{N}}\right)$ a regular system of parameters on $A$. As $A$ is smooth over $\mathfrak{k}$ [EGAIV, 16.10],

$$
\begin{aligned}
\operatorname{gr}\left(P_{A / \mathfrak{k}}\right) & \simeq S_{A}\left(\Omega_{A / \mathfrak{k}}^{1}\right) \simeq S_{A}\left(A \otimes_{\mathfrak{k}\left[\mathbb{A}^{N}\right]} \Omega_{\left.\mathfrak{k}_{\left[\mathbb{A}^{N}\right.}\right] / \mathfrak{k}}^{1}\right) \\
& \simeq A \otimes_{\mathfrak{k}\left[\mathbb{A}^{N}\right]} S_{\mathfrak{k}\left[\mathbb{A}^{N}\right] / \mathfrak{k}}\left(\Omega_{\mathfrak{k}\left[\mathbb{A}^{N}\right] / \mathfrak{k}}^{1}\right) \quad \text { as } A \text { is flat over } \mathfrak{k}\left[\mathbb{A}^{\mathrm{N}}\right] \\
& \simeq A \otimes_{\mathfrak{k}\left[\mathbb{A}^{N}\right]} \operatorname{gr}\left(P_{\mathfrak{k}\left[\mathbb{A}^{N}\right] / \mathfrak{k}}\right) .
\end{aligned}
$$

Dualizing one obtains [EGAIV, 16.11]

$$
D(A) \simeq A \otimes_{\mathfrak{k}\left[\mathbb{A}^{N}\right]} D\left(\mathbb{A}_{\mathfrak{k}}^{\mathrm{N}}\right) \quad \text { in } A \text { Mod }
$$

In particular,

$$
D(A)=\coprod_{\mathbf{n} \in \mathbb{N}^{N}} A \partial^{\mathbf{n}}=\coprod_{\mathbf{n} \in \mathbb{N}^{N}} \partial^{\mathbf{n}} A \quad \text { with } \quad \partial^{\mathbf{n}}\left(\mathbf{z}^{\mathbf{m}}\right)=\left(\begin{array}{c}
\mathbf{m} \\
\mathbf{n}
\end{array}\right) \mathbf{z}^{\mathbf{m}-\mathbf{n}} \quad \forall \mathbf{m} \in \mathbb{N}^{N},
$$


where $\mathbf{z}^{\mathbf{m}}=\prod_{i=1}^{\mathrm{N}} z_{i}^{m_{i}}$. Also [EGA0, 21.1.7]

$$
A=\coprod_{\mathbf{n} \in\left[0, p^{r}-1\right]^{\mathrm{N}}} A^{(r)} \mathbf{z}^{\mathbf{n}} \quad \forall r \in \mathbf{N},
$$

where $A^{(r)}=\left\{a^{p^{r}} \mid a \in A\right\}$. In addition to the standard filtration $\mathcal{D}$ iff $f_{\mathfrak{X}}^{n}$ there is another filtration on $\mathcal{D}_{\mathfrak{X}}$, called the $p$-filtration, defined by

$$
\mathcal{D}_{\mathfrak{X}, r}=\operatorname{Mod}_{\mathcal{O}_{\mathfrak{X}}^{(r)}}\left(\mathcal{O}_{\mathfrak{X}}, \mathcal{O}_{\mathfrak{X}}\right),
$$

where $\mathcal{O}_{\mathfrak{X}}^{(r)}$ is the sheaf of $\mathfrak{k}$-algebras such that $\mathcal{O}_{\mathfrak{X}}^{(r)}(\mathfrak{U})=\mathcal{O}_{\mathfrak{X}}(\mathfrak{U})^{(r)}$ on each open $\mathfrak{U}$ of $\mathfrak{X}[\mathbf{H 8 7}, 1.2]$. By direct computation one checks:

Lemma. If $A$ is an étale algebra over $\mathfrak{k}\left[\mathbb{A}^{1}\right]$ with a regular parameter $z$, then in $D(A)$ for any $m$ and $r \in \mathbb{N}$

$$
\left[\partial^{m}, z^{p^{r}}\right]= \begin{cases}\partial^{m-p^{r}} & \text { if } m \geq p^{r} \\ 0 & \text { otherwise }\end{cases}
$$

\subsection{One then obtains}

Proposition ([MN, 1.2.2]). For each $r \in \mathbb{N}$ the sheaf of $\mathfrak{k}$-algebras $\mathcal{D}_{\mathfrak{X}, r}$ is generated by $\mathcal{D}$ iff $f_{\mathfrak{X}}^{p^{r}-1}$ in both $\mathfrak{X R n g}$ and Rng $\mathfrak{X}$.

1.3. Let $\mathfrak{Y}$ be another smooth $\mathfrak{k}$-variety of dimension L, and let $\mathfrak{p}_{\mathfrak{X}}$ (resp. $\left.\mathfrak{p}_{\mathfrak{Y}}\right): \mathfrak{X} \times_{\mathfrak{k}} \mathfrak{Y} \rightarrow \mathfrak{X}$ (resp. $\left.\mathfrak{Y}\right)$ be the natural projection. We will suppress $\mathfrak{k}$ in $\mathfrak{X} \times_{\mathfrak{k}} \mathfrak{Y}$. If $\mathcal{M} \in \operatorname{Mod}_{\mathfrak{X}}$ and $\mathcal{N} \in \operatorname{Mod}_{\mathfrak{Y}}$, we will write $\mathcal{M} \otimes \mathcal{N}$ for $\left(\mathfrak{p}_{\mathfrak{X}}^{*} \mathcal{M}\right) \otimes_{\mathfrak{X} \times \mathfrak{Y}}\left(\mathfrak{p}_{\mathfrak{Y}}^{*} \mathcal{N}\right)$.

Proposition. There is a natural isomorphism $\mathcal{D}_{\mathfrak{X}} \otimes \mathcal{D}_{\mathfrak{Y}} \rightarrow \mathcal{D}_{\mathfrak{X} \times \mathfrak{Y}}$ under which

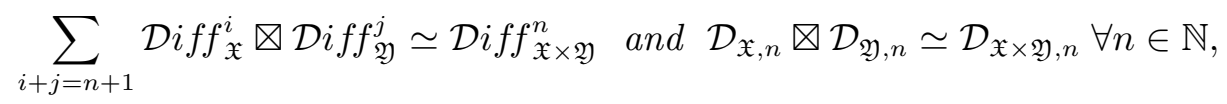

where $₫$ is taken with respect to the left $\mathcal{O}_{\mathfrak{X}}\left(\right.$ resp. $\left.\mathcal{O}_{\mathfrak{Y}}\right)$-module structure on $\mathcal{D}_{\mathfrak{X}}\left(\right.$ resp. $\left.\mathcal{D}_{\mathfrak{Y}}\right)$.

Proof. If $\mathfrak{U}$ (resp. $\mathfrak{V})$ is an affine open of $\mathfrak{X}($ resp. $\mathfrak{Y})$, then $\Gamma\left(\mathfrak{U} \times \mathfrak{V}, \mathcal{D}_{\mathfrak{X}, r} \otimes\right.$ $\left.\mathcal{D}_{\mathfrak{Y}, r}\right) \simeq D_{r}(\mathfrak{U}) \otimes D_{r}(\mathfrak{V})$ and $\Gamma\left(\mathfrak{U} \times \mathfrak{V}, \mathcal{D}_{\mathfrak{X} \times \mathfrak{Y}, r}\right) \simeq \operatorname{Mod}_{(\mathfrak{k}[\mathfrak{U}] \otimes \mathfrak{k}[\mathfrak{V}])^{(r)}}(\mathfrak{k}[\mathfrak{U}] \otimes$ $\mathfrak{k}[\mathfrak{V}], \mathfrak{k}[\mathfrak{U}] \otimes \mathfrak{k}[\mathfrak{V}])$ for each $r \in \mathbb{N}$. The natural maps $D_{r}(\mathfrak{U}) \otimes D_{r}(\mathfrak{V}) \rightarrow$ $\operatorname{Mod}_{(\mathfrak{k}[\mathfrak{U}] \otimes \mathfrak{k}[\mathfrak{V}])^{(r)}}(\mathfrak{k}[\mathfrak{U}] \otimes \mathfrak{k}[\mathfrak{V}], \mathfrak{k}[\mathfrak{U}] \otimes \mathfrak{k}[\mathfrak{V}])$ glue together to yield a morphism 
$\mathcal{D}_{\mathfrak{X}, r} \otimes \mathcal{D}_{\mathfrak{Y}, r} \rightarrow \mathcal{D}_{\mathfrak{X} \times \mathfrak{Y}, r}$. Then by taking the direct limits one obtains a morphism in $(\mathfrak{X} \times \mathfrak{Y}) \mathbf{R n g}$

$$
\mathcal{D}_{\mathfrak{X}} \otimes \mathcal{D}_{\mathfrak{Y}} \rightarrow \mathcal{D}_{\mathfrak{X} \times \mathfrak{Y}} .
$$

To see the assertions about the morphism (1), the question being local we may assume that both $\mathfrak{X}$ and $\mathfrak{Y}$ are affine and étale over $\mathbb{A}_{\mathfrak{k}}^{N}$ and $\mathbb{A}_{\mathfrak{k}}^{L}$, respectively, equipped with a regular system of parameters $\mathbf{x}$ on $A=\mathcal{O}_{\mathfrak{X}}(\mathfrak{X})$ and $\mathbf{y}$ on $C=\mathcal{O}_{\mathfrak{Y}}(\mathfrak{Y})$. The assertion about the $p$-filtrations is immediate from (1.1.4). One has (cf. [EGAIV, 16.4.23]) an isomorphism $\left(\Omega_{A / \mathfrak{k}}^{1} \otimes B\right) \oplus(A \otimes$ $\left.\Omega_{B / \mathfrak{k}}^{1}\right) \rightarrow \Omega_{A \otimes B / \mathfrak{k}}^{1}$ in $\operatorname{Mod}_{A \otimes B}$ via

$$
\left(\mathrm{d} a \otimes b, a^{\prime} \otimes \mathrm{d} b^{\prime}\right) \longmapsto(1 \otimes b) \mathrm{d}(a \otimes 1)+\left(a^{\prime} \otimes 1\right) \mathrm{d}\left(1 \otimes b^{\prime}\right) .
$$

Hence $(\mathbf{x} \otimes 1,1 \otimes \mathbf{y})=\left(x_{i} \otimes 1,1 \otimes y_{j}\right)_{i, j}$ forms a regular system of parameters on $A \otimes B$. Then for each $\mathbf{u}, \mathbf{v} \in \mathbb{N}^{N}$ and $\mathbf{p}, \mathbf{q} \in \mathbb{N}^{\mathrm{L}}$

$$
\left(\partial^{\mathbf{u}} \otimes \partial^{\mathbf{p}}\right)\left(\mathbf{x}^{\mathbf{v}} \otimes \mathbf{y}^{\mathbf{q}}\right)=\left(\begin{array}{l}
\mathbf{v} \\
\mathbf{u}
\end{array}\right)\left(\begin{array}{l}
\mathbf{q} \\
\mathbf{p}
\end{array}\right) \mathbf{x}^{\mathbf{v}-\mathbf{u}} \otimes \mathbf{y}^{\mathbf{q}-\mathbf{p}},
$$

hence $\partial^{\mathbf{u}} \otimes \partial^{\mathbf{p}} \mapsto \partial^{(\mathbf{u}, \mathbf{p})}$ under the morphism (1). Consequently one obtains the isomorphisms with respect to the standard filtrations.

1.4. By $[\mathbf{H} 87,1.3 .3,5]$

$$
\mathcal{D}_{\mathfrak{X}} \text { is not noetherian but coherent in } \mathcal{D}_{\mathfrak{X}} \mathbf{M o d} \text {, }
$$

hence a left $\mathcal{D}_{\mathfrak{X}}$-module of finite presentation type is coherent over $\mathcal{D}_{\mathfrak{X}}$. As

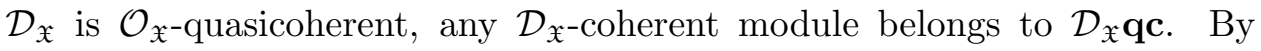
[BVI, 2.1]

$$
\mathcal{D}_{\mathfrak{X}} \mathbf{q c} \text { has enough injectives. }
$$

The following lemma was kindly communicated from Hotta R.

\section{5 .}

Lemma. An injective of $\mathcal{D}_{\mathfrak{X}} \boldsymbol{q c}$ remains injective in $\mathfrak{X M o d}$, hence flasque.

Proof. Let $\mathcal{I}$ be an injective of $\mathcal{D}_{\mathfrak{x}} \mathbf{q c}$. We first show that $\mathcal{I}$ is injective in Xqc. Given $f \in \operatorname{Mod}_{\mathfrak{X}}(\mathcal{M}, \mathcal{I})$ and a mono $j \in \operatorname{Mod}_{\mathfrak{X}}(\mathcal{M}, \mathcal{N}), \mathcal{D}_{\mathfrak{X}} \otimes_{\mathfrak{X}} j \in$ $\mathcal{D}_{\mathfrak{X}} \operatorname{Mod}\left(\mathcal{D}_{\mathfrak{X}} \otimes_{\mathfrak{X}} \mathcal{M}, \mathcal{D}_{\mathfrak{X}} \otimes_{\mathfrak{X}} \mathcal{N}\right)$ remains monic as $\mathcal{D}_{\mathfrak{X}}$ is locally free in Mod $\mathfrak{X}$. Then there is $\hat{f} \in \mathcal{D}_{\mathfrak{X}} \operatorname{Mod}\left(\mathcal{D}_{\mathfrak{X}} \otimes_{\mathfrak{X}} \mathcal{N}, \mathcal{I}\right)$ such that $\hat{f} \circ\left(\mathcal{D}_{\mathfrak{X}} \otimes_{\mathfrak{X}} j\right)=\mathcal{D}_{\mathfrak{X}} \bar{\otimes}_{\mathfrak{X}} f:$ $\delta \otimes m \mapsto \delta m$. If $\imath_{\mathcal{M}} \in \operatorname{Mod}_{\mathfrak{X}}\left(\mathcal{M}, \mathcal{D}_{\mathfrak{X}} \otimes_{\mathfrak{X}} \mathcal{M}\right)$ is the natural imbedding and likewise $\jmath_{\mathcal{N}}$, then

$$
f=\left(\mathcal{D}_{\mathfrak{X}} \bar{\otimes}_{\mathfrak{X}} f\right) \circ \imath_{\mathcal{M}}=\hat{f} \circ\left(\mathcal{D}_{\mathfrak{X}} \otimes_{\mathfrak{X}} j\right) \circ \imath_{\mathcal{M}}=\hat{f} \circ \imath_{\mathcal{N}} \circ j,
$$


hence $\mathcal{I}$ is injective in $\mathfrak{X q c}$. Next, as $\mathfrak{X}$ is noetherian, by [RD, II.7.18]

(1) there is $\mathcal{J} \in \mathfrak{X} \mathbf{q c}$, that is injective in $\mathfrak{X M o d}$,

$$
\text { and a mono } \imath \in \mathfrak{X M o d}(\mathcal{I}, \mathcal{J}) \text {. }
$$

Then $\imath$ has a left inverse $\imath^{\prime} \in \mathfrak{X M o d}(\mathcal{J}, \mathcal{I})$. Given $h \in \mathfrak{X M o d}\left(\mathcal{M}^{\prime}, \mathcal{I}\right)$ and a mono $\jmath^{\prime} \in \mathfrak{X M o d}\left(\mathcal{M}^{\prime}, \mathcal{N}^{\prime}\right)$, there is $h^{\prime} \in \mathfrak{X M o d}\left(\mathcal{N}^{\prime}, \mathcal{J}\right)$ such that $h^{\prime} \circ \jmath^{\prime}=\imath \circ h$, hence $\imath^{\prime} \circ h^{\prime} \circ \jmath^{\prime}=\imath^{\prime} \circ \imath \circ h=h$. Thus $\mathcal{I}$ remains injective in $\mathfrak{X M o d}$.

1.6. One says $\mathfrak{X}$ is $\mathcal{D}$-affine iff for each $\mathcal{M} \in \mathcal{D}_{\mathfrak{X}} \mathbf{q} \mathbf{c}$ the following two conditions hold: (i) The natural morphism $\mathcal{D}_{\mathfrak{X}} \otimes_{D(\mathfrak{X})} \mathcal{M}(\mathfrak{X}) \rightarrow \mathcal{M}$ is epic, and (ii) $\mathrm{H}^{i}(\mathfrak{X}, \mathcal{M})=0 \quad \forall i>0$. The celebrated Beilinson-Bernstein local-global principle says

(1) if $\mathfrak{X}$ is $\mathcal{D}$-affine, then $\Gamma(\mathfrak{X}, ?): \mathcal{D}_{\mathfrak{X}} \mathbf{q c} \rightarrow D(\mathfrak{X}) \mathbf{M o d}$ is an equivalence

$$
\text { of categories with quasi-inverse } \mathcal{D}_{\mathfrak{X}} \otimes_{D(\mathfrak{X})} \text { ?. }
$$

The equivalence is called the Beilinson-Bernstein correspondence. Conversely,

Proposition. If the Beilinson-Bernstein correspondence holds on $\mathfrak{X}$, then $\mathfrak{X}$ is $\mathcal{D}$-affine.

Proof. Let $\mathcal{M} \in \mathcal{D}_{\mathfrak{X}} \mathbf{q c}$. If $M=\mathcal{M}(\mathfrak{X})$ and $M \rightarrow I$ is an injective resolution in $D(\mathfrak{X})$ Mod, then $\mathcal{D}_{\mathfrak{X}} \otimes_{D(\mathfrak{X})} M \rightarrow \mathcal{D}_{\mathfrak{X}} \otimes_{D(\mathfrak{X})} I$ remains an injective resolution in $\mathcal{D}_{\mathfrak{X}} \mathbf{q} \mathbf{c}$, hence

$$
\begin{aligned}
\mathrm{H}^{i}(\mathfrak{X}, \mathcal{M}) & \simeq \mathrm{H}^{i}\left(\mathfrak{X}, \mathcal{D}_{\mathfrak{X}} \otimes_{D(\mathfrak{X})} M\right) \simeq \mathrm{H}^{i}\left(\Gamma\left(\mathfrak{X}, \mathcal{D}_{\mathfrak{X}} \otimes_{D(\mathfrak{X})} I^{\cdot}\right)\right) \quad \text { by }(1.5) \\
& \simeq \mathrm{H}^{i}\left(I^{\cdot}\right) \\
& =0 \quad \text { for } i>0 .
\end{aligned}
$$

$\S 2$.

In this section $\mathfrak{f} \in \mathbf{S c h}_{\mathfrak{k}}(\mathfrak{X}, \mathfrak{Y})$ will denote a morphism from a smooth $\mathfrak{k}$ variety $\mathfrak{X}$ of dimension $\mathrm{N}$ to another smooth $\mathfrak{k}$-variety $\mathfrak{Y}$ of dimension $\mathrm{L}$.

2.1. By a theorem of Kleiman [H, Ex. III.6.8]

(1) any coherent $\mathcal{O}_{\mathfrak{X}}$-module is the quotient of a locally free $\mathcal{O}_{\mathfrak{X}}$-module

of finite rank. 
In case $\mathfrak{X}$ is affine, by [C, Th. 3.5], [Sm, Th. 3.7]

$$
\operatorname{gldim} D(\mathfrak{X})=\operatorname{dim} \mathfrak{X} .
$$

As $\mathcal{D}_{\mathfrak{X}}$ is coherent, one obtains as in characteristic 0 (cf. [TH, Prop. I.1.4.3]):

Proposition. The category $\mathcal{D}_{\mathfrak{X}} \mathbf{q} \mathbf{c}$ has enough locally free objects. More precisely, each $\mathcal{M} \in \mathcal{D}_{\mathfrak{X}} \mathbf{q c}$ admits a resolution

$$
0 \rightarrow \mathcal{M}^{-\mathrm{N}} \rightarrow \cdots \mathcal{M}^{-1} \rightarrow \mathcal{M}^{0} \rightarrow \mathcal{M} \rightarrow 0
$$

in $\mathcal{D}_{\mathfrak{X}} \mathbf{q c}$ with $\mathcal{M}^{-i}$ locally free, $i \in[0, n-1], \mathcal{M}^{-\mathrm{N}}$ locally projective, and $\mathrm{N}=\operatorname{dim} \mathfrak{X}$. If $\mathcal{M}$ is coherent over $\mathcal{D}_{\mathfrak{X}}$, one could take all $\mathcal{M}^{i}$ coherent over $\mathcal{D}_{\mathfrak{X}}$.

2.2. The inverse image functor $\mathfrak{f}^{0}: \mathcal{D}_{\mathfrak{Y}} \mathbf{q} \mathbf{c} \rightarrow \mathcal{D}_{\mathfrak{X}} \mathbf{q c}$ is defined by $\mathfrak{f}^{0}=$ $\mathcal{D}_{\mathfrak{f} \rightarrow} \otimes_{\mathfrak{f}^{-1}} \mathcal{D}_{\mathfrak{Y}} \mathfrak{f}^{-1}$ ? with $\mathcal{D}_{\mathfrak{f} \rightarrow}=\mathfrak{f}^{*} \mathcal{D}_{\mathfrak{Y}}=\mathcal{O}_{\mathfrak{X}} \otimes_{\mathfrak{f}^{-1} \mathcal{O}_{\mathfrak{Y}}} \mathfrak{f}^{-1} \mathcal{D}_{\mathfrak{Y}} \in \mathcal{D}_{\mathfrak{X}} \operatorname{Mod}^{-1} \mathcal{D}_{\mathfrak{Y}}$. Haastert [H86, 3.6.1], however, gave another definition using the $\mathfrak{Y}^{\infty}$-module structure. If $\mathcal{M} \in \mathcal{D}_{\mathfrak{Y}} \mathbf{q} \mathbf{c}$, then $\left(\mathcal{M}^{(r)}\right)_{r \in \mathbb{N}}$ with

$$
\mathcal{M}^{(r)}=\operatorname{Mod}_{\mathcal{O}_{\mathfrak{Y}}^{(r)}}\left(\mathcal{O}_{\mathfrak{Y}}, \mathcal{O}_{\mathfrak{Y}}^{(r)}\right) \otimes_{\mathcal{D}_{\mathfrak{Y}, r}} \mathcal{M}
$$

forms a projective system of $\mathfrak{Y}^{\infty}$-module [H87, 2.2.3]. Let $\mathfrak{f}^{(r)} \in \mathbf{S c h}_{\mathfrak{k}}\left(\mathfrak{X}^{(r)}\right.$, $\left.\mathfrak{Y}^{(r)}\right)$ with $\mathfrak{f}^{(r)}(A)=\mathfrak{f}\left(A^{(-r)}\right)$ for each $A \in \mathbf{A} \lg _{\mathfrak{k}}$ so that $\mathfrak{f}^{(r)} \circ \mathfrak{F}_{\mathfrak{X}}^{r}=\mathfrak{F}_{\mathfrak{Y}}^{r} \circ \mathfrak{f}$. Then

$$
\left(\mathfrak{f}^{(r) *}\left(\mathcal{M}^{(r)}\right)\right)_{r} \text { forms an } \mathfrak{X}^{\infty} \text {-module, }
$$

hence $\varliminf_{r}\left(\mathfrak{F}_{\mathfrak{X}}^{r}\right)^{*}\left(\mathfrak{f}^{(r)}\right)^{*}\left(\mathcal{M}^{(r)}\right)$ carries a structure of $\mathcal{D}_{\mathfrak{X}} \mathbf{q} \mathbf{c}$ such that $\mathcal{D}_{\mathfrak{X}, r}$ acts on $\left(\mathfrak{F}_{\mathfrak{X}}^{r}\right)^{*}\left(\mathfrak{f}^{(r)}\right)^{*}\left(\mathcal{M}^{(r)}\right) \simeq \mathcal{O}_{\mathfrak{X}} \otimes_{\mathcal{O}_{\mathfrak{X}}^{(r)}} \mathfrak{f}^{(r) *}\left(\mathcal{M}^{(r)}\right)$ by the operation on $\mathcal{O}_{\mathfrak{X}}$.

Proposition. If $\mathcal{M} \in \mathcal{D}_{\mathfrak{Y}} \mathbf{q} \mathbf{c}$, then $\underline{\lim }\left(\mathfrak{F}_{\mathfrak{X}}^{r}\right)^{*}\left(\mathfrak{f}^{(r)}\right)^{*}\left(\mathcal{M}^{(r)}\right) \simeq \mathcal{D}_{\mathfrak{f} \rightarrow} \otimes_{\mathfrak{f}-1} \mathcal{D}_{\mathfrak{Y}}$ $\mathfrak{f}^{-1} \mathcal{M}$ in $\mathcal{D}_{\mathfrak{x}} \mathbf{q} \mathbf{c}$, hence the two definitions agree.

Proof. It is enough to show

$$
\left(\mathfrak{F}_{\mathfrak{X}}^{r}\right)^{*}\left(\mathfrak{f}^{(r)}\right)^{*}\left(\mathcal{M}^{(r)}\right) \simeq \mathcal{D}_{\mathfrak{f} \rightarrow, r} \otimes_{\mathfrak{f}^{-1}} \mathcal{D}_{\mathfrak{Y}, r} \mathfrak{f}^{-1} \mathcal{M} \quad \text { in } \mathcal{D}_{\mathfrak{X}, r} \text { Mod }
$$

where $\mathcal{D}_{\mathfrak{f} \rightarrow, r}=\mathfrak{f}^{*}\left(\mathcal{D}_{\mathfrak{Y}, r}\right)$. One has in $\mathfrak{X M o d}$

(3) $\left(\mathfrak{F}_{\mathfrak{X}}^{r}\right)^{*}\left(\mathfrak{f}^{(r)}\right)^{*}\left(\mathcal{M}^{(r)}\right) \simeq \mathfrak{f}^{*}\left(\mathfrak{F}_{\mathfrak{Y}}^{r}\right)^{*}\left(\mathcal{M}^{(r)}\right) \simeq \mathfrak{f}^{*} \mathcal{M} \simeq \mathcal{D}_{f \rightarrow, r} \otimes_{\mathfrak{f}^{-1}} \mathcal{D}_{\mathfrak{Y}, r} \mathfrak{f}^{-1} \mathcal{M}$. 
To check that the composite isomorphism is $\mathcal{D}_{\mathfrak{Y}, r}$-equivariant, we may assume $\mathfrak{X}$ and $\mathfrak{Y}$ are both affine. If $A=\mathcal{O}_{\mathfrak{X}}(\mathfrak{X}), C=\mathcal{O}_{\mathfrak{Y}}(\mathfrak{Y})$, and if $M=$ $\mathcal{M}(\mathfrak{X})$, then the isomorphisms (3) read

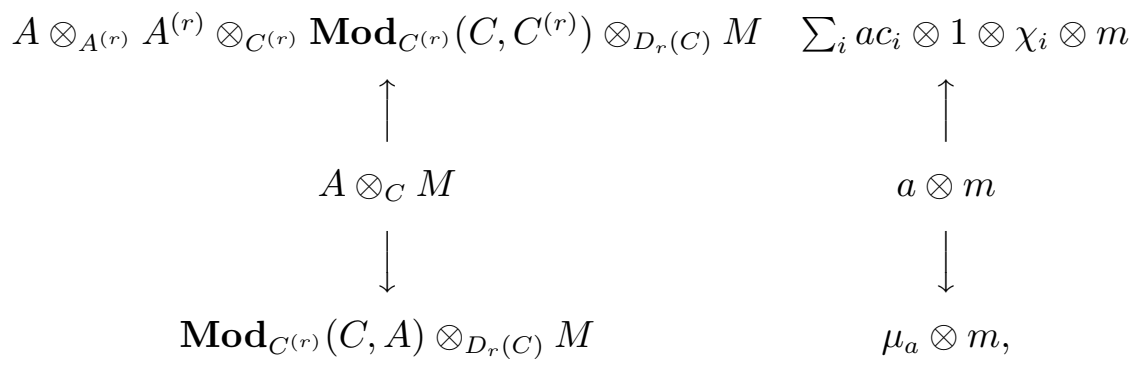

where $\sum_{i} c_{i} \otimes \chi_{i} \mapsto \mathrm{id}_{C}$ under the bijection $C \otimes_{C^{(r)}} \operatorname{Mod}_{C^{(r)}}\left(C, C^{(r)}\right) \simeq$ $D_{r}(C)$, and $\mu_{a}$ is the multplication by $a$.

If $\delta \in D_{r}(A)$, then $\delta \cdot \sum_{i} a c_{i} \otimes 1 \otimes \chi_{i} \otimes m=\sum_{i} \delta\left(a c_{i}\right) \otimes 1 \otimes \chi_{i} \otimes m$ while $\delta \cdot\left(\mu_{a} \otimes m\right)=\left(\delta \circ \mu_{a}\right) \otimes m$, hence we must check $\sum_{i} \mu_{\delta\left(a c_{i}\right)} \circ \chi_{i}=\delta \circ \mu_{a}$ in $\operatorname{Mod}_{C^{(r)}}(C, A)$. If $c \in C$, then

$$
\begin{aligned}
\sum_{i}\left(\mu_{\delta\left(a c_{i}\right)} \circ \chi_{i}\right)(c) & =\sum_{i} \delta\left(a c_{i}\right) \chi_{i}(c)=\sum_{i} \delta\left(a c_{i} \chi_{i}(c)\right)=\delta\left(a \sum_{i} c_{i} \chi_{i}(c)\right) \\
& =\delta(a c)=\left(\delta \circ \mu_{a}\right)(c)
\end{aligned}
$$

as desired.

2.3. Let $\omega_{\mathfrak{X}}=\wedge^{\mathrm{N}} \Omega_{\mathfrak{X} / \mathfrak{k}}^{1}, \omega_{\mathfrak{Y}}=\wedge^{\mathrm{L}} \Omega_{\mathfrak{Y} / \mathfrak{k}}^{1}$, and set

$$
\mathcal{D}_{\mathfrak{f} \leftarrow}=\mathfrak{f}^{*}\left(\mathcal{D}_{\mathfrak{Y}} \otimes_{\mathfrak{Y}} \omega_{\mathfrak{Y}}^{-1}\right) \otimes_{\mathfrak{X}} \omega_{\mathfrak{X}} \simeq\left(\mathcal{D}_{\mathfrak{f} \rightarrow} \otimes_{\mathfrak{f}^{-1}} \mathcal{D}_{\mathfrak{Y}} \mathfrak{f}^{-1}\left(\mathcal{D}_{\mathfrak{Y}} \otimes_{\mathfrak{Y}} \omega_{\mathfrak{Y}}^{-1}\right)\right) \otimes_{\mathfrak{X}} \omega_{\mathfrak{X}} .
$$

The module $\mathcal{D}_{\mathfrak{f} \leftarrow}$ carries a structure of $\mathfrak{f}^{-1} \mathcal{D}_{\mathfrak{Y}} \mathbf{M o d} \mathcal{D}_{\mathfrak{X}}[\mathbf{H} 88,7.1]$. To describe the structure locally, let $\mathfrak{V}$ (resp. $\mathfrak{U} \subseteq \mathfrak{f}^{-1} \mathfrak{V}$ ) be an affine open of $\mathfrak{Y}$ (resp. $\mathfrak{X}$ )

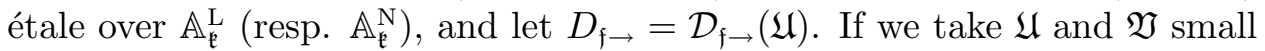
enough that $\mathcal{D}_{\mathfrak{f} \leftarrow}(\mathfrak{U})$ may be identified with $D_{\mathfrak{f} \rightarrow}$ as $\mathfrak{k}$-linear spaces, then

$$
\delta_{1} \cdot \theta \cdot \delta_{2}=\delta_{2}^{*} \theta \delta_{1}^{*}, \quad \delta_{1} \in D(\mathfrak{V}), \delta_{2} \in D(\mathfrak{U}), \theta \in D_{\mathfrak{f} \rightarrow,}
$$

using the structure of $\mathcal{D}_{\mathfrak{X}} \mathbf{M o d}^{-1} \mathcal{D}_{\mathfrak{Y}}$ on $\mathcal{D}_{\mathfrak{f} \rightarrow \text {, where }}{ }^{*}: D(\mathfrak{U}) \rightarrow D(\mathfrak{U})$ is an involutive antiautomorphism of $\mathfrak{k}$-algebras such that $\sum a_{\mathbf{n}} \partial^{\mathbf{n}} \mapsto$ $\sum(-1)^{|\mathbf{n}|} \partial^{\mathbf{n}} a_{\mathbf{n}}, a_{\mathbf{n}} \in A=\mathcal{O}_{\mathfrak{X}}(\mathfrak{U}),|\mathbf{n}|=\sum_{i=1}^{\mathrm{N}} n_{i}[\mathbf{H} 88,5.5]$. More explicitly, if $D_{\mathfrak{f} \rightarrow, r}=\mathcal{D}_{\mathfrak{f} \rightarrow, r}(\mathfrak{U})$ and $D_{\mathfrak{f} \leftarrow, r}=\mathcal{D}_{\mathfrak{f} \leftarrow, r}(\mathfrak{U})$ with $\mathcal{D}_{\mathfrak{f} \leftarrow, r}=\mathfrak{f}^{*}\left(\mathcal{D}_{\mathfrak{Y}, r} \otimes_{\mathfrak{Y}} \omega_{\mathfrak{Y}}^{-1}\right) \otimes_{\mathfrak{X}} \omega_{\mathfrak{X}}$ and if $C=\mathcal{O}_{\mathfrak{Y}}(\mathfrak{V})$, then $D_{\mathfrak{f} \leftarrow, r}$ can be identified with $D_{\mathfrak{f} \rightarrow, r} \simeq \operatorname{Mod}_{C^{(r)}}(C, A)$ as $\mathfrak{k}$-linear spaces with the $D_{r}(C) \operatorname{Mod} D_{r}(A)$-structure given by

$$
\delta_{1} \cdot \theta \cdot \delta_{2}=\delta_{2}^{*} \circ \theta \circ \delta_{1}^{*} \forall \delta_{1} \in D_{r}(C), \delta_{2} \in D_{r}(A), \theta \in \operatorname{Mod}_{C^{(r)}}(C, A) .
$$


Both $D_{r}(C)$ and $D_{r}(A)$ are invariant under ${ }^{*}$ by (1.2). Hence if $\mathcal{M} \in \mathcal{D}_{\mathfrak{x}} \mathbf{q c}$, $\mathcal{D}_{\mathfrak{f} \leftarrow} \otimes_{\mathcal{D}_{\mathfrak{X}}} \mathcal{M}$ carries a structure of left $\mathfrak{f}^{-1} \mathcal{D}_{\mathfrak{Y}}$-module. Define a morphism of ringed spaces $\mathfrak{f}_{0}:\left(\mathfrak{X}, \mathfrak{f}^{-1} \mathcal{D}_{\mathfrak{Y}}\right) \rightarrow\left(\mathfrak{Y}, \mathcal{D}_{\mathfrak{Y}}\right)$ via $\mathcal{N} \mapsto \mathfrak{f}_{*} \mathcal{N}$. Then the direct image of $\mathcal{M}$ under $\mathfrak{f}$ is defined by $\mathfrak{f}_{0}\left(\mathcal{D}_{\mathfrak{f} \leftarrow} \otimes_{\mathcal{D}_{\mathfrak{X}}} \mathcal{M}\right)$. If one defines $\mathfrak{f}_{0, r}$ : $\left(\mathfrak{X}, \mathfrak{f}^{-1} \mathcal{D}_{\mathfrak{Y}, r}\right) \rightarrow\left(\mathfrak{Y}, \mathcal{D}_{\mathfrak{Y}, r}\right)$ likewise, then $\mathfrak{f}_{0}\left(\mathcal{D}_{\mathfrak{f} \leftarrow} \otimes_{\mathcal{D}_{\mathfrak{X}}} \mathcal{M}\right) \simeq \underline{\lim }_{0, r}\left(\mathcal{D}_{\mathfrak{f} \leftarrow, r} \otimes_{\mathcal{D}_{\mathfrak{X}, r}}\right.$

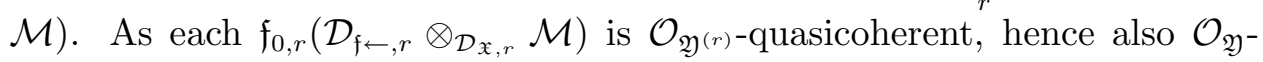
quasicoherent $[\mathbf{H} 88,3.1]$,

$$
\mathfrak{f}_{0}\left(\mathcal{D}_{\mathfrak{f} \leftarrow} \otimes_{\mathcal{D}_{\mathfrak{X}}} \mathcal{M}\right) \in \mathcal{D}_{\mathfrak{Y}} \mathbf{q} \mathbf{c} .
$$

One then defines the derived direct image functor by

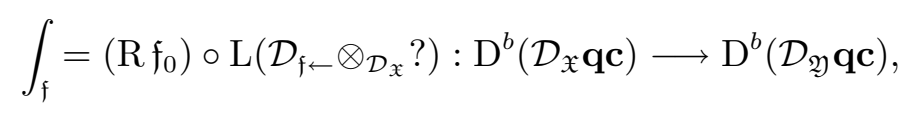

where $\mathrm{D}^{b}$ denotes the bounded derived category. There is a simplification due to $[\mathbf{H 8 8}, 1.2]$ that

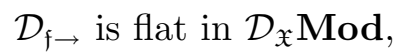

hence also $[\mathbf{H 8 8}, 7.2]$

$$
\mathcal{D}_{\mathfrak{f} \leftarrow} \text { is flat in } \operatorname{Mod} \mathcal{D}_{\mathfrak{X}} \text {. }
$$

Consequently,

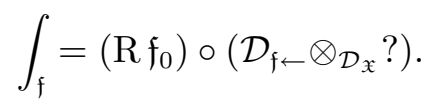

To see that $\int_{\mathfrak{f}}$ actually lands in $\mathrm{D}^{b}\left(\mathcal{D}_{\mathfrak{Y}} \mathbf{q} \mathbf{c}\right)$, however, seems to the present author to require as in [BVI, 5.1] a spectral sequence argument and J. Bernstein's theorem [BVI, 2.10] that if $\mathrm{D}_{q c}^{b}\left(\mathcal{D}_{\mathfrak{X}} \mathbf{M o d}\right)$ is the derived category of bounded complexes of left $\mathcal{D}_{\mathfrak{X}}$-modules with $\mathcal{O}_{\mathfrak{X}}$-quasicoherent cohomlogies, then

(7) the imbedding of $\mathcal{D}_{\mathfrak{X}} \mathbf{q} \mathbf{c}$ into $\mathcal{D}_{\mathfrak{X}} \mathbf{M o d}$ induces an equivalence

$$
\mathrm{D}^{b}\left(\mathcal{D}_{\mathfrak{X}} \mathbf{q} \mathbf{c}\right) \rightarrow \mathrm{D}_{q c}^{b}\left(\mathcal{D}_{\mathfrak{X}} \mathbf{M o d}\right) .
$$

As we will need the argument in the proof of the base change theorem, let us recall the spectral sequence from [BVI, 5.1].

2.4. Let $\mathcal{U}=\left\{\mathfrak{X}_{i} \mid i \in I\right\}$ be a finite affine open covering of $\mathfrak{X}$. We number $I$, and for each $J=\left(i_{0}, \ldots, i_{r}\right) \in I^{r+1}$ with $i_{0}<\cdots<i_{r}$ let $\mathfrak{X}_{J}=\cap_{j=0}^{r} \mathfrak{X}_{i_{j}}, \mathfrak{i}_{J}: \mathfrak{X}_{J} \hookrightarrow \mathfrak{X}$, and $\mathfrak{f}_{J}=\mathfrak{f} \circ \mathfrak{i}_{J}$. If $\mathcal{A}$ is a sheaf of abelian 
groups on $\mathfrak{X}$, let $\mathcal{A} \rightarrow \mathcal{F}$ be a flasque resolution, and for each $t \in \mathbb{N}$ let $\mathcal{F}^{t} \rightarrow \mathcal{C} \cdot\left(\mathcal{U}, \mathcal{F}^{t}\right)$ be the Cech resolution of $\mathcal{F}^{t}[\mathbf{H}$, II.4]. Then from the double complex $\mathfrak{f}_{*} \mathcal{C} \cdot(\mathcal{U}, \mathcal{F} \cdot)=\bigsqcup_{s, t} \mathfrak{f}_{*} \mathcal{C}^{s}\left(\mathcal{U}, \mathcal{F}^{t}\right)$ one obtains:

Lemma. If $\mathcal{A}$ is a sheaf of abelian groups on $\mathfrak{X}$, there is a spectral sequence

$$
\mathrm{E}_{1}^{s, t}=\prod_{|J|=s+1}\left(\mathrm{R}^{t}\left(\mathfrak{f}_{J}\right)_{*}\right)\left(\left.\mathcal{A}\right|_{\mathfrak{X}_{J}}\right) \Rightarrow\left(\mathrm{R}_{\mathfrak{f}_{*}}\right)(\mathcal{A}) .
$$

2.5. Just like on $\mathbf{q c}_{\mathfrak{X}}$ one has (cf. [TH, Th. I.1.8.2]):

Proposition (Base change). Given a cartesian square

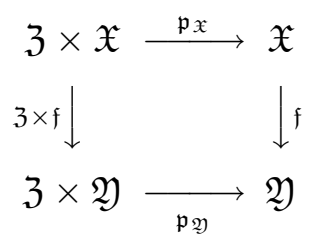

one has on $\mathrm{D}^{b}\left(\mathcal{D}_{\mathfrak{X}} \mathbf{q} \mathbf{c}\right)$

$$
\mathfrak{p}_{\mathfrak{Y}}^{0} \circ \int_{\mathfrak{f}} \simeq\left(\mathrm{L} \mathfrak{p}_{\mathfrak{Y}}^{0}\right) \circ \int_{\mathfrak{f}} \simeq \int_{\mathfrak{Z} \times \mathfrak{f}} \circ\left(\mathrm{L} \mathfrak{p}_{\mathfrak{X}}^{0}\right) \simeq \int_{\mathfrak{Z} \times \mathfrak{f}} \circ\left(\mathfrak{p}_{\mathfrak{X}}^{0}\right)
$$

Proof. As $\mathfrak{p}_{\mathfrak{X}}$ is flat, $\mathrm{L} \mathfrak{p}_{\mathfrak{X}}^{0} \simeq \mathfrak{p}_{\mathfrak{X}}^{0}$, and likewise for $\mathfrak{p}_{\mathfrak{Y}}$. Let $\mathcal{M} \in \mathcal{D}_{\mathfrak{X}} \mathbf{q c}$. Take a locally free resolution $\mathcal{L} \rightarrow \mathcal{M}$ in $\mathcal{D}_{\mathfrak{X}} \mathbf{q c}$ and a finite affine open cover $\left(\mathfrak{X}_{i}\right)_{i}$ of $\mathfrak{X}$ such that $\left.\mathcal{L}^{t}\right|_{\mathfrak{x}_{i}}$ is free for all $t \in\{0,-1\}$ and $i \in I$. As $\mathcal{D}_{\mathfrak{f} \leftarrow}$ is flat in $\operatorname{Mod} \mathcal{D}_{\mathfrak{X}}$, the sequence $\mathcal{D}_{\mathfrak{f} \leftarrow} \otimes_{\mathcal{D}_{\mathfrak{X}}} \mathcal{L} \rightarrow \mathcal{D}_{\mathfrak{f} \leftarrow} \otimes_{\mathcal{D}_{\mathfrak{X}}} \mathcal{M}$ remains exact. By the

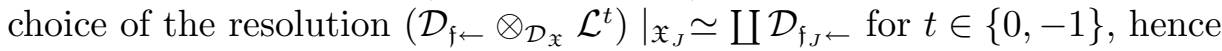

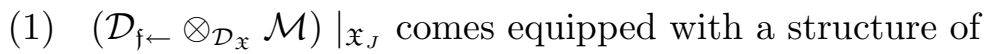

$$
\text { right } \mathcal{O}_{\mathfrak{X}} \text {-quasicoherent module. }
$$

As $\mathfrak{f}^{-1} \mathcal{D}_{\mathfrak{Y}}$ Mod has enough injectives, that are flasque (cf. [G, II.7.1]), one can compute $\mathrm{R} \mathfrak{f}_{0}$ with flasques, hence by (1) and Serre's theorem one obtains from (2.4) a spectral sequence

$$
\begin{aligned}
\mathrm{E}_{1}^{s, t} & = \begin{cases}\prod_{|J|=s+1}\left(\mathfrak{f}_{J}\right)_{0}\left(\left.\left(\mathcal{D}_{\mathfrak{f} \leftarrow} \otimes_{\mathcal{D}_{\mathfrak{X}}} \mathcal{M}\right)\right|_{\mathfrak{X}_{J}}\right)=\prod_{J} \int_{\mathfrak{f}_{J}}^{0} \mathcal{M}_{J} & \text { if } t=0 \\
0 & \text { otherwise }\end{cases} \\
& \Longrightarrow\left(\mathrm{R}_{\mathfrak{f}_{0}}\right)\left(\mathcal{D}_{\mathfrak{f} \leftarrow} \otimes_{\mathcal{D}_{\mathfrak{X}}} \mathcal{M}\right),
\end{aligned}
$$


where $\mathcal{M}_{J}=\left.\mathcal{M}\right|_{\mathfrak{X}_{J}}$. One then obtains

$$
\int_{\mathfrak{f}} \mathcal{M} \simeq \mathrm{H}^{\cdot}\left(\prod_{|J|=s+1} \int_{\mathfrak{f}_{J}}^{0} \mathcal{M}_{J}\right),
$$

and likewise

$$
\int_{\mathfrak{Z} \times \mathfrak{f}} \mathfrak{p}_{\mathfrak{X}}^{0}(\mathcal{M}) \simeq \mathrm{H}^{\cdot}\left(\prod_{|J|=s+1} \int_{\mathfrak{Z} \times \mathfrak{f}_{J}}^{0} \mathfrak{p}_{\mathfrak{X}_{J}}^{0}\left(\mathcal{M}_{J}\right)\right)
$$

As $\mathfrak{p}_{\mathfrak{Y}}^{0}$ is exact, the isomorphism (3) induces

$$
\mathfrak{p}_{\mathfrak{Y}}^{0}\left(\int_{\mathfrak{f}} \mathcal{M}\right) \simeq \mathrm{H}^{\cdot}\left(\prod_{|J|=s+1} \mathfrak{p}_{\mathfrak{Y}}^{0}\left(\int_{\mathfrak{f}_{J}}^{0} \mathcal{M}_{J}\right)\right)
$$

Moreover, for $t \in\{0,-1\}$ one has in $\operatorname{Mod}_{\mathfrak{Z} \times \mathfrak{X}_{J}}$

$$
\begin{aligned}
\mathcal{D}_{\mathfrak{Z} \times \mathfrak{f}_{J} \leftarrow} \otimes_{\mathcal{D}_{\mathfrak{Z} \times \mathfrak{X}_{J}}} \mathfrak{p}_{\mathfrak{X}_{J}}^{0}\left(\left.\mathcal{L}^{t}\right|_{\mathfrak{X}_{J}}\right) \simeq \coprod \mathcal{D}_{\mathfrak{Z} \times \mathfrak{f}_{J} \leftarrow} \otimes_{\mathcal{D}_{\mathfrak{3}} \otimes \mathcal{D}_{\mathfrak{X}_{J}}}\left(\mathcal{O}_{\mathfrak{Z}} \otimes \mathcal{D}_{\mathfrak{X}_{J}}\right) \\
\simeq \coprod\left(\mathcal{D}_{\mathfrak{Z}} \otimes \mathcal{D}_{\mathfrak{f}_{J} \leftarrow}\right) \otimes_{\mathfrak{p}_{\mathfrak{J}}^{*} \mathcal{D}_{\mathfrak{Z}}} \mathcal{O}_{\mathfrak{Z} \times \mathfrak{X}_{J}} \simeq \coprod\left(\mathcal{O}_{\mathfrak{Z}} \otimes \mathcal{D}_{\mathfrak{f}_{J} \leftarrow}\right),
\end{aligned}
$$

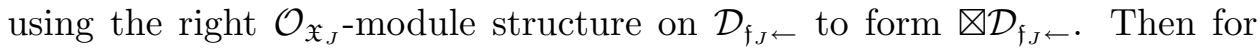
each $r \in \mathbb{N}$ one obtains in $\mathcal{D}_{\mathfrak{Z} \times \mathfrak{Y}, r} \mathbf{M o d}$

$$
\left(\mathfrak{Z} \times \mathfrak{f}_{J}\right)_{0} \mathfrak{p}_{\mathfrak{X}_{J}}^{0}\left(\mathcal{D}_{\mathfrak{f}_{J} \leftarrow, r}\right) \simeq \mathfrak{p}_{\mathfrak{Y}}^{0}\left(\mathfrak{f}_{J}\right)_{0}\left(\mathcal{D}_{\mathfrak{f}_{J} \leftarrow, r}\right),
$$

hence in $\mathcal{D}_{\mathfrak{Z} \times \mathfrak{Y}}$ Mod

$$
\begin{aligned}
& \int_{\mathfrak{Z} \times \mathfrak{f}_{J}}^{0} \mathfrak{p}_{\mathfrak{X}_{J}}^{0}\left(\left.\mathcal{L}^{t}\right|_{\mathfrak{X}_{J}}\right) \simeq \coprod \underset{r}{\lim }\left(\mathfrak{Z} \times \mathfrak{f}_{J}\right)_{0} \mathfrak{p}_{\mathfrak{X}_{J}}^{0}\left(\mathcal{D}_{\mathfrak{f}_{J} \leftarrow, r}\right) \\
& \simeq \coprod \underset{r}{\lim _{\mathfrak{Y}}} \mathfrak{p}_{\mathfrak{Y}}^{0}\left(\mathfrak{f}_{J}\right)_{0}\left(\mathcal{D}_{\mathfrak{f}_{J} \leftarrow, r}\right) \simeq \mathfrak{p}_{\mathfrak{Y}}^{0} \int_{\mathfrak{f}_{J}}^{0}\left(\left.\mathcal{L}^{t}\right|_{\mathfrak{X}_{J}}\right) .
\end{aligned}
$$

As $\mathcal{D}_{\mathfrak{Z} \times \mathfrak{f}_{J} \leftarrow}$ is flat in $\operatorname{Mod} \mathcal{D}_{\mathfrak{Z} \times \mathfrak{X}_{J}}, \quad \mathcal{D}_{\mathfrak{Z} \times \mathfrak{f}_{J} \leftarrow} \otimes_{\mathcal{D}_{\mathfrak{Z} \times \mathfrak{X}_{J}}} \mathfrak{p}_{\mathfrak{X}_{J}}^{0}\left(\left.\mathcal{L}\right|_{\mathfrak{X}_{J}}\right) \rightarrow$ $\mathcal{D}_{\mathfrak{Z} \times \mathfrak{f}_{J} \leftarrow} \otimes_{\mathcal{D}_{\mathfrak{Z} \times \mathfrak{X}_{J}}} \mathfrak{p}_{\mathfrak{X}_{J}}^{0}\left(\mathcal{M}_{J}\right)$ remains exact, hence $\mathcal{D}_{\mathfrak{Z} \times \mathfrak{f}_{J} \leftarrow} \otimes_{\mathcal{D}_{\mathfrak{Z} \times \mathfrak{X}_{J}}} \mathfrak{p}_{\mathfrak{X}_{J}}^{0}\left(\mathcal{M}_{J}\right)$ comes equipped with a structure of right $\mathcal{O}_{\mathfrak{Z} \times \mathfrak{X}_{J}}$-quasicoherent module. Then $\int_{\mathfrak{Z} \times \mathfrak{f}_{J}}^{0} \mathfrak{p}_{\mathfrak{X}_{J}}^{0}\left(\left.\mathcal{L} \cdot\right|_{\mathfrak{X}_{J}}\right) \rightarrow \int_{\mathfrak{Z} \times \mathfrak{f}_{J}}^{0} \mathfrak{p}_{\mathfrak{X}_{J}}^{0}\left(\mathcal{M}_{J}\right)$ is still exact by Serre's theorem, and likewise $\mathfrak{p}_{\mathfrak{Y}}^{0} \int_{\mathfrak{f}_{J}}^{0}\left(\left.\mathcal{L} \cdot\right|_{\mathfrak{X}_{J}}\right) \rightarrow \mathfrak{p}_{\mathfrak{Y}}^{0} \int_{\mathfrak{f}_{J}}^{0} \mathcal{M}_{J}$. Hence

$$
\int_{\mathfrak{Z} \times \mathfrak{f}_{J}}^{0} \mathfrak{p}_{\mathfrak{X}_{J}}^{0}\left(\mathcal{M}_{J}\right) \simeq \mathfrak{p}_{\mathfrak{Y}}^{0} \int_{\mathfrak{f}_{J}}^{0} \mathcal{M}_{J} \quad \text { in } \mathcal{D}_{\mathfrak{Z} \times \mathfrak{Y}} \text { Mod }
$$


The assertion now follows from (4) and (5).

2.6. If $\mathfrak{f}: \mathfrak{X} \rightarrow \mathfrak{Y}$ is an open immersion,

$$
\mathcal{D}_{\mathfrak{f} \rightarrow} \simeq \mathcal{D}_{\mathfrak{x}} \quad \text { and } \quad \mathcal{D}_{\mathfrak{f} \leftarrow} \simeq \mathcal{D}_{\mathfrak{x}}^{\prime} \quad \text { both in } \mathcal{D}_{\mathfrak{X}} \operatorname{Mod} \mathcal{D}_{\mathfrak{X}}
$$

where $\mathcal{D}_{\mathfrak{X}}^{\prime}$ is $\mathcal{D}_{\mathfrak{X}}$ in $\mathbf{A} \mathbf{b}_{\mathfrak{X}}$ but the $\mathcal{D}_{\mathfrak{X}} \mathbf{M o d} \mathcal{D}_{\mathfrak{X}}$-structure given by the formula (2.3.1). Hence:

Proposition. If $\mathfrak{f}$ is an open immersion, then $\mathrm{L} \mathfrak{f}^{0} \simeq \mathfrak{f}^{-1}$ and $\int_{\mathfrak{f}} \simeq \mathrm{R} \mathfrak{f}_{0}$.

2.7. Assume $\mathfrak{f}$ is étale. As an open immersion is étale, for the local study of $\mathfrak{f}$ we may assume both $\mathfrak{X}$ and $\mathfrak{Y}$ are affine. Thus let $A=\mathcal{O}_{\mathfrak{X}}(\mathfrak{X})$ and $C=$ $\mathcal{O}_{\mathfrak{Y}}(\mathfrak{Y})$. If $\left(z_{i}\right)_{1 \leq i \leq N}$ is a regular system of parameters on $C$, then $\left(\mathfrak{f}^{\circ}\left(z_{i}\right)\right)_{i}$ forms one on $A$ and the isomorphism (1.1.2) generalizes to an isomorphism in $A$ Mod

$$
A \otimes_{C} D(C) \rightarrow D(A) \quad \text { via } \quad a \otimes \partial_{C}^{\mathbf{n}} \longmapsto a \partial_{A}^{\mathbf{n}},
$$

where $\partial_{A}^{\mathbf{n}} \in D(A)$ (resp. $\left.\partial_{C}^{\mathbf{n}} \in D(C)\right)$ are as defined in (1.1.3).

Proposition. Assume $\mathfrak{f}: \mathfrak{X} \rightarrow \mathfrak{Y}$ is an étale morphism of affine varieties with $A=\mathcal{O}_{\mathfrak{X}}(\mathfrak{X})$ and $C=\mathcal{O}_{\mathfrak{Y}}(\mathfrak{Y})$.

(i) For each $r \in \mathbb{N}$ the bijection (1) induces an A-module isomorphism

$$
A \otimes_{C} D_{r}(C) \longrightarrow D_{r}(A) \quad \text { via } \quad a \otimes\left[\left(c_{\mathbf{n m}}\right)\right] \longmapsto a\left[\left(\mathfrak{f}^{\circ}\left(c_{\mathbf{n m}}\right)\right)\right],
$$

where $D_{r}(A)$ (resp. $\left.D_{r}(C)\right)$ is identified with the $p^{r \mathrm{~N}} \times p^{r \mathrm{~N}}$ matrix algebra over $A^{(r)}$ (resp. $\left.C^{(r)}\right)$. Hence another induced map $D\left(\mathfrak{f}^{\circ}\right)$ : $D(C) \rightarrow D(A)$ such that $c \partial_{C}^{\mathrm{n}} \mapsto \mathfrak{f}^{\mathrm{o}}(c) \partial_{A}^{\mathrm{n}}$ is a $\mathfrak{k}$-algebra homomorphism.

(ii) If $\mathcal{M} \in \mathcal{D}_{\mathfrak{X}} \mathbf{q} \mathbf{c}$, then $\left(\int_{\mathfrak{f}}^{0} \mathcal{M}\right)(\mathfrak{Y}) \simeq \mathcal{M}(\mathfrak{X})$ in $D(C)$ Mod with $D(C)$ acting on $\mathcal{M}(\mathfrak{X})$ via $D\left(\mathfrak{f}^{\circ}\right)$.

Proof. Define $A \otimes_{C} D_{r}\left(\mathfrak{f}^{\circ}\right) \in A \operatorname{Mod}\left(A \otimes_{C} D_{r}(C), D_{r}(A)\right)^{\times}$via $a \otimes\left[\left(c_{\mathbf{n m}}\right)\right] \mapsto$ $a\left[\left(\mathfrak{f}^{\mathrm{o}}\left(c_{\mathbf{n m}}\right)\right)\right]$. If $\mathbf{t} \in \mathbb{N}^{\mathrm{N}}$, take $r \gg 0$ that $\partial_{C}^{\mathbf{t}} \in D_{r}(C)$. As $\partial_{C}^{\mathbf{t}}$ is determined by the evaluations at $\mathbf{z}^{n}, \mathbf{n} \leq \mathbf{t}$, if $e_{\mathbf{n m}}^{C} \in D_{r}(C)$ with $e_{\mathbf{n m}}^{C}\left(\mathbf{z}^{\mathbf{l}}\right)=\delta_{\mathbf{m l}} \mathbf{z}^{\mathbf{n}}$, then

$$
\partial_{C}^{\mathbf{t}} \in \sum_{\mathbf{n}, \mathbf{m} \in\left[0, p^{r}-1\right]^{\mathrm{N}}} \mathfrak{k}[\mathbf{z}] e_{\mathbf{n m}}^{C} .
$$

Then $\left(A \otimes_{C} D_{r}\left(\mathfrak{f}^{\circ}\right)\right)\left(\partial_{C}^{\mathbf{t}}\right)=\partial_{A}^{\mathbf{t}}$, hence $\underline{\lim }\left(A \otimes_{C} D_{r}\left(\mathfrak{f}^{\circ}\right)\right)$ coincides with the map (1), and (i) follows. Also we obtain a bijection $D_{\mathfrak{f} \leftarrow, r} \simeq A \otimes_{C} D_{r}(C) \simeq$ 
$D_{r}(A)$, under which the $D_{r}(C) \operatorname{Mod} D_{r}(A)$-structure on $D_{\mathfrak{f} \leftarrow, r}$ is transferred to $D_{r}(A)$ such that

$$
\left(c \partial_{C}^{\mathbf{n}}\right) \cdot \delta \cdot \delta_{A}=\delta_{A}^{*} \circ \delta \circ(-1)^{|\mathbf{n}|} \partial_{A}^{\mathbf{n}} \mathfrak{f}^{\circ}(c)=\delta_{A}^{*} \circ \delta \circ\left(\mathfrak{f}^{\circ}(c) \partial_{A}^{\mathbf{n}}\right)^{*} .
$$

Then in $D_{r}(C)$ Mod

$$
\left(\int_{\mathfrak{f}, r}^{0} \mathcal{M}\right)(\mathfrak{Y}) \simeq D_{\mathfrak{f} \leftarrow, r} \otimes_{D_{r}(A)} \mathcal{M}(\mathfrak{X}) \simeq \mathcal{M}(\mathfrak{X})
$$

with $D_{r}(C)$ acting on $\mathcal{M}(\mathfrak{X})$ on the RHS by $D_{r}\left(\mathfrak{f}^{\mathrm{o}}\right)$, hence (ii) by taking the direct limit.

2.8. Assume in this subsection that $\mathfrak{f}$ is a closed immersion. Put $M=$

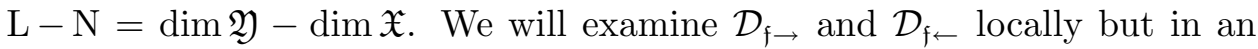
invariant manner using a Koszul complex. Thus let $\mathfrak{V}$ be an affine open of $\mathfrak{Y}$ admitting a regular system of parameters $\mathbf{z}=\left(z_{1}, \ldots, z_{\mathrm{N}+\mathrm{M}}\right)$ on $C=\mathcal{O}_{\mathfrak{Y}}(\mathfrak{V})$ such that $I=\left(z_{\mathrm{N}+1}, \ldots, z_{\mathrm{N}+\mathrm{M}}\right) \unlhd C$ with $\mathcal{O}_{\mathfrak{X}}\left(\mathfrak{f}^{-1} \mathfrak{V}\right) \simeq C / I$, and that $\left(z_{1}, \ldots, z_{\mathrm{N}}\right)$ induces a regular system of parameters on $A=C / I$. One has then a commutative diagram of short exact sequences

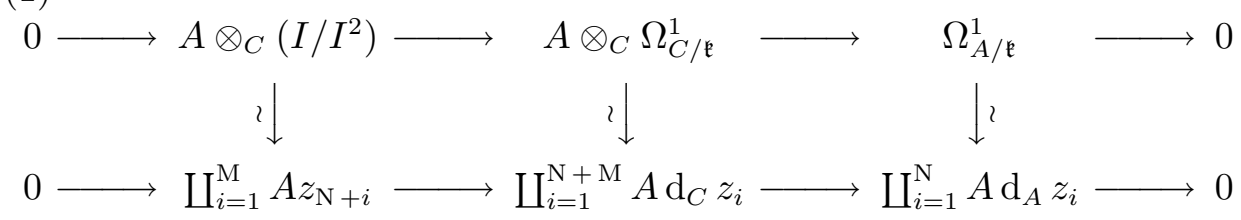

with $z_{\mathrm{N}+i} \mapsto \mathrm{d}_{C} z_{\mathrm{N}+i}$. Let $D_{\mathfrak{f} \rightarrow}=\mathcal{D}_{\mathfrak{f} \rightarrow}\left(\mathfrak{f}^{-1} \mathfrak{V}\right) \simeq A \otimes_{C} D(C)$. The left $D(A)$ module structure on $D_{\mathfrak{f} \rightarrow}$ is given by the $A$-algebra homomorphism such that

$$
\partial^{\mathbf{n}} \longmapsto 1 \otimes \partial^{(\mathbf{n}, \mathbf{0})}
$$

As $A \otimes_{C} D(C)=A \otimes_{C} \coprod_{\mathbf{n} \in \mathbb{N}^{N}, \mathbf{m} \in \mathbb{N}^{M}} C \partial^{(\mathbf{n}, \mathbf{m})} \simeq D(A) \otimes \coprod_{\mathbf{m} \in \mathbb{N}^{M}} \mathfrak{k} \partial^{(\mathbf{0}, \mathbf{m})}$,

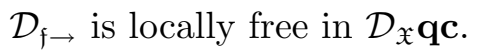

The Koszul resolution $\wedge \cdot\left(\coprod_{i=1}^{\mathrm{M}} C \mathrm{~d}_{C} z_{\mathrm{N}+i}\right) \rightarrow A$ defined by the $C$-regular sequence $\mathbf{z}=\left(z_{1}, \ldots, z_{\mathrm{N}+\mathrm{M}}\right)\left[\mathbf{M}\right.$, p. 127] induces a free resolution of $D_{\mathfrak{f} \rightarrow}$ in $\operatorname{Mod} D(C)$

$$
\left\{\wedge\left(\coprod_{i=1}^{\mathrm{M}} C \mathrm{~d}_{C} z_{\mathrm{N}+i}\right)\right\} \otimes_{C} D(C) \rightarrow A \otimes_{C} D(C) .
$$


Note that

$$
\left(\mathrm{d}_{C} z_{\mathrm{N}+i}\right)_{1 \leq i \leq \mathrm{M}} \text { is unique up to } \mathrm{GL}_{\mathrm{M}}(C) \text { in } \Omega_{C / \mathfrak{k}}^{1} \text {. }
$$

If $\mathcal{M} \in \mathcal{D}_{\mathfrak{Y}} \mathbf{q c}$ with $M=\mathcal{M}(\mathfrak{V})$,

$$
\Gamma\left(\mathfrak{f}^{-1} \mathfrak{V},\left(\mathrm{L} \mathfrak{f}^{0}\right)(\mathcal{M})\right) \simeq D_{\mathfrak{f} \rightarrow} \otimes_{D(C)}^{\mathrm{L}} M \simeq \wedge\left(\coprod_{i=1}^{\mathrm{M}} \mathfrak{k} \mathrm{d}_{C} z_{\mathrm{N}+i}\right) \otimes M
$$

that is by (5) independent of the choice of the parameters, hence these can be glued together to give a description of $\left(\mathrm{L}^{0}\right)(\mathcal{M})$.

Turning to $D_{\mathfrak{f} \leftarrow}=\mathcal{D}_{\mathfrak{f} \leftarrow}\left(\mathfrak{f}^{-1} \mathfrak{V}\right)$, that is $D_{\mathfrak{f} \rightarrow} \simeq A \otimes_{C} D(C) \simeq D(A) \otimes$ $\amalg_{\mathbf{m} \in \mathbb{N}^{\mathrm{M}}} \mathfrak{k} \partial^{(\mathbf{0}, \mathbf{m})}$ with the $D(C) \operatorname{Mod} D(A)$-structure twisted by ${ }^{*}$, one has

$$
D_{\mathfrak{f} \leftarrow} \simeq \coprod_{\mathbf{m} \in \mathbb{N}^{M}} \mathfrak{k} \partial^{(\mathbf{0}, \mathbf{m})} \otimes D(A) \quad \text { in } \operatorname{Mod} D(A) \text { via } \theta^{*} \otimes \partial \leftarrow \partial \otimes \theta,
$$

with $D(A)$ acting on the right hand side by the right regular action on $D(A)$. In particular,

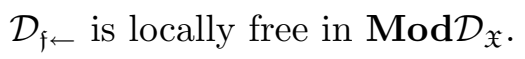

Also $D_{\mathrm{f} \leftarrow}$ admits a Koszul resolution in $D(C)$ Mod

$$
\left\{\wedge\left(\coprod_{i=1}^{\mathrm{M}} \mathfrak{k} \mathrm{d}_{C} z_{\mathrm{N}+i}\right)\right\} \otimes D(C) \rightarrow D_{\mathfrak{f} \leftarrow}
$$

As $\mathfrak{f}$ is a closed immersion, $\mathfrak{f}_{*}$ is exact on $\mathbf{A} \mathbf{b}_{\mathfrak{X}}$, hence

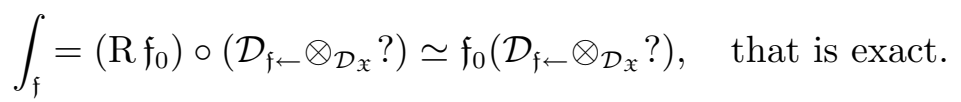

2.9. Assume $\mathfrak{f}$ is still a closed immersion. Define $\mathfrak{f}^{+}: \mathcal{D}_{\mathfrak{Y}} \mathbf{q} \mathbf{c} \rightarrow \mathcal{D}_{\mathfrak{X}} \mathbf{q c}$ via $\mathcal{M} \mapsto\left(\mathfrak{f}^{-1} \mathcal{D}_{\mathfrak{Y}}\right) \mathcal{M o d}\left(\mathcal{D}_{\mathfrak{f} \leftarrow}, \mathfrak{f}^{-1} \mathcal{M}\right)[\mathbf{H} 88,8.12 / 8.3]$. We have as in [TH, Prop. I.1.5.2]:

Proposition. Assume $\mathfrak{f}$ is a closed immersion.

(i) $[\mathbf{H 8 7}, 8.4,8.12]$ If $\mathcal{D}_{\mathfrak{Y}}^{\mathfrak{X}} \mathbf{q c}$ is the full subcategory of $\mathcal{D}_{\mathfrak{Y}} \mathbf{q c}$ consisting of the objects with support in $\mathfrak{X}$, then $\left.\mathfrak{f}^{+}\right|_{\mathcal{D}_{\mathfrak{Y}}^{x} \mathbf{q c}}$ is right adjoint to $\int_{\mathfrak{f}}^{0}$, hence left exact.

(ii) On $\mathrm{D}^{b}\left(\mathcal{D}_{\mathfrak{Y}} \mathbf{q} \mathbf{c}\right) \quad R \mathfrak{f}^{+} \simeq\left(\mathrm{L} \mathfrak{f}^{0}\right)[\operatorname{dim} \mathfrak{X}-\operatorname{dim} \mathfrak{Y}]$. 
Proof. (ii) Let $\mathcal{M} \in \mathcal{D}_{\mathfrak{Y}} \mathbf{q} \mathbf{c}$. If $\mathcal{M} \rightarrow \mathcal{I}$ is an injective resolution in $\mathcal{D}_{\mathfrak{Y}} \mathbf{q} \mathbf{c}$ and if $\mathfrak{f}^{-1} \mathcal{I} \rightarrow \mathcal{J}$ an injective resolution in $\mathfrak{f}^{-1} \mathcal{D}_{\mathfrak{Y}} \mathbf{M o d}$ [G, Th. II.7.1.1], one obtains, as $\mathfrak{f}^{-1}$ is exact, an injective resolution $\mathfrak{f}^{-1} \mathcal{M} \rightarrow \mathcal{J}$. Then in the notation of (2.7), as $\left.\mathcal{J}^{\cdot}\right|_{\mathfrak{f}^{-1} \mathfrak{V}}$ remains an injective resolution of $\left.\mathfrak{f}^{-1} \mathcal{M}\right|_{\mathfrak{f}^{-1} \mathfrak{V}}$ in $\mathfrak{f}^{-1} \mathcal{D}_{\mathfrak{V}} \operatorname{Mod}$,

$$
\begin{aligned}
\Gamma\left(\mathfrak{f}^{-1} \mathfrak{V},\left(\mathrm{Rf}^{+}\right)(\mathcal{M})\right) & \simeq\left(\mathfrak{f}^{-1} \mathcal{D}_{\mathfrak{V}}\right) \operatorname{Mod}\left(\mathcal{D}_{\mathfrak{f} \leftarrow \mid \mathfrak{f}^{-1} \mathfrak{V}},\left.\mathcal{J}^{\cdot}\right|_{\mathfrak{f}^{-1} \mathfrak{V}}\right) \\
& \simeq D(C) \operatorname{Mod}\left(\left\{\wedge\left(\coprod_{i=1}^{\mathrm{M}} \mathfrak{k} \mathrm{d}_{C} z_{\mathrm{N}+i}\right)\right\} \otimes D(C), M\right) \\
& \simeq \operatorname{Mod}_{\mathfrak{k}}\left(\wedge\left(\coprod_{i=1}^{\mathrm{M}} \mathfrak{k} \mathrm{d}_{C} z_{\mathrm{N}+i}\right), M\right) \\
& \simeq \wedge\left(\coprod_{i=1}^{\mathrm{M}} \mathfrak{k} \mathrm{d}_{C} z_{\mathrm{N}+i}\right) \otimes M[\operatorname{dim} \mathfrak{X}-\operatorname{dim} \mathfrak{Y}] \\
& \simeq \Gamma\left(\mathfrak{f}^{-1} \mathfrak{V},\left(\mathrm{L} \mathfrak{f}^{0}\right)(\mathcal{M})\right)[\operatorname{dim} \mathfrak{X}-\operatorname{dim} \mathfrak{Y}] .
\end{aligned}
$$

Taking the cohomology, the end composite isomorphism is invariant under the change of the parameters (2.8.5), hence the assertion.

2.10. Let $\mathfrak{Z}_{1} \supseteq \mathfrak{Z}_{2}$ be two closed subsets of $\mathfrak{X}$. Define $\Gamma_{\mathfrak{Z}_{i}}: \mathbf{A} \mathbf{b}_{\mathfrak{X}} \rightarrow \mathbf{A} \mathbf{b}_{\mathfrak{X}}$ by $\Gamma_{\mathfrak{Z}_{i}}(\mathcal{A})(\mathfrak{V})=\left\{a \in \mathcal{A}(\mathfrak{V}) \mid \operatorname{supp}(a) \subseteq \mathfrak{Z}_{i}\right\}, \mathcal{A} \in \mathbf{A b}_{\mathfrak{X}}, \mathfrak{V}$ open of $\mathfrak{X}, i=1,2$, and set $\Gamma_{\mathfrak{Z}_{1} / \mathfrak{Z}_{2}}=\Gamma_{\mathfrak{Z}_{1}} / \Gamma_{\mathfrak{Z}_{2}}$, i.e., $\Gamma_{\mathfrak{Z}_{1} / \mathfrak{Z}_{2}}(\mathcal{A})=\Gamma_{\mathfrak{Z}_{1}}(\mathcal{A}) / \Gamma_{\mathfrak{Z}_{2}}(\mathcal{A})$. Kempf $[\mathbf{K e}]$ defined the cohomology sheaf $\mathcal{H}_{\mathfrak{Z}_{1} / \mathcal{Z}_{2}}(\mathcal{A})$ using the Godement resolution of $\mathcal{A}$. As $\Gamma_{\mathfrak{Z}_{1} / \mathfrak{Z}_{2}}$ is exact on flasques $[\mathbf{K e}, 8.5 . \mathrm{c}, \mathrm{f}]$ and as the Godement resolution is a flasque resolution, however,

$$
\mathcal{H}_{3_{1} / 3_{2}} \simeq \mathrm{R} \Gamma_{\mathfrak{3}_{1} / 3_{2}} \text { on } \mathbf{A b}_{\mathfrak{X}} .
$$

If $\mathcal{A} \rightarrow \mathcal{F}$ is a flasque resolution, one obtains from a short exact sequence

$$
0 \rightarrow \Gamma_{\mathfrak{Z}_{2}}(\mathcal{A}) \rightarrow \Gamma_{\mathfrak{Z}_{1}}(\mathcal{A}) \rightarrow \Gamma_{\mathfrak{Z}_{1} / \mathfrak{Z}_{2}}(\mathcal{A}) \rightarrow 0
$$

an exact triangle $[\mathrm{Gr}, 4.10,5.10]$

$$
\Gamma_{\mathfrak{Z}_{2}}\left(\mathcal{F}^{\prime}\right) \rightarrow \Gamma_{\mathfrak{Z}_{1}}\left(\mathcal{F}^{\cdot}\right) \rightarrow \Gamma_{\mathfrak{Z}_{1} / \mathfrak{Z}_{2}}\left(\mathcal{F}^{\cdot}\right) \stackrel{[1]}{\longrightarrow},
$$

hence an exact triangle

$$
\mathrm{R} \Gamma_{3_{2}} \rightarrow \mathrm{R} \Gamma_{3_{1}} \rightarrow \mathrm{R} \Gamma_{3_{1} / 3_{2}} \stackrel{[1]}{\longrightarrow} \text { on } \mathrm{D}^{b}\left(\mathbf{A} \mathbf{b}_{\mathfrak{X}}\right)
$$

If $\mathfrak{Z}$ is a closed subset of $\mathfrak{X}$ and if $\mathfrak{j}: \mathfrak{X} \backslash \mathfrak{Z} \hookrightarrow \mathfrak{X}$, by [Ke, 8.2]

$$
\Gamma_{\mathfrak{X} / \mathfrak{Z}} \simeq \mathfrak{j}_{*} \mathfrak{j}^{-1} \text { on flasques, }
$$


hence

$$
\mathrm{R} \Gamma_{\mathfrak{X} / \mathfrak{Z}} \simeq \mathrm{R}\left(\mathfrak{j}_{*} \mathfrak{j}^{-1}\right) \simeq\left(\mathrm{R} \mathfrak{j}_{*}\right) \mathfrak{j}^{-1}
$$

as $\mathbf{j}^{-1}$ sends flasques to flasques, and one obtains an exact triangle

$$
\mathrm{R} \Gamma_{\mathfrak{Z}} \rightarrow \mathrm{id} \rightarrow\left(\mathrm{Rj}_{*}\right) \mathrm{j}^{-1} \stackrel{[1]}{\longrightarrow} .
$$

In particular, $R \Gamma_{\mathfrak{Z}}$ sends $\mathrm{D}^{b}\left(\mathbf{A} \mathbf{b}_{\mathfrak{X}}\right)$ to itself by Grothendieck's vanishing theorem applied to the long exact sequence induced by (5), hence also from (2) one obtains

$$
\mathrm{R} \Gamma_{\mathfrak{Z}_{1} / \mathfrak{Z}_{2}}: \mathrm{D}^{b}\left(\mathbf{A} \mathbf{b}_{\mathfrak{X}}\right) \rightarrow \mathrm{D}^{b}\left(\mathbf{A} \mathbf{b}_{\mathfrak{X}}\right)
$$

As an injective of $\mathcal{D}_{\mathfrak{X}} \mathbf{q c}$ is flasque by (1.5) and as $\int_{\mathfrak{j}} \simeq \mathrm{Rj}_{0}$ on $\mathrm{D}^{b}\left(\mathcal{D}_{\mathfrak{X}} \mathbf{q} \mathbf{c}\right)$, one obtains exact triangles on $\mathrm{D}^{b}\left(\mathcal{D}_{\mathfrak{x}} \mathbf{q} \mathbf{c}\right)$

$$
\mathrm{R} \Gamma_{\mathfrak{Z}_{2}} \rightarrow \mathrm{R} \Gamma_{\mathfrak{Z}_{1}} \rightarrow \mathrm{R} \Gamma_{\mathfrak{Z}_{1} / \mathfrak{Z}_{2}} \stackrel{[1]}{\longrightarrow}
$$

and

$$
\mathrm{R} \Gamma_{\mathfrak{Z}} \rightarrow \mathrm{id} \rightarrow \int_{\mathrm{j}} \mathrm{oj}^{-1} \stackrel{[1]}{\longrightarrow},
$$

that are compatible with the triangles (2) and (5), respectively, under the forgetful functors.

\subsection{1 .}

Lemma. If $\mathfrak{f}$ is a closed immersion, one has on $\mathrm{D}^{b}\left(\mathcal{D}_{\mathfrak{X}} \mathbf{q} \mathbf{c}\right)$

$$
\mathrm{R} \Gamma_{\mathfrak{X}} \simeq \int_{\mathfrak{f}} \circ\left(\mathrm{L} \mathfrak{f}^{0}\right)[\operatorname{dim} \mathfrak{X}-\operatorname{dim} \mathfrak{Y}]
$$

Proof. If $\mathfrak{f}_{\text {rgt }}^{+}=\operatorname{Mod}\left(\mathfrak{f}^{-1} \mathcal{D}_{\mathfrak{Y}}\right)\left(\mathcal{D}_{\mathfrak{f} \rightarrow,}, \mathfrak{f}^{-1}\right.$ ?) $: \mathbf{q c} \mathcal{D}_{\mathfrak{Y}} \rightarrow \mathbf{q c} \mathcal{D}_{\mathfrak{X}}$, one has by $[\mathrm{H} 88,8.10]$

$$
\mathfrak{f}_{\text {rgt }}^{+} \simeq \mathfrak{f}_{\text {rgt }}^{+} \circ \Gamma_{\mathfrak{X}} \quad \text { on } \mathbf{q c} \mathcal{D}_{\mathfrak{Y}} .
$$

As $\mathfrak{f}^{+} \simeq\left\{\mathfrak{f}_{\text {rgt }}^{+}\left(? \otimes_{\mathfrak{Y}} \omega_{\mathfrak{Y}}\right)\right\} \otimes_{\mathfrak{X}} \omega_{\mathfrak{X}}^{-1}$ on $\mathcal{D}_{\mathfrak{Y}} \mathbf{q} \mathbf{c}$ by $[\mathbf{H} 88,8.12]$, transferring (1) to $\mathcal{D}_{\mathfrak{Y}} \mathbf{q c}$ reads $\mathfrak{f}^{+} \simeq \mathfrak{f}^{+} \circ \Gamma_{\mathfrak{X}}$, hence by Kashiwara's equivalence $[\mathbf{H 8 8}, 8.13]$

$$
\Gamma_{\mathfrak{X}} \simeq \int_{\mathfrak{f}}^{0} \circ \mathfrak{f}^{+} \circ \Gamma_{\mathfrak{X}} \simeq \int_{\mathfrak{f}}^{0} \circ \mathfrak{f}^{+} \text {on } \mathcal{D}_{\mathfrak{Y}} \mathbf{q} \mathbf{c} .
$$


Then on $\mathrm{D}^{b}\left(\mathcal{D}_{\mathfrak{Y}} \mathbf{q} \mathbf{c}\right)$

$$
\begin{aligned}
& \mathrm{R} \Gamma_{\mathfrak{X}} \simeq \mathrm{R}\left(\int_{\mathfrak{f}}^{0} \mathfrak{f}^{+}\right) \\
& \simeq\left(\mathrm{R} \int_{\mathfrak{f}}^{0}\right) \circ \mathrm{R} \mathfrak{f}^{+} \text {as } \mathfrak{f}^{+} \text {sends injectives to injectives by }(2.8 .10,2.9 . \mathrm{i}) \\
& \simeq \int_{\mathfrak{f}} \circ\left(\mathrm{L} \mathfrak{f}^{0}\right)[\operatorname{dim} \mathfrak{X}-\operatorname{dim} \mathfrak{Y}] \text { by }(2.9 . \mathrm{ii}) .
\end{aligned}
$$

2.12. Together with (2.5) one obtains from (2.11) as in [TH, Th. I.1.8.2]:

Theorem (Base change). Given a cartesian square

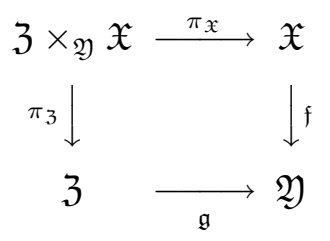

with all $\mathfrak{X}, \mathfrak{Y}, \mathfrak{Z}, \mathfrak{Z} \times_{\mathfrak{Y}} \mathfrak{X}$ smooth varieties over $\mathfrak{k}$,

$$
\left(\mathrm{L} \mathfrak{g}^{0}\right) \circ \int_{\mathfrak{f}}[\operatorname{dim} \mathfrak{Z}+\operatorname{dim} \mathfrak{X}-\operatorname{dim} \mathfrak{Y}] \simeq \int_{\pi_{\mathfrak{Z}}} \circ\left(\mathrm{L} \pi_{\mathfrak{X}}^{0}\right)\left[\operatorname{dim} \mathfrak{Z} \times_{\mathfrak{Y}} \mathfrak{X}\right] .
$$

2.13.

Example. Assume $\mathfrak{f}$ is a closed immersion, and set

$$
\mathcal{B}_{\mathfrak{X} \mid \mathfrak{Y}} \simeq \int_{\mathfrak{f}}^{0} \mathcal{O}_{\mathfrak{X}} \simeq \mathfrak{f}_{0}\left(\mathcal{D}_{\mathfrak{f} \leftarrow} \otimes_{\mathcal{D}_{\mathfrak{X}}} \mathcal{O}_{\mathfrak{X}}\right) .
$$

Locally, in the notation of (2.7), one has isomorphisms in $D(C)$ Mod

$$
\begin{aligned}
D_{\mathfrak{f} \leftarrow \otimes_{D(A)} A} & \simeq\left(A \otimes_{C} D(C)\right) \otimes_{D(A)} A \\
& \simeq\left\{(C / I) \otimes_{C} D(C)\right\} \otimes_{D(A)} A \\
& \simeq\left\{D(C) / \sum_{i=0}^{\mathrm{M}} z_{\mathrm{N}+i} D(C)\right\} \otimes_{D(A)}\left\{D(A) / \sum_{\mathbf{n} \in \mathbb{N}^{\mathrm{N}}} D(A) \partial^{\mathbf{n}}\right\} \\
& \simeq D(C) /\left\{\sum_{\mathbf{n}} \partial^{(\mathbf{n}, \mathbf{0})} D(C)+\sum_{i} z_{\mathrm{N}+i} D(C)\right\} \\
& \simeq D(C) /\left\{\sum_{\mathbf{n}} D(C) \partial^{(\mathbf{n}, \mathbf{0})}+\sum_{i} D(C) z_{\mathrm{N}+i}\right\}
\end{aligned}
$$


with the last bijection induced from $\theta \mapsto \theta^{*}$ on $D(C)$, hence the $D(C)$-action on the last term is the one induced by the left regular action.

Consider, more concretely, the case $\mathfrak{f}: \mathbb{A}_{\mathfrak{k}}^{N} \rightarrow \mathbb{A}_{\mathfrak{k}}^{N+M}$ defined by the projection $\mathfrak{k}\left[z_{1}, \ldots, z_{\mathrm{N}+\mathrm{M}}\right] \rightarrow \mathfrak{k}\left[z_{1}, \ldots, z_{\mathrm{N}}\right]$, and let $I=\left(z_{\mathrm{N}+1}, \ldots, z_{\mathrm{N}+\mathrm{M}}\right)$. By $(2.8 .6)$

$$
\Gamma\left(\mathbb{A}_{\mathfrak{k}}^{\mathrm{N}},\left(\mathrm{L} \mathfrak{f}^{0}\right)\left(\mathcal{O}_{\mathbb{A}_{\mathfrak{k}}^{\mathrm{N}}+\mathrm{M}}\right)\right) \simeq \wedge\left(\coprod_{i=1}^{\mathrm{M}} \mathfrak{k}\left[\mathbb{A}^{\mathrm{N}+\mathrm{M}}\right] \mathrm{d} z_{\mathrm{N}+i}\right),
$$

hence for each $r \in \mathbb{Z}$

$$
\Gamma\left(\mathbb{A}_{\mathfrak{k}}^{\mathrm{N}},\left(\mathrm{L}^{r} \mathfrak{f}^{0}\right)\left(\mathcal{O}_{\mathbb{A}_{\mathfrak{k}}^{\mathrm{N}}+\mathrm{M}}\right)\right) \simeq \begin{cases}\mathfrak{k}\left[\mathbb{A}^{\mathrm{N}}\right] & \text { if } r=0 \\ 0 & \text { otherwise }\end{cases}
$$

that reads in $\mathcal{D}_{\mathbb{A}_{\mathfrak{k}}^{\mathrm{N}}+\mathrm{M}} \mathbf{q} \mathbf{c}$

$$
\left(\mathrm{L}^{r} \mathfrak{f}^{0}\right)\left(\mathcal{O}_{\mathbb{A}_{\mathfrak{k}}^{\mathrm{N}}+\mathrm{M}}\right) \simeq \begin{cases}\mathcal{O}_{\mathbb{A}_{\mathfrak{k}}^{\mathrm{N}}} & \text { if } r=0 \\ 0 & \text { otherwise }\end{cases}
$$

Then in $\mathcal{D}_{\mathbb{A}_{\mathfrak{k}}^{\mathrm{N}}+\mathrm{M}} \mathbf{q} \mathbf{c}$

$$
\begin{aligned}
& \mathcal{B}_{\mathbb{A}_{\mathfrak{e}}^{N} \mid \mathbb{A}_{\mathfrak{e}}^{N}+M} \simeq \int_{\mathfrak{f}}^{0}\left(L^{0} \mathfrak{f}^{0}\right)\left(\mathcal{O}_{\mathbb{A}_{\mathfrak{e}}^{N}+M}\right) \\
& \simeq \int_{\mathfrak{f}}^{0}\left(\mathrm{~L}^{\mathrm{M}} \mathfrak{f}^{0}\right)[-\mathrm{M}]\left(\mathcal{O}_{\mathbb{A}_{\mathfrak{e}}^{\mathrm{N}}+\mathrm{M}}\right) \\
& \simeq \mathrm{H}^{\mathrm{M}}\left(\int_{\mathfrak{f}}\left(\mathrm{L} \mathfrak{f}^{0}\right)[-\mathrm{M}]\left(\mathcal{O}_{\mathbb{A}_{\mathfrak{e}}^{\mathrm{N}}+\mathrm{M}}\right)\right) \quad \text { as } \int_{\mathfrak{f}}^{0} \text { is exact and by }(2.8 .10) \\
& \simeq \mathrm{H}^{\mathrm{M}}\left(\mathrm{R} \Gamma_{\mathbb{A}_{\mathfrak{e}}^{\mathrm{N}}}\left(\mathcal{O}_{\mathbb{A}_{\mathfrak{k}}^{\mathrm{N}}+\mathrm{M}}\right)\right) \text { by }(2.11) \\
& \simeq \mathrm{R}^{\mathrm{M}} \Gamma_{\mathbb{A}_{\mathfrak{k}}^{\mathrm{N}}}\left(\mathcal{O}_{\mathbb{A}_{\mathfrak{k}}^{\mathrm{N}}+\mathrm{M}}\right) \simeq \mathcal{H}_{\mathbb{A}_{\mathfrak{k}}^{\mathrm{N}}}^{\mathrm{M}}\left(\mathcal{O}_{\mathbb{A}_{\mathfrak{k}}^{\mathrm{N}}}+\mathrm{M}\right)
\end{aligned}
$$

Likewise from (3)

$$
\mathcal{H}_{\mathbb{A}_{\mathrm{e}}^{\mathrm{N}}}^{i}\left(\mathcal{O}_{\mathbb{A}_{\mathrm{e}}^{\mathrm{N}}+\mathrm{M}}\right) \simeq \mathrm{R}^{i} \Gamma_{\mathbb{A}_{\mathrm{e}}^{\mathrm{N}}}\left(\mathcal{O}_{\mathbb{A}_{\mathrm{e}}^{\mathrm{N}}+\mathrm{M}}\right)=0 \quad \text { unless } i=\mathrm{M}
$$

$\S 3$.

In this section $\mathfrak{G}$ will denote an affine algebraic $\mathfrak{k}$-group and $\mathfrak{k}[\mathfrak{G}]$ the associated Hopf algebra with the comultiplication $\Delta_{\mathfrak{G}}$, the augmentation ideal $\mathfrak{m}_{\mathfrak{G}}$ and the antipode $\sigma_{\mathfrak{G}}$. We do not have to assume $\mathfrak{G}$ to be connected.

3.1. Let $\mathfrak{I}_{\mathfrak{G}}$ be the kernel of the multiplication $\mathfrak{k}[\mathfrak{G}] \otimes \mathfrak{k}[\mathfrak{G}] \rightarrow \mathfrak{k}[\mathfrak{G}]$, and set $P_{\mathfrak{G}}^{n}=\mathfrak{k}[\mathfrak{G}] \otimes \mathfrak{k}[\mathfrak{G}] / \mathfrak{I}_{\mathfrak{G}}^{n+1}, n \in \mathbb{N}$. We will regard $\mathfrak{k}[\mathfrak{G}] \otimes \mathfrak{k}[\mathfrak{G}]$ as $\mathfrak{k}[\mathfrak{G}]$-algebra via 
$a \mapsto a \otimes 1$, and likewise $P_{\mathfrak{G}}^{n}$. If $\mathfrak{l} \in \mathbf{S c h}_{\mathfrak{k}}(\mathfrak{G} \times \mathfrak{G}, \mathfrak{G} \times \mathfrak{G})^{\times}$via $(x, y) \mapsto(x, x y)$, then the associated $\mathfrak{k}$-algebra automorphism $\mathfrak{l}^{\circ}: \mathfrak{k}[\mathfrak{G}] \otimes \mathfrak{k}[\mathfrak{G}] \rightarrow \mathfrak{k}[\mathfrak{G}] \otimes \mathfrak{k}[\mathfrak{G}]$ is given by $a \otimes b \mapsto a \Delta_{\mathfrak{G}}(b)$. Then (cf. [Sp, 3.3.2])

$$
\mathfrak{l}\left(\mathfrak{I}_{\mathfrak{G}}\right)=\mathfrak{k}[\mathfrak{G}] \otimes \mathfrak{m}_{\mathfrak{G}},
$$

hence $\mathfrak{l}^{\circ}$ induces for each $n \in \mathbb{N}$ a $\mathfrak{k}[\mathfrak{G}]$-algebra isomorphism

$$
\mathfrak{l}_{n}^{\mathrm{o}}: P_{\mathfrak{G}}^{n} \rightarrow \mathfrak{k}[\mathfrak{G}] \otimes\left(\mathfrak{k}[\mathfrak{G}] / \mathfrak{m}_{\mathfrak{G}}^{n+1}\right)
$$

such that if $n \geq m$, there is induced a commutative diagram of $\mathfrak{k}[\mathfrak{G}]$-algebras

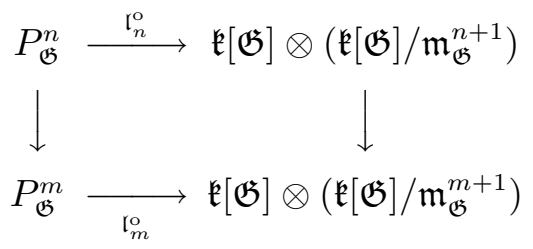

with the natural vertical homomorphisms.

3.2. For each $n \in \mathbb{N}$ let $\operatorname{Dist}_{n}(\mathfrak{G})=\left\{\mu \in \mathfrak{k}[\mathfrak{G}]^{*} \mid \mu\left(\mathfrak{m}_{\mathfrak{G}}^{n+1}\right)=0\right\}$ and $\operatorname{Dist}(\mathfrak{G})=\cup_{n \geq 0} \operatorname{Dist}_{n}(\mathfrak{G})$ the algebra of distributions of $\mathfrak{G}$. If $\mathfrak{F}_{\mathfrak{G}}: \mathfrak{G} \rightarrow$ $\mathfrak{G}^{(r)}$ is the $r$-th Frobenius morphism on $\mathfrak{G}$, let $\mathfrak{G}_{r}=\operatorname{ker}\left(\mathfrak{F}_{\mathfrak{G}}^{r}\right)$ with $\mathfrak{k}\left[\mathfrak{G}_{r}\right]=$ $\mathfrak{k}[\mathfrak{G}] /\left(a^{p^{r}} \mid a \in \mathfrak{m}_{\mathfrak{G}}\right)$. If $\Lambda_{n}=\mathfrak{k}[\mathfrak{G}] \operatorname{Mod}\left(\mathfrak{l}_{n}^{\circ}, \mathfrak{k}[\mathfrak{G}]\right)$ one obtains a commutative diagram of $\mathfrak{k}[\mathfrak{G}]$-modules

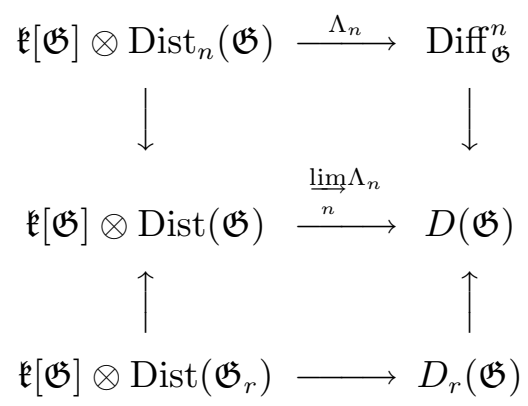

with the vertical maps being inclusions and the bijective horizontal maps given by $a \otimes \mu \mapsto(a \otimes \mu) \circ \Delta_{\mathfrak{G}}$. Moreover, if one defines a $\mathfrak{G}$-action on $D(\mathfrak{G})$ by $(x \delta)(a)=\delta(a x) x^{-1}$ with $a x=a(x ?), x \in \mathfrak{G}$ and $a \in \mathfrak{k}[\mathfrak{G}]$, and if $D^{\mathrm{lft}}(\mathfrak{G})=\{\delta \in D(\mathfrak{G}) \mid x \delta=\delta \forall x \in \mathfrak{G}\}$, then the middle horizontal bijection in (1) induces a $\mathfrak{k}$-algebra isomorphsm (cf. [DG, II.4.6.5])

$$
\operatorname{Dist}(\mathfrak{G}) \rightarrow D^{\mathrm{lft}}(\mathfrak{G})
$$

For our purposes, however, it is more convenient to work with $\mathfrak{r} \in \mathbf{S c h}_{\mathfrak{k}}(\mathfrak{G} \times$ $\mathfrak{G}, \mathfrak{G} \times \mathfrak{G})^{\times}$via $(x, y) \mapsto(x, y x)$ in place of $\mathfrak{l}$ in $(3.1)$ to obtain: 


\section{3 .}

Proposition. For each $r \in \mathbb{N}$ there is a commutative diagram of $\mathfrak{k}[\mathfrak{G}]$ modules

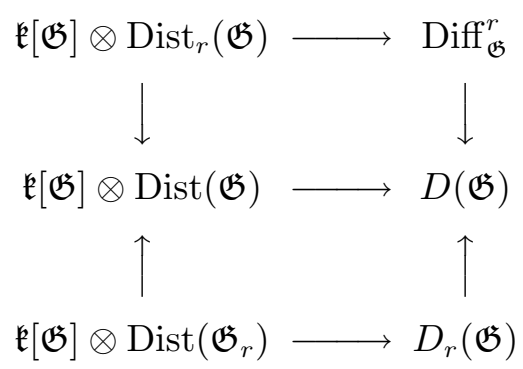

with the vertical arrows being inclusions and the horizontal ones bijective given by $a \otimes \mu \mapsto a(\mu \otimes \mathfrak{k}[\mathfrak{G}]) \circ \Delta_{\mathfrak{G}}$. If $D^{\mathrm{rgt}}(\mathfrak{G})=\{\delta \in D(\mathfrak{G}) \mid \delta x=\delta \forall x \in$ $\mathfrak{G}\}$ with $(\delta x)(a)=x^{-1} \delta(x a)$ and $x a=a(? x) \forall a \in \mathfrak{k}[\mathfrak{G}]$, then the middle horizontal bijection induces a $\mathfrak{k}$-algebra isomorphism $\operatorname{Dist}(\mathfrak{G})^{\mathrm{op}} \rightarrow D^{\mathrm{rgt}}(\mathfrak{G})$.

3.4. Let $\mathfrak{a} \in \mathbf{S c h}_{\mathfrak{k}}(\mathfrak{G} \times \mathfrak{X}, \mathfrak{X})$ be a group action of $\mathfrak{G}$ on a smooth variety $\mathfrak{X}$. We will call such a triple $(\mathfrak{G}, \mathfrak{X}, \mathfrak{a})$ a $\mathfrak{G}$-variety, and denote the category of $\mathfrak{G}$-varieties by $\mathbf{S c h}_{\mathfrak{k}}^{\mathfrak{G}}$. A $\mathfrak{G}$-equivariant $\mathcal{O}_{\mathfrak{X}}$-module is a pair $(\mathcal{M}, \phi)$ of $\mathcal{M} \in \mathbf{q c}_{\mathfrak{X}}$ and $\phi \in \operatorname{Mod}_{\mathfrak{G} \times \mathfrak{X}}\left(\mathfrak{a}^{*} \mathcal{M}, \mathfrak{p}_{\mathfrak{X}}^{*} \mathcal{M}\right)^{\times}$such that the diagram

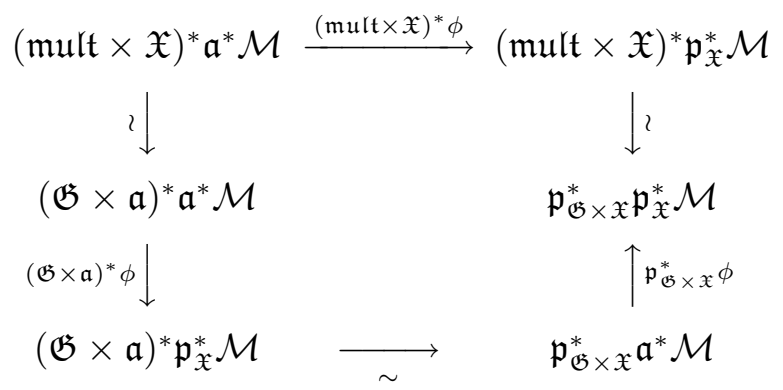

commutes, where the $\mathfrak{p}$ 's are the projections and ${ }^{\times}$indicates that $\phi$ is invertible. A morphism $f:\left(\mathcal{M}, \phi_{\mathcal{M}}\right) \rightarrow\left(\mathcal{N}, \phi_{\mathcal{N}}\right)$ of $\mathfrak{G}$-equivariant $\mathcal{O}_{\mathfrak{X}}$-modules is a morphism $f: \mathcal{M} \rightarrow \mathcal{N}$ of $\mathcal{O}_{\mathfrak{X}}$-modules such that $\phi_{\mathcal{N}} \circ \mathfrak{a}^{*} f=\mathfrak{p}_{\mathfrak{X}}^{*} f \circ \phi_{\mathcal{M}}$. We will denote the category of $\mathfrak{G}$-equivariant $\mathcal{O}_{\mathfrak{X}}$-modules by $(\mathfrak{G}, \mathfrak{X}) \mathbf{q}$.

If $(\mathcal{M}, \phi) \in(\mathfrak{G}, \mathfrak{X}) \mathbf{q} \mathbf{c}$ and if $\mathfrak{i}_{\mathfrak{X}}: \mathfrak{X} \rightarrow \mathfrak{G} \times \mathfrak{X}$ via $x \mapsto(e, x)$, one obtains a commutative diagram

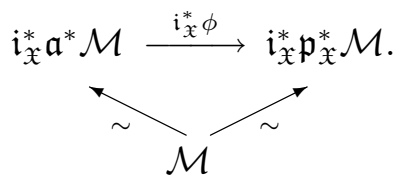


If $\mathfrak{a}^{\prime} \in \mathbf{S c h}_{\mathfrak{k}}(\mathfrak{G} \times \mathfrak{X}, \mathfrak{G} \times \mathfrak{X})^{\times}$such that $(g, x) \mapsto(g, g x)$, then

$$
\mathfrak{a}=\mathfrak{p}_{\mathfrak{X}} \circ \mathfrak{a}^{\prime} .
$$

As $\mathfrak{p}_{\mathfrak{X}}$ and $\mathfrak{p}_{\mathfrak{G} \times \mathfrak{X}}$ are both affine and flat and as $\mathfrak{a}^{\prime}$ is invertible,

$$
\mathfrak{a}, \mathfrak{m u l t} \times \mathfrak{X} \text {, and } \mathfrak{G} \times \mathfrak{a} \text { are all affine and flat. }
$$

In particular,

(5) $\quad$ all $\mathfrak{p}_{\mathfrak{X}}^{*}, \mathfrak{a}^{*}$, and $\mathfrak{p}_{\mathfrak{G} \times \mathfrak{X}}^{*},(\mathfrak{m u l t} \times \mathfrak{X})^{*},(\mathfrak{G} \times \mathfrak{a})^{*}$ are exact

on $\operatorname{Mod}_{\mathfrak{X}}$ and $\operatorname{Mod}_{\mathfrak{G} \times \mathfrak{X}}$, respectively.

Then for any $f \in(\mathfrak{G}, \mathfrak{X}) \mathbf{q c}$, both ker $f$ and coker $f$ belong to $(\mathfrak{G}, \mathfrak{X}) \mathbf{q c}$, hence

$$
(\mathfrak{G}, \mathfrak{X}) \mathbf{q c} \text { is an abelian category. }
$$

If we define the category $\mathrm{D}_{\mathfrak{G}}^{b}\left(\mathbf{q c}_{\mathfrak{X}}\right)$ to consist of all pairs $(\mathcal{M}, \phi)$ of $\mathcal{M} \in$ $\mathrm{D}^{b}\left(\mathbf{q c}_{X}\right)$ and $\phi \in \mathrm{D}^{b}\left(\mathbf{q c}_{\mathfrak{G} \times \mathfrak{X}}\right)\left(\mathfrak{a}^{*} \mathcal{M} \cdot \mathfrak{p}_{\mathfrak{X}}^{*} \mathcal{M}\right)^{\times}$such that the diagram (1) commutes in $\mathrm{D}^{b}\left(\mathbf{q c}_{\mathfrak{G} \times \mathfrak{X}}\right)$ with $\mathcal{M}$ replacing $\mathcal{M}$. If $(\mathcal{M}, \phi) \in \mathrm{D}_{\mathfrak{G}}^{b}\left(\mathbf{q c}_{X}\right)$, each $\mathrm{H}^{i}\left(\mathcal{M}^{\cdot}\right), i \in \mathbb{Z}$, carries by (6) a structure of $(\mathfrak{G}, \mathfrak{X}) \mathbf{q c}$. Hence

$$
\begin{aligned}
& \mathrm{D}^{b}((\mathfrak{G}, \mathfrak{X}) \mathbf{q c}) \text { is equivalent to } \mathrm{D}_{\mathfrak{G}}^{b}\left(\mathbf{q} \mathbf{c}_{\mathfrak{X}}\right) \text { under the forgetful functor } \\
& \text { with quasi-inverse }(\mathcal{M}, \phi) \mapsto\left(\mathrm{H}^{\cdot}\left(\mathcal{M}^{\cdot}\right), \mathrm{H}^{\cdot}(\phi)\right) .
\end{aligned}
$$

3.5. Let $\mathfrak{f} \in \operatorname{Sch}_{\mathfrak{k}}(\mathfrak{X}, \mathfrak{Y}), \mathcal{M} \in \operatorname{Mod}_{\mathfrak{X}}, \mathcal{N} \in \operatorname{Mod}_{\mathfrak{Y}}$, and $\delta \in \operatorname{Mod}_{\mathfrak{k}}\left(\mathcal{N}, \mathfrak{f}_{*} \mathcal{M}\right)$. Slightly extending the definition of [DG, II.4.5.1] we say a pair $(\mathfrak{f}, \delta)$ is a $\mathfrak{k}$ deviation of order $\leq n, n \in \mathbb{N}$, iff

$$
\left\{\operatorname{ad}\left(a_{0}\right) \ldots \operatorname{ad}\left(a_{n}\right)\right\} \delta(\mathfrak{U})=0 \quad \forall a_{i} \in \mathcal{O}_{\mathfrak{Y}}(\mathfrak{U}), \mathfrak{U} \text { open of } \mathfrak{Y}
$$

where $\operatorname{ad}\left(a_{i}\right) \delta(\mathfrak{U})=a_{i} \delta(\mathfrak{U})-\delta(\mathfrak{U}) a_{i}$. In particular (cf. [DG, II.4.5.4, 5], [EGAIV, 16.8.3]),

(1) the set of $\mathfrak{k}$-deviations of order $\leq n$ for $\mathfrak{f}=\operatorname{id}_{\mathfrak{X}}$ and $\mathcal{N}=\mathcal{M}$ is just

$$
\operatorname{Diff}_{\mathfrak{X}}^{n}(\mathcal{M})=\operatorname{Diff}_{\mathfrak{X} / \mathfrak{k}}^{n}(\mathcal{M}, \mathcal{M}) .
$$

Also if $\varepsilon_{\mathfrak{G}}: \mathfrak{e} \rightarrow \mathfrak{G}$ is the unit section, then [DG, II.4.6.2]

(2) $\operatorname{Dist}_{n}(\mathfrak{G})$ may be identified with the set of all deviations $\left(\varepsilon_{\mathfrak{G}}, \mu\right)$

$$
\text { with } \mu \in \operatorname{Mod}_{\mathfrak{k}}\left(\mathcal{O}_{\mathfrak{G}},\left(\varepsilon_{\mathfrak{G}}\right)_{*} \mathcal{O}_{\mathfrak{e}}\right) \text { of order } \leq n \text {. }
$$


Now let $(\mathcal{M}, \phi) \in(\mathfrak{G}, \mathfrak{X}) \mathbf{q c}$ and $\tilde{\phi} \in \operatorname{Mod}_{\mathfrak{X}}\left(\mathcal{M}, \mathfrak{a}_{*} \mathfrak{p}_{\mathfrak{X}}^{*} \mathcal{M}\right)$ the adjoint of $\phi$. Then $(\mathfrak{a}, \tilde{\phi})$ is a deviation of order 0 . Let $\mathfrak{i}_{\mathfrak{X}}^{\prime} \in \mathbf{S c h}_{\mathfrak{k}}(\mathfrak{X}, \mathfrak{e} \times \mathfrak{X})^{\times}$induced by $\mathfrak{i}_{\mathfrak{X}}$ and let $\mathcal{M}^{\prime}=\left(\mathfrak{i}_{\mathfrak{X}}^{\prime}\right)_{*} \mathcal{M}$. Then $\left(\mathfrak{i}_{\mathfrak{X}}^{\prime}, \operatorname{id}_{\mathcal{M}^{\prime}}: \mathcal{M}^{\prime} \rightarrow\left(\mathfrak{i}_{\mathfrak{X}}^{\prime}\right)_{*} \mathcal{M}\right)$ is also a deviation of order 0 . If $\mu \in \operatorname{Dist}_{n}(\mathfrak{G})$, define a deviation $\left(\varepsilon_{\mathfrak{G}} \times \mathfrak{X}: \mathfrak{e} \times \mathfrak{X} \rightarrow\right.$ $\left.\mathfrak{G} \times \mathfrak{X}, \mu \otimes \mathcal{M}: \mathfrak{p}_{\mathfrak{X}}^{*} \mathcal{M} \rightarrow\left(\varepsilon_{\mathfrak{G}} \times \mathfrak{X}\right)_{*} \mathcal{M}^{\prime}\right)$ of order 0 [DG, II.4.5.11] by the commutative diagram

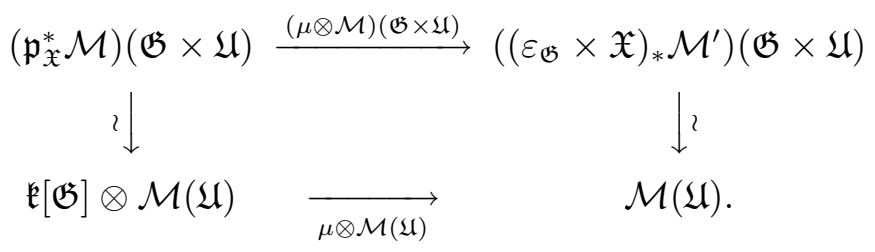

Then the composite deviation

$$
\left(\operatorname{id}_{\mathfrak{X}}=\mathfrak{a} \circ\left(\varepsilon_{\mathfrak{G}} \times \mathfrak{X}\right) \circ \mathfrak{i}_{\mathfrak{X}}^{\prime}, \mathfrak{a}_{*}\left(\varepsilon_{\mathfrak{G}} \times \mathfrak{X}\right)_{*}\left(\operatorname{id}_{\mathcal{M}^{\prime}}\right) \circ \mathfrak{a}_{*}(\mu \otimes \mathcal{M}) \circ \tilde{\phi}: \mathcal{M} \rightarrow \mathcal{M}\right)
$$

is of order $\leq n$ [DG, II.4.6.3]. On the other hand, for $n \in \mathbb{N}$ let $\mathfrak{V}_{n}(\mathfrak{e})=$ $\mathfrak{S p}_{\mathfrak{k}}\left(\mathfrak{k}[\mathfrak{G}] / \mathfrak{m}_{\mathfrak{G}}^{n+1}\right), \varepsilon_{n}: \mathfrak{V}_{n}(\mathfrak{e}) \hookrightarrow \mathfrak{G}, \mathfrak{a}_{n}=\mathfrak{a} \circ\left(\varepsilon_{n} \times \mathfrak{X}\right): \mathfrak{V}_{n}(\mathfrak{e}) \times \mathfrak{X} \rightarrow \mathfrak{X}$, $\mathfrak{p}_{\mathfrak{X}, n}=\mathfrak{p}_{\mathfrak{X}} \circ\left(\varepsilon_{n} \times \mathfrak{X}\right): \mathfrak{V}_{n}(\mathfrak{e}) \times \mathfrak{X} \rightarrow \mathfrak{X}$ the projection, $\phi_{n}=\left(\varepsilon_{n} \times \mathfrak{X}\right)^{*} \phi:$ $\mathfrak{a}_{n}^{*} \mathcal{M} \rightarrow \mathfrak{p}_{\mathfrak{X}, n}^{*} \mathcal{M}$, and $\tilde{\phi}_{n}: \mathcal{M} \rightarrow\left(\mathfrak{a}_{n}\right)_{*} \mathfrak{p}_{\mathfrak{X}, n}^{*} \mathcal{M}$ the adjoint of $\phi_{n}$. Define $\phi^{\prime}: \operatorname{Dist}(\mathfrak{G}) \rightarrow \operatorname{Mod}_{\mathfrak{k}}(\mathcal{M}, \mathcal{M})$ by the commutative diagram

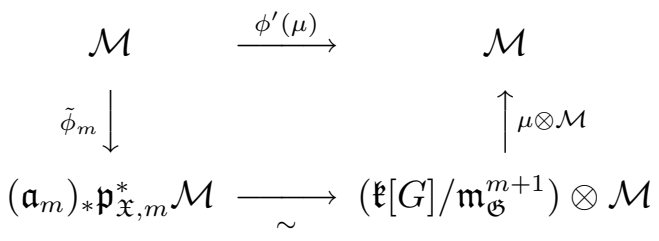

for $\mu \in \operatorname{Dist}_{n}(\mathfrak{G})$ and $m \geq n$, that is independent of the choice of $m$. If $\varepsilon_{0, n}: \mathfrak{e} \rightarrow \mathfrak{V}_{n}(\mathfrak{e})$ is the inclusion and if we define a deviation $\left(\varepsilon_{0, n} \times \mathfrak{X}, \mu \otimes \mathcal{M}:\right.$ $\left.\mathfrak{p}_{\mathfrak{X}}^{*} \mathcal{M} \rightarrow\left(\varepsilon_{0, n} \times \mathfrak{X}\right)_{*} \mathcal{M}^{\prime}\right)$ as in $(3)$, then

$$
\phi^{\prime}(\mu)=\left(\mathfrak{a}_{n}\right)_{*}(\mu \otimes \mathcal{M}) \circ \tilde{\phi}_{n}
$$

under the identification of $\left(\mathfrak{a}_{n}\right)_{*}\left(\varepsilon_{0, n} \times \mathfrak{X}\right)_{*} \mathcal{M}^{\prime}$ with $\mathcal{M}$, and the composite deviation $\left(\operatorname{id}_{\mathfrak{X}}=\mathfrak{a}_{n} \circ\left(\varepsilon_{0, n} \times \mathfrak{X}\right) \circ \mathfrak{i}_{\mathfrak{X}}^{\prime}, \phi^{\prime}(\mu)\right)$ coincides with the deviation (4), hence $\phi^{\prime}(\mu) \in \operatorname{Diff}_{\mathfrak{X}}^{n}(\mathcal{M})$.

3.6.

Proposition. If $(\mathcal{M}, \phi) \in(\mathfrak{G}, \mathfrak{X}) \mathbf{q c}$, the $\mathfrak{k}$-linear map $\phi^{\prime}$ of (3.5.2) induces $a \mathfrak{k}$-algebra homomorphism $\operatorname{Dist}(\mathfrak{G})^{\mathrm{op}} \rightarrow \operatorname{Diff}_{\mathfrak{X}}(\mathcal{M})$, still denoted $\phi^{\prime}$, under which

$$
\operatorname{Dist}_{r}(\mathfrak{G})^{\text {op }} \rightarrow \operatorname{Diff}_{\mathfrak{X}}^{r}(\mathcal{M})
$$


and

$$
\operatorname{Dist}\left(\mathfrak{G}_{r}\right)^{\text {op }} \rightarrow D_{r}(\mathcal{M})=\operatorname{Mod}_{\mathcal{O}_{\mathfrak{X}}^{(r)}}(\mathcal{M}, \mathcal{M}) \quad \forall r \in \mathbb{N}
$$

Proof. The assertion that $\phi^{\prime}$ induces a $\mathfrak{k}$-algebra homomorphism follows from the commutative diagrams $(3.4 .1,2)$. To see the last assertion, let $\mu \in$ $\operatorname{Dist}\left(\mathfrak{G}_{r}\right), M=\mathcal{M}(\mathfrak{U}), \mathfrak{U}$ an affine open of $\mathfrak{X}$, and $C=\mathcal{O}_{\mathfrak{X}}(\mathfrak{U})$. We must show

$$
\phi^{\prime}(\mu)(\mathfrak{U})\left(c^{p^{r}} m\right)=c^{p^{r}} \phi^{\prime}(\mu)(\mathfrak{U})(m) \quad \forall c \in C \text { and } m \in M .
$$

As $\mathfrak{G}_{r}$ is infinitesimal, the action $\mathfrak{a}$ induces an action $\mathfrak{a}_{r}: \mathfrak{G}_{r} \times \mathfrak{U} \rightarrow \mathfrak{U}$. If $\mathfrak{i}_{r}: \mathfrak{G}_{r} \rightarrow \mathfrak{G}$ and $\mathfrak{j}: \mathfrak{U} \rightarrow \mathfrak{X}$ are two inclusions and if $\psi_{r}$ is the adjoint of $\left(\mathfrak{i}_{r} \times \mathfrak{j}\right)^{*} \phi$, then

$$
\phi^{\prime}(\mu)(\mathfrak{U})=(\mu \otimes M) \circ \psi_{r}(\mathfrak{U}) .
$$

Also $\left(\varepsilon_{\mathfrak{G}_{r}} \times \mathfrak{U}\right)^{\circ} \circ \mathfrak{a}_{r}^{o}=\mathrm{id}_{\mathfrak{k}[\mathfrak{U}]}$ with $\varepsilon_{\mathfrak{G}_{r}}$ the unit section of $\mathfrak{G}_{r}$, hence one can write

$$
\mathfrak{a}_{r}^{\mathrm{o}}(c)=1 \otimes c+\sum_{i} a_{i} \otimes c_{i}, \quad a_{i} \in \mathfrak{m}_{\mathfrak{G}} \mathfrak{k}\left[\mathfrak{G}_{r}\right], c_{i} \in C .
$$

If $\psi_{r}(\mathfrak{U})(m)=\sum_{j} b_{j} \otimes m_{j}, b_{j} \in \mathfrak{k}\left[\mathfrak{G}_{r}\right], m_{j} \in M$, then

$$
\psi_{r}(\mathfrak{U})\left(c^{p^{r}} m\right)=\left(\left(\mathfrak{i}_{r} \times \mathfrak{j}\right)^{*} \phi\right)\left(\mathfrak{G}_{r} \times \mathfrak{U}\right)\left(\mathfrak{a}_{r}^{\mathrm{o}}\left(c^{p^{r}}\right) \otimes m\right)=\sum_{j} b_{j} \otimes c^{p^{r}} m_{j}
$$

as $a_{i}^{p^{r}}=0$, hence $\phi^{\prime}(\mu)(\mathfrak{U})\left(c^{p^{r}} m\right)=\sum_{j} \mu\left(b_{j}\right) c^{p^{r}} m_{j}=c^{p^{r}} \phi^{\prime}(\mu)(\mathfrak{U})(m)$.

3.7. Conversely,

Lemma (infinitesimal test). Assume $\mathcal{M} \in \mathbf{q c}_{\mathfrak{X}}$ is of finite type or locally free. Let $\phi \in \operatorname{Mod}_{\mathfrak{X}}\left(\mathfrak{a}^{*} \mathcal{M}, \mathfrak{p}_{\mathfrak{X}}^{*} \mathcal{M}\right)^{\times}$. If $\phi^{\prime}$ induces a $\mathfrak{k}$-algebra homomorphism $\operatorname{Dist}(\mathfrak{G})^{\mathrm{op}} \rightarrow \operatorname{Mod}_{\mathfrak{k}}\left(\mathcal{M}_{x}, \mathcal{M}_{x}\right)$ at each $x \in \mathfrak{X}(\mathfrak{k})$, i.e., each $\mathcal{M}_{x}$ is a $\mathfrak{G}_{r}^{\text {op }}$ module for all $r \in \mathbb{N}$ compatibly, then the diagram (3.4.1) commutes, hence defines a structure of $(\mathfrak{G}, \mathfrak{X}) \mathbf{q c}$ on $\mathcal{M}$. If $\mathfrak{G}$ is transitive on $\mathfrak{X}$, one has only to test the criterion at arbitrary one point of $\mathfrak{X}(\mathfrak{k})$.

Proof. We will show that the diagram (3.4.1) commutes at each point $\left(g_{1}, g_{2}, x\right) \in \mathfrak{G}(\mathfrak{k}) \times \mathfrak{G}(\mathfrak{k}) \times \mathfrak{X}(\mathfrak{k}):$

$$
\begin{aligned}
&\left(\mathfrak{p}_{2}^{*} \phi\right)_{\left(g_{1}, g_{2}, x\right)}=\left(\mathfrak{p}_{3}^{*} \phi\right)_{\left(g_{1}, g_{2}, x\right)} \circ\left(\mathfrak{p}_{1}^{*} \phi\right)_{\left(g_{1}, g_{2}, x\right)}: \\
& \mathcal{O}_{\mathfrak{G} \times \mathfrak{G} \times \mathfrak{X},\left(g_{1}, g_{2}, x\right)} \otimes_{\mathcal{O}_{\mathfrak{X}, g_{1} g_{2} x}} \mathcal{M}_{g_{1} g_{2} x} \rightarrow \mathcal{O}_{\mathfrak{G} \times \mathfrak{G} \times \mathfrak{X},\left(g_{1}, g_{2}, x\right)} \otimes_{\mathcal{O}_{\mathfrak{X}, x}} \mathcal{M}_{x},
\end{aligned}
$$


where $\mathfrak{p}_{1}=\mathfrak{G} \times \mathfrak{a}, \mathfrak{p}_{2}=\mathfrak{m u l t} \times \mathfrak{X}$, and $\mathfrak{p}_{3}=\mathfrak{p}_{\mathfrak{G} \times \mathfrak{X}}$. For that it is enough by Krull's intersection theorem [M, Th. 8.9, 10] to show the equality under the natural surjection

$\mathcal{O}_{\mathfrak{G} \times \mathfrak{G} \times \mathfrak{X},\left(g_{1}, g_{2}, x\right)} \otimes_{\mathcal{O}_{\mathfrak{X}, x}} \mathcal{M}_{x} \rightarrow\left\{\mathcal{O}_{\mathfrak{G} \times \mathfrak{G} \times \mathfrak{X},\left(g_{1}, g_{2}, x\right)} / \mathfrak{m}_{\left(g_{1}, g_{2}, x\right)}^{r+1}\right\} \otimes_{\mathcal{O}_{\mathfrak{X}, x}} \mathcal{M}_{x} \forall r \in \mathbb{N}$

where $\mathfrak{m}_{\left(g_{1}, g_{2}, x\right)}^{r+1}$ is the maximal ideal of $\mathcal{O}_{\mathfrak{G} \times \mathfrak{G} \times \mathfrak{X},\left(g_{1}, g_{2}, x\right)}$. For simplicity put $A=\mathfrak{k}[\mathfrak{G}]$, and let $\mathfrak{U}$ be an affine open neighbourhood of $x$ in $\mathfrak{X}, C=\mathcal{O}_{\mathfrak{X}}(\mathfrak{U})$, $\mathfrak{V}_{r}\left(g_{i}\right)=\mathfrak{S p}_{\mathfrak{k}}\left(A / \mathfrak{m}_{i}^{r+1}\right)$ with $\mathfrak{m}_{i}=\operatorname{ker} g_{i} \in \operatorname{Max}(A)$, and $\overline{\mathfrak{m}}$ the maximal ideal of $\mathcal{O}_{\mathfrak{V}_{r}\left(g_{1}\right) \times \mathfrak{V}_{r}\left(g_{2}\right) \times \mathfrak{U},\left(g_{1}, g_{2}, x\right)}$. Then

$$
\mathcal{O}_{\mathfrak{G} \times \mathfrak{G} \times \mathfrak{X},\left(g_{1}, g_{2}, x\right)} / \mathfrak{m}_{\left(g_{1}, g_{2}, x\right)}^{r+1} \simeq \mathcal{O}_{\mathfrak{V}_{r}\left(g_{1}\right) \times \mathfrak{V}_{r}\left(g_{2}\right) \times \mathfrak{U},\left(g_{1}, g_{2}, x\right)} / \overline{\mathfrak{m}}^{r+1} .
$$

Hence, if $\mathfrak{i}_{r}^{g_{i}}: \mathfrak{V}_{r}\left(g_{i}\right) \rightarrow \mathfrak{G}$ and $\mathfrak{j}: \mathfrak{U} \rightarrow \mathfrak{X}$ are inclusions, the equality (1) will follow from

(2) $\left\{\left(\mathfrak{i}_{r}^{g_{1}} \times \mathfrak{i}_{r}^{g_{2}} \times \mathfrak{j}\right)^{*} \mathfrak{p}_{2}^{*} \phi\right\}_{\left(g_{1}, g_{2}, x\right)}=\left\{\left(\mathfrak{i}_{r}^{g_{1}} \times \mathfrak{i}_{r}^{g_{2}} \times \mathfrak{j}\right)^{*}\left(\left(\mathfrak{p}_{3}^{*} \phi\right) \circ\left(\mathfrak{p}_{1}^{*} \phi\right)\right)\right\}_{\left(g_{1}, g_{2}, x\right)}$.

But $\mathfrak{i}_{r}^{g_{i}}=\mathfrak{i}_{r} \circ g_{i}^{-1}$ if $\mathfrak{i}_{r}=\mathfrak{i}_{r}^{e}$ and if $g_{i}: \mathfrak{V}_{r}(e) \rightarrow \mathfrak{V}_{r}\left(g_{i}\right)$ is the translation by $g_{i}$. As $\mathfrak{i}_{r}^{g_{1}} \times \mathfrak{i}_{r}^{g_{2}} \times \mathfrak{j}=\left(\mathfrak{i}_{r} \times \mathfrak{i}_{r} \times \mathfrak{j}\right) \circ\left(g_{1}^{-1} \times g_{2}^{-1} \times \mathfrak{U}\right)$ and as $g_{1}^{-1} \times g_{2}^{-1} \times \mathfrak{U}$ : $\mathfrak{V}_{r}\left(g_{1}\right) \times \mathfrak{V}_{r}\left(g_{2}\right) \times \mathfrak{U} \rightarrow \mathfrak{V}_{r}(e) \times \mathfrak{V}_{r}(e) \times \mathfrak{U}$ is invertible, we may assume $g_{1}=g_{2}=e$ in $(2)$. Let $\mathfrak{a}_{2 r}=\mathfrak{a} \circ\left(\mathfrak{i}_{2 r} \times \mathfrak{j}\right), \mathfrak{p}_{\mathfrak{X}, 2 r}=\mathfrak{p}_{\mathfrak{X}} \circ \mathfrak{i}_{2 r}$, and $\phi_{2 r}=\left(\mathfrak{i}_{2 r} \times \mathfrak{j}\right)^{*} \phi$. If we define $\mathfrak{p}_{i, r}, 1 \leq i \leq 3$, by the commutative diagrams

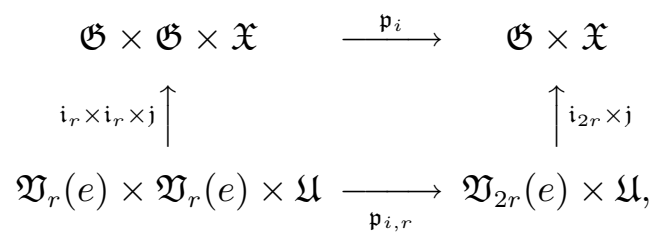

then the equality (2) reads

$$
\begin{aligned}
\left(\mathfrak{p}_{2, r}^{*} \phi_{2 r}\right)_{(e, e, x)}=\left(\mathfrak{p}_{3, r}^{*} \phi_{2 r}\right)_{(e, e, x)} \circ & \left(\mathfrak{p}_{1, r}^{*} \phi_{2 r}\right)_{(e, e, x)}: \\
& \left(\mathfrak{p}_{2, r}^{*} \mathfrak{a}_{2 r}^{*} \mathcal{M}\right)_{(e, e, x)} \rightarrow\left(\mathfrak{p}_{2, r}^{*} \mathfrak{p}_{\mathfrak{X}, 2 r}^{*} \mathcal{M}\right)_{(e, e, x)},
\end{aligned}
$$

that in turn will follow from

$$
\begin{aligned}
\left(\mathfrak{p}_{2, r}^{*} \phi_{2 r}\right) & \left(\mathfrak{V}_{r}(e) \times \mathfrak{V}_{r}(e) \times \mathfrak{U}\right) \\
= & \left(\mathfrak{p}_{3, r}^{*} \phi_{2 r}\right)\left(\mathfrak{V}_{r}(e) \times \mathfrak{V}_{r}(e) \times \mathfrak{U}\right) \circ\left(\mathfrak{p}_{1, r}^{*} \phi_{2 r}\right)\left(\mathfrak{V}_{r}(e) \times \mathfrak{V}_{r}(e) \times \mathfrak{U}\right) .
\end{aligned}
$$

As both sides are $\mathfrak{k}\left[\mathfrak{V}_{r}(e) \times \mathfrak{V}_{r}(e) \times \mathfrak{U}\right]$-linear, we have only to verify (4) after composing with the natural homomorphism

$$
\mathcal{M}(\mathfrak{U}) \rightarrow\left(\mathfrak{p}_{2, r}^{*} \mathfrak{a}_{2 r}^{*} \mathcal{M}\right)\left(\mathfrak{V}_{r}(e) \times \mathfrak{V}_{r}(e) \times \mathfrak{U}\right) .
$$


Let $\psi_{r}: \mathcal{M} \rightarrow\left(\mathfrak{a}_{r}\right)_{*} \mathfrak{p}_{\mathfrak{X}, r}^{*} \mathcal{M}$ be the adjoint of $\phi_{r}, M=\mathcal{M}(\mathfrak{U}), \Delta_{M, r}=\psi_{r}(\mathfrak{U})$ : $M \rightarrow\left(A / \mathfrak{m}_{\mathfrak{G}}^{r+1}\right) \otimes M$, and define likewise $\Delta_{M, 2 r}$ from $\phi_{2 r}$. If $\mathfrak{i}_{r, 2 r}: \mathfrak{V}_{r}(e) \rightarrow$ $\mathfrak{V}_{2 r}(e)$ is the inclusion and if $\Delta_{\mathfrak{G}, r}: A / \mathfrak{m}_{\mathfrak{G}}^{2 r+1} \rightarrow\left(A / \mathfrak{m}_{\mathfrak{G}}^{r+1}\right) \otimes\left(A / \mathfrak{m}_{\mathfrak{G}}^{r+1}\right)$ is the $\mathfrak{k}$-algebra homomorphism induced by the multiplication $\mathfrak{V}_{r}(e) \times \mathfrak{V}_{r}(e) \rightarrow$ $\mathfrak{V}_{2 r}(e)$, the LHS (resp. RHS) of (4) composed with (5) reads $\left(\mathfrak{i}_{r, 2 r}^{\circ} \otimes \Delta_{M, r}\right) \circ$ $\Delta_{M, 2 r}$ (resp. $\left.\left(\Delta_{\mathfrak{G}, r} \otimes M\right) \circ \Delta_{M, 2 r}\right)$. But from the hypothesis one has

$\left\{\left(\mathfrak{i}_{r, 2 r}^{\circ} \otimes \Delta_{M, r}\right) \circ \Delta_{M, 2 r}\right\} \otimes_{C} C_{x}=\left\{\left(\Delta_{\mathfrak{G}, r} \otimes M\right) \circ \Delta_{M, 2 r}\right\} \otimes_{C} C_{x}$ at each $x \in \mathfrak{X}(\mathfrak{k})$,

hence $\left(\mathfrak{i}_{r, 2 r}^{\circ} \otimes \Delta_{M, r}\right) \circ \Delta_{M, 2 r}=\left(\Delta_{\mathfrak{G}, r} \otimes M\right) \circ \Delta_{M, 2 r}$, as desired.

Assume finally that $\mathfrak{G}$ is transitive on $\mathfrak{X}$. If the hypothesis is verified at $z \in \mathfrak{X}(\mathfrak{k})$ and if $g z=x, g \in \mathfrak{G}(\mathfrak{k})$, then the equality (3) will follow from

$$
\left(\mathfrak{V}_{r}(e) \times \mathfrak{V}_{r}(e) \times g\right)^{*} \mathfrak{p}_{2, r}^{*} \phi_{2 r}=\left(\mathfrak{V}_{r}(e) \times \mathfrak{V}_{r}(e) \times g\right)^{*}\left\{\left(\mathfrak{p}_{3, r}^{*} \phi_{2 r}\right) \circ\left(\mathfrak{p}_{1, r}^{*} \phi_{2 r}\right)\right\}
$$

as the translation is invertible, hence the assertion from the hypothesis at $z$.

3.8. In the set-up of (3.4) we say $(\mathcal{M}, \phi)$ is a $\mathfrak{G}$-equivariant $\mathcal{D}_{\mathfrak{X}}$-module iff $\mathcal{M} \in \mathcal{D}_{\mathfrak{X}} \mathbf{q c}$ and $\phi \in \mathcal{D}_{\mathfrak{G} \times \mathfrak{X}} \operatorname{Mod}\left(\mathfrak{a}^{0} \mathcal{M}, \mathfrak{p}_{\mathfrak{X}}^{0} \mathcal{M}\right)^{\times}$. We will denote the category

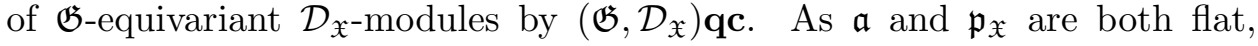
$\mathrm{L} \mathfrak{a}^{0}=\mathfrak{a}^{0}$ and $\mathrm{L} \mathfrak{p}_{\mathfrak{X}}^{0}=\mathfrak{p}_{\mathfrak{X}}^{0}$. We have as in characteristic 0 (cf. [Sa, $\left.\S 1\right]$ ).

Proposition. Let $\mathfrak{f} \in \mathbf{S c h}_{\mathfrak{k}}^{\mathfrak{G}}(\mathfrak{X}, \mathfrak{Y})$.

(i) $\quad \mathrm{Lf}^{0}: \mathrm{D}^{b}\left(\mathcal{D}_{\mathfrak{Y}} \mathbf{q} \mathbf{c}\right) \rightarrow \mathrm{D}^{b}\left(\mathcal{D}_{\mathfrak{X}} \mathbf{q} \mathbf{c}\right)$ sends $\mathrm{D}^{b}\left(\left(\mathfrak{G}, \mathcal{D}_{\mathfrak{Y}}\right) \mathbf{q} \mathbf{c}\right)$ to $\mathrm{D}^{b}\left(\left(\mathfrak{G}, \mathcal{D}_{\mathfrak{X}}\right) \mathbf{q c}\right)$.

(ii) $\int_{\mathfrak{f}}: \mathrm{D}^{b}\left(\mathcal{D}_{\mathfrak{X}} \mathbf{q} \mathbf{c}\right) \rightarrow \mathrm{D}^{b}\left(\mathcal{D}_{\mathfrak{Y}} \mathbf{q} \mathbf{c}\right)$ sends $\mathrm{D}^{b}\left(\left(\mathfrak{G}, \mathcal{D}_{\mathfrak{X}}\right) \mathbf{q c}\right)$ to $\mathrm{D}^{b}\left(\left(\mathfrak{G}, \mathcal{D}_{\mathfrak{Y}}\right) \mathbf{q} \mathbf{c}\right)$.

(iii) If $\mathfrak{Z}_{1} \supseteq \mathfrak{Z}_{2}$ are two $\mathfrak{G}$-invariant closed subsets of $\mathfrak{X}, \mathrm{R} \Gamma_{\mathfrak{Z}_{1} / \mathfrak{Z}_{2}}$ : $\mathrm{D}^{b}\left(\mathcal{D}_{\mathfrak{X}} \mathbf{q} \mathbf{c}\right) \rightarrow \mathrm{D}^{b}\left(\mathcal{D}_{\mathfrak{X}} \mathbf{q} \mathbf{c}\right)$ preserves $\mathrm{D}^{b}\left(\left(\mathfrak{G}, \mathcal{D}_{\mathfrak{X}}\right) \mathbf{q} \mathbf{c}\right)$.

Proof. (ii) An application of the base change theorem (2.12) to the cartesian square

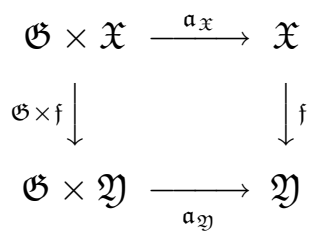

yields $\mathfrak{a}_{\mathfrak{Y}}^{0} \circ \int_{\mathfrak{f}} \simeq \int_{\mathfrak{G} \times \mathfrak{f}} \circ \mathfrak{a}_{\mathfrak{X}}^{0}$. Likewise $\mathfrak{p}_{\mathfrak{Y}}^{0} \circ \int_{\mathfrak{f}} \simeq \int_{\mathfrak{G} \times \mathfrak{f}} \circ \mathfrak{p}_{\mathfrak{Y}}^{0}$. Hence for $(\mathcal{M}, \phi) \in$ $\mathrm{D}^{b}((\mathfrak{G}, \mathfrak{X}) \mathbf{q c})$ one can define $\phi_{\int_{\mathfrak{f}} \mathcal{M}} \in \mathrm{D}^{b}\left(\mathcal{D}_{\mathfrak{G} \times \mathfrak{Y}} \mathbf{q} \mathbf{c}\right)\left(\mathfrak{a}_{\mathfrak{Y}}^{0} \int_{\mathfrak{f}} \mathcal{M}, \mathfrak{p}_{\mathfrak{Y}}^{0} \int_{\mathfrak{f}} \mathcal{M}^{\cdot}\right)^{\times}$by 
the commutative diagram

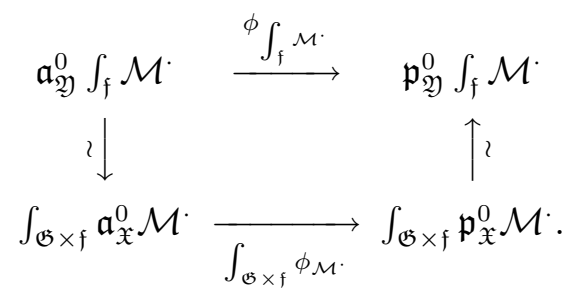

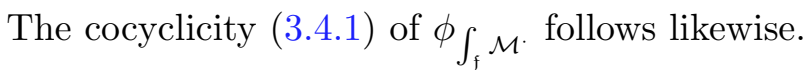

(iii) As $\mathfrak{a}_{\mathfrak{X}}=\mathfrak{p}_{\mathfrak{X}} \circ \mathfrak{a}_{\mathfrak{X}}^{\prime}$ with $\mathfrak{a}_{\mathfrak{X}}^{\prime}$ invertible, one has by $[\mathbf{K e}, 11.5]$

$$
\mathfrak{a}_{\mathfrak{X}}^{0} \circ \Gamma_{\mathfrak{Z}_{1} / \mathfrak{Z}_{2}} \simeq \Gamma_{\mathfrak{G} \times \mathfrak{Z}_{1} / \mathfrak{G} \times \mathfrak{Z}_{2}} \circ \mathfrak{a}_{\mathfrak{X}}^{0} \text { on } \mathcal{D}_{\mathfrak{X}} \mathbf{q} \mathbf{c} .
$$

Also from $[\mathrm{Ke}, 11.5]$

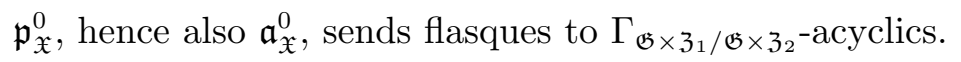

Hence on $\mathrm{D}^{b}\left(\mathcal{D}_{\mathfrak{X}} \mathbf{q} \mathbf{c}\right)$

$\mathfrak{a}_{\mathfrak{X}}^{0} \circ \mathrm{R} \Gamma_{\mathfrak{Z}_{1} / \mathfrak{Z}_{2}} \simeq \mathrm{R} \Gamma_{\mathfrak{G} \times \mathfrak{Z}_{1} / \mathfrak{G} \times \mathfrak{Z}_{2}} \circ \mathfrak{a}_{\mathfrak{X}}^{0} \quad$ and $\quad \mathfrak{p}_{\mathfrak{X}}^{0} \circ \mathrm{R} \Gamma_{\mathfrak{Z}_{1} / \mathfrak{Z}_{2}} \simeq \mathrm{R} \Gamma_{\mathfrak{G} \times \mathfrak{Z}_{1} / \mathfrak{G} \times \mathfrak{Z}_{2}} \circ \mathfrak{p}_{\mathfrak{X}}^{0}$, and the assertion follows as in (ii).

3.9. If $\mathfrak{X}$ is affine, $\mathfrak{X}$ is $\mathcal{D}$-affine by Serre's theorem, and the equivariant version of Beilinson-Bernstein's local-global principle carries over. Thus let $(\mathfrak{G}, D(\mathfrak{X}))$ Mod be the category whose objects are simultaneously $\mathfrak{G}$-modules and $D(\mathfrak{X})$-modules such that (i) the two $\operatorname{Dist}(\mathfrak{G})^{\mathrm{op}}$-actions on $M$ induced by the $\mathfrak{G}$-module structure and the $D(\mathfrak{X})$-module structure coincide, i.e., if $\Delta_{M}: M \rightarrow M \otimes \mathfrak{k}[\mathfrak{G}]$ is the $\mathfrak{G}$-module structure on $M$, then with $\phi^{\prime}$ : $\operatorname{Dist}(\mathfrak{G})^{\text {op }} \rightarrow D(\mathfrak{X})$ of $(3.6)$ and transposition $\tau: M \otimes \mathfrak{k}[\mathfrak{G}] \rightarrow \mathfrak{k}[\mathfrak{G}] \otimes M$

$$
(\mu \otimes M) \circ \tau \circ \Delta_{M}=\phi^{\prime}(\mu) \quad \forall \mu \in \operatorname{Dist}(\mathfrak{G}),
$$

and that (ii) $\forall \delta \in D(\mathfrak{X}), g \in \mathfrak{G}$ and $m \in M, g(\delta m)=(g \delta)(g m)$.

Proposition (cf. [Bø, Prop. 4.5]). If $\mathfrak{X}$ is affine, there is an equivalence of categories $\left(\mathfrak{G}, \mathcal{D}_{\mathfrak{X}}\right) \mathbf{q c} \rightarrow(\mathfrak{G}, D(\mathfrak{X})) \operatorname{Mod}$ via $\mathcal{M} \mapsto \mathcal{M}(\mathfrak{X})$ with quasi-inverse $M \mapsto\left(\mathcal{D}_{\mathfrak{X}} \otimes_{D(\mathfrak{X})} M, \phi\right)$ such that $\phi(\mathfrak{G} \times \mathfrak{X}) \in$

$$
\begin{aligned}
& D(\mathfrak{G} \times \mathfrak{X}) \operatorname{Mod}\left(\left(\mathfrak{k}[\mathfrak{G} \times \mathfrak{X}], \mathfrak{a}^{\circ}\right) \otimes_{\mathfrak{k}[\mathfrak{X}]} D(\mathfrak{X}) \otimes_{D(\mathfrak{X})} M,\right. \\
&\left.\left(\mathfrak{k}[\mathfrak{G} \times \mathfrak{X}], \mathfrak{p}^{\circ}\right) \otimes_{\mathfrak{k}[\mathfrak{X}]} D(\mathfrak{X}) \otimes_{D(\mathfrak{X})} M\right)
\end{aligned}
$$


is induced by the left $\mathfrak{k}[\mathfrak{G}]$-comodule $\operatorname{map} \tau \circ \Delta_{M}: M \rightarrow \mathfrak{k}[\mathfrak{G}] \otimes M$.

\subsection{0 .}

Remark. If $\mathfrak{X}$ is not affine, the proposition implies for each affine open $\mathfrak{U}$ of $\mathfrak{X}$ and for each infinitesimal subgroup $\mathfrak{G}_{r}$ of $\mathfrak{G}, r \in \mathbb{N}$, that there is an equivalence

$$
\left(\mathfrak{G}_{r}, \mathcal{D}_{\mathfrak{U}}\right) \mathbf{q c} \rightarrow\left(\mathfrak{G}_{r}, D(\mathfrak{U})\right) \text { Mod }
$$

3.11. If $\mathfrak{X}$ is a $\mathfrak{G}$-variety, $\mathcal{O}_{\mathfrak{X}}$ is naturally equipped with a structure of $\left(\mathfrak{G}, \mathcal{D}_{\mathfrak{X}}\right) \mathbf{q c}$, hence also $\mathrm{R}^{i} \Gamma_{\mathfrak{Z}_{1} / \mathfrak{Z}_{2}}\left(\mathcal{O}_{\mathfrak{X}}\right)$ for each $i \in \mathbb{N}$ and two closed subsets $\mathfrak{Z}_{1} \supseteq \mathfrak{Z}_{2}$ of $\mathfrak{X}$.

Using either the standard filtration or the $p$-filtration, one can equip $\mathcal{D}_{\mathfrak{X}}$ with a structure of $(\mathfrak{G}, \mathfrak{X}) \mathbf{q c}$ as follows. Let $\Delta_{\mathfrak{X}}: \mathfrak{X} \rightarrow \mathfrak{X} \times \mathfrak{X}$ be the diagonal immersion, $\mathfrak{I}_{\mathfrak{X}}$ the ideal sheaf of the closed subvariety $\operatorname{im}\left(\Delta_{\mathfrak{X}}\right)$ in $\mathfrak{X} \times \mathfrak{X}, \mathfrak{X}_{\Delta, n}=\left(\left|\operatorname{im} \Delta_{\mathfrak{X}}\right|, \mathcal{O}_{\mathfrak{X} \times \mathfrak{X}} / \mathfrak{I}_{\mathfrak{X}}^{n+1}\right)$ the $\mathrm{n}$-th infinitesimal neighbourhood of $\operatorname{im}\left(\Delta_{\mathfrak{X}}\right)$, and $\mathfrak{p}_{i, n}=\mathfrak{p}_{i}$ ○ inc : $\mathfrak{X}_{\Delta, n} \rightarrow \mathfrak{X}, i=1,2$, with $\mathfrak{p}_{i}: \mathfrak{X} \times \mathfrak{X} \rightarrow \mathfrak{X}$ the i-th projection. By [EGAIV, 16.7.1.1]

$$
\mathcal{P}_{\mathfrak{X}}^{n}=\left(\mathfrak{p}_{1, n}\right)_{*} \mathfrak{p}_{2, n}^{*} \mathcal{O}_{\mathfrak{X}} .
$$

As $\operatorname{im}\left(\Delta_{\mathfrak{X}}\right)$ is $\mathfrak{G}$-invariant, so is $\mathfrak{X}_{\Delta, n}$, hence $\mathfrak{p}_{i, n}$ are both $\mathfrak{G}$-equivariant. Then $\mathcal{P}_{\mathfrak{X}}^{n}$ inherits a structure of $(\mathfrak{G}, \mathfrak{X}) \mathbf{q c}$ from $\mathcal{O}_{\mathfrak{X}}$. As $\mathcal{P}_{\mathfrak{X}}^{n}$ is locally free of finite rank in $\mathfrak{X M o d}$, one has

$$
\mathfrak{a}^{*} \mathcal{D} i f f_{\mathfrak{X}}^{n} \simeq \mathfrak{a}^{*} \operatorname{Mod}_{\mathfrak{X}}\left(\mathcal{P}_{\mathfrak{X}}^{n}, \mathcal{O}_{\mathfrak{X}}\right) \simeq \operatorname{Mod}_{\mathfrak{G} \times \mathfrak{X}}\left(\mathfrak{a}^{*} \mathcal{P}_{\mathfrak{X}}^{n}, \mathfrak{a}^{*} \mathcal{O}_{\mathfrak{X}}\right)
$$

and likewise with $\mathfrak{p}_{\mathfrak{X}}^{*}$. Hence from the structure morphism $\phi_{\mathcal{P}_{\mathfrak{X}}^{n}}$ one can define a structure of $(\mathfrak{G}, \mathfrak{X}) \mathbf{q c}$ on $\mathcal{D} i f f_{\mathfrak{X}}^{n}$ by the composite

$$
\begin{aligned}
\operatorname{Mod}_{\mathfrak{G} \times \mathfrak{X}}\left(\mathfrak{p}_{\mathfrak{X}}^{*} \mathcal{P}_{\mathfrak{X}}^{n}, \phi_{\mathcal{O}_{\mathfrak{X}}}\right) \circ \mathcal{M o d}_{\mathfrak{G} \times \mathfrak{X}}\left(\phi_{\mathcal{P}_{\mathfrak{X}}^{n}}^{-1}, \mathfrak{a}^{*} \mathcal{O}_{\mathfrak{X}}\right): & \\
\mathcal{M o d}_{\mathfrak{G} \times \mathfrak{X}}\left(\mathfrak{a}^{*} \mathcal{P}_{\mathfrak{X}}^{n}, \mathfrak{a}^{*} \mathcal{O}_{\mathfrak{X}}\right) & \rightarrow \operatorname{Mod}_{\mathfrak{G} \times \mathfrak{X}}\left(\mathfrak{p}_{\mathfrak{X}}^{*} \mathcal{P}_{\mathfrak{X}}^{n}, \mathfrak{p}_{\mathfrak{X}}^{*} \mathcal{O}_{\mathfrak{X}}\right) .
\end{aligned}
$$

Then $\underline{\lim }_{\underline{n}} \phi_{\mathcal{D} i f f_{\mathfrak{x}}^{n}}$ makes $\mathcal{D}_{\mathfrak{X}}$ into a $\mathfrak{G}$-equivariant $\mathcal{O}_{\mathfrak{X}}$-module. Equivalently, one could define a structure of $(\mathfrak{G}, \mathfrak{X}) \mathbf{q c}$ on $\mathcal{D}_{\mathfrak{X}, r} \simeq \operatorname{Mod}_{\mathfrak{X}}\left(\left(\mathfrak{F}_{\mathfrak{X}}^{r}\right)^{*}\left(\mathfrak{F}_{\mathfrak{X}}^{r}\right)_{*} \mathcal{O}_{\mathfrak{X}}, \mathcal{O}_{\mathfrak{X}}\right)$ $[\mathbf{H} 87,1.2 .6]$ by the composite

$$
\operatorname{Mod}_{\mathfrak{G} \times \mathfrak{X}}\left(\mathfrak{p}_{\mathfrak{X}}^{*}\left(\mathfrak{F}_{\mathfrak{X}}^{r}\right)^{*}\left(\mathfrak{F}_{\mathfrak{X}}^{r}\right)_{*} \mathcal{O}_{\mathfrak{X}}, \phi_{\mathcal{O}_{\mathfrak{X}}}\right) \circ \mathcal{M o d}_{\mathfrak{G} \times \mathfrak{X}}\left(\phi_{\left(\mathfrak{F}_{\mathfrak{X}}^{r}\right)^{*}\left(\mathfrak{F}_{\mathfrak{X}}^{r}\right)_{*} \mathcal{O}_{\mathfrak{X}}}^{-1} \mathfrak{a}^{*} \mathcal{O}_{\mathfrak{X}}\right),
$$

and take $\underline{\lim }_{\phi_{\mathcal{D}_{\mathfrak{x}, r}}}$. Unfortunately,

$$
\phi_{\mathcal{D}_{\mathfrak{X}}} \text { is not } \mathcal{D}_{\mathfrak{G} \times \mathfrak{X}} \text {-equivariant, hence }\left(\mathcal{D}_{\mathfrak{X}}, \phi_{\mathcal{D}_{\mathfrak{X}}}\right) \notin\left(\mathfrak{G}, \mathcal{D}_{\mathfrak{X}}\right) \mathbf{q c} \text {. }
$$


Explicitly, let us consider $\phi_{\mathcal{D}_{\mathfrak{x}, r}}$ on $\mathfrak{G}_{r} \times \mathfrak{U}, r \in \mathbb{N}, \mathfrak{U}$ affine open of $\mathfrak{X}$. Let $\mathfrak{i}: G_{r} \hookrightarrow \mathfrak{G}, \mathfrak{j}: \mathfrak{U} \hookrightarrow \mathfrak{X}, \mathfrak{a}_{r}=\mathfrak{a} \circ(\mathfrak{i} \times \mathfrak{j}), \mathfrak{p}_{r}=\mathfrak{p}_{\mathfrak{X}} \circ(\mathfrak{i} \times \mathfrak{j}), \Phi=\left((\mathfrak{i} \times \mathfrak{j})^{*} \phi_{\mathcal{O}_{\mathfrak{X}}}\right)\left(\mathfrak{G}_{r} \times\right.$ $\mathfrak{U}), \Phi^{\prime}=\left((\mathfrak{i} \times \mathfrak{j})^{*} \phi_{\left(\mathfrak{F}_{\mathfrak{x}}^{r}\right)^{*}\left(\mathfrak{F}_{\mathfrak{x}}^{r}\right)_{*} \mathcal{O}_{\mathfrak{x}}}\right)\left(\mathfrak{G}_{r} \times \mathfrak{U}\right),\left(\Phi^{\prime}, \Phi\right)=\left((\mathfrak{i} \times \mathfrak{j})^{*} \phi_{\mathcal{D}_{\mathfrak{x}, r}}\right)\left(\mathfrak{G}_{r} \times \mathfrak{U}\right)$, $A=\mathfrak{k}\left[\mathfrak{G}_{r}\right]$, and $C=\mathfrak{k}[\mathfrak{U}]$. Then $\Phi:\left(A \otimes C, \mathfrak{a}_{r}^{\circ}\right) \otimes_{C} C \rightarrow A \otimes C$ is given by $a \otimes b \otimes c \longmapsto(a \otimes b) \mathfrak{a}_{r}^{\circ}(c)$, hence $\Phi^{\prime}:\left(A \otimes C^{(r)}, \mathfrak{a}_{r}^{\circ}\right) \otimes_{C^{(r)}} C \rightarrow(A \otimes$ $\left.C, \mathfrak{p}_{r}^{\mathrm{o}}\right) \otimes_{C^{(r)}} C$ by $a \otimes b \otimes c \longmapsto \sum a a_{i} \otimes b \otimes c_{i}$ if $\mathfrak{a}_{r}^{\mathrm{o}}(c)=\sum a_{i} \otimes c_{i}$. Then $\left(\Phi^{\prime}\right)^{-1}: a \otimes b \otimes c \longmapsto \sum a \sigma\left(a_{i}\right) \otimes b \otimes c_{i}$ with $\sigma$ the antipode of $A$, hence $\left(\Phi^{\prime}, \Phi\right):\left(A \otimes C, \mathfrak{a}^{\circ}\right) \otimes_{C} D_{r}(C) \rightarrow\left(A \otimes C, \mathfrak{p}^{\circ}\right) \otimes_{C} D_{r}(C)$ is given by

$$
f \longmapsto(\sigma \bar{\otimes} f) \circ \mathfrak{a}_{r}^{\circ}=(\mathfrak{m u l t} \otimes C) \circ(\sigma \otimes f) \circ \mathfrak{a}_{r}^{o}
$$

upon identification of $\left(A \otimes C, \mathfrak{a}^{\circ}\right) \otimes_{C} D_{r}(C)$ (resp. $\left.\left(A \otimes C, \mathfrak{p}^{\circ}\right) \otimes_{C} D_{r}(C)\right)$ with $D_{r}(C, A \otimes C)$. In particular, the $\mathfrak{G}_{r}^{\text {op }}$-action on $D_{r}(C)$ is given by

$$
(x \cdot \theta)(c)=x\left(\theta\left(x^{-1} c\right)\right), \quad x \in \mathfrak{G}_{r}, c \in C,
$$

where the RHS is written with respect to the $\mathfrak{G}_{r}^{\text {op }}$-action on $C$ given by $x c=(x \otimes C) \circ \mathfrak{a}_{r}^{\circ}(c)$ in $R \otimes C$ if $x \in \mathfrak{G}_{r}(R), R \in \mathbf{A l g}_{\mathfrak{k}}$. If $\delta_{1} \otimes \delta_{2} \in$ $D_{r}\left(\mathfrak{G}_{r}\right) \otimes D_{r}(C)=D_{r}(A \otimes C)$, then

$$
\left\{\sigma \bar{\otimes}\left(\left(\delta_{1} \otimes \delta_{2}\right) \circ f\right)\right\} \circ \mathfrak{a}_{r}^{\circ}(c)=\sum \sigma\left(a_{i}\right)\left(\delta_{1} \otimes \delta_{2}\right) f\left(c_{i}\right)
$$

while

$$
\left(\delta_{1} \otimes \delta_{2}\right) \circ(\sigma \bar{\otimes} f) \circ \mathfrak{a}_{r}^{\circ}(c)=\sum\left(\delta_{1} \otimes \delta_{2}\right)\left(\sigma\left(a_{i}\right) f\left(c_{i}\right)\right) .
$$

Hence $\phi_{\mathcal{D}_{\mathfrak{X}, r}}$ is only $\mathcal{O}_{\mathfrak{G}} \otimes \mathcal{D}_{\mathfrak{X}, r}$-linear, i.e., $\mathcal{D}_{\mathfrak{X}}$ is a quasi-Gr-equivariant $\mathcal{D}_{\mathfrak{X}^{-}}$ module [Ka, 4.7]. We will denote the category of quasi-G $\mathfrak{G}$-equivariant $\mathcal{D}_{\mathfrak{X}^{-}}$ modules by $\left(\mathfrak{G}, \mathcal{D}_{\mathfrak{X}}\right) \mathbf{q} q c$.

Nevertheless, in [Bø, Prop. 4.5] it is proved that if $\mathfrak{X}=G / B$ is a flag variety with $G$ semisimple and if $M$ is a $D(\mathfrak{X})$-module such that the induced $\operatorname{Dist}(G)^{\mathrm{op}}$-action on $M$ lifts to make a $G^{\mathrm{op}}$-module, then $\mathcal{D}_{\mathfrak{X}} \otimes_{D(\mathfrak{X})} M$ admits a structure of $\left(G, \mathcal{D}_{\mathfrak{X}}\right) \mathbf{q c}$.

3.12. Resume the notation of (2.2). We define two equivariant versions of $\mathfrak{X}^{\infty}$-modules, categories $\left(\mathfrak{G}, \mathfrak{X}^{\infty}\right) \mathbf{q c}$ and $\left(\mathfrak{G}, \mathfrak{X}^{\infty}\right) \mathbf{q q} \mathbf{c}$ of $\mathfrak{G}$-equivariant $\mathfrak{X}^{\infty}$ modules and quasi- $\mathfrak{G}$-equivariant $\mathfrak{X}^{\infty}$-modules, respectively.

Definition. A $\mathfrak{G}$-equivariant $\mathfrak{X}^{\infty}$-module is a projective system $\left(\mathcal{M}^{(r)}, \phi^{(r)}\right)_{r \in \mathbb{N}}$ of $\mathfrak{X}^{\infty}$-module $\left(\mathcal{M}^{(r)}\right)_{r}$ such that

$$
\left(\mathcal{M}^{(r)}, \phi^{(r)}\right) \in\left(\mathfrak{G}^{(r)}, \mathfrak{X}^{(r)}\right) \mathbf{q c}
$$

and that for each $r \in \mathbb{N}$ the structure morphism $\gamma_{r+1, r} \in \operatorname{Mod}_{\mathcal{O}_{X}^{(r+1)}}\left(\mathcal{M}^{(r+1)}\right.$, $\left.\mathcal{M}^{(r)}\right)$ of the projective system $\left(\mathcal{M}^{(r)}\right)_{r}$ induces an isomorphism

$$
\mathcal{O}_{\mathfrak{X}}^{(r)} \otimes_{\mathcal{O}_{\mathfrak{X}}^{(r+1)}} \mathcal{M}^{(r+1)} \rightarrow \mathcal{M}^{(r)} \quad \text { in }\left(\mathfrak{G}^{(r)}, \mathfrak{X}^{(r)}\right) \mathbf{q} \mathbf{c},
$$


i.e., identifying $\mathfrak{X}^{(r)}$ with $\left(\mathfrak{X}, \mathcal{O}_{\mathfrak{X}}^{(r)}\right)$ the composite

$$
\begin{aligned}
& \left(\mathfrak{a}^{(r)}\right)^{*}\left(\mathcal{O}_{\mathfrak{X}}^{(r)} \otimes_{\mathcal{O}_{\mathfrak{X}}^{(r+1)}} \mathcal{M}^{(r+1)}\right) \stackrel{\left(\mathfrak{a}^{(r)}\right)^{*}\left(\mathcal{O}_{\mathfrak{X}}^{(r)} \otimes_{\left.\mathcal{O}_{X}^{(r+1)} \gamma_{r+1, r}\right)}\right.}{\longrightarrow} \\
& \left(\mathfrak{a}^{(r)}\right)^{*}\left(\mathcal{M}^{(r)}\right) \stackrel{\phi^{(r)}}{\longrightarrow}\left(\mathfrak{p}_{\mathfrak{X}}^{(r)}\right)^{*}\left(\mathcal{M}^{(r)}\right)
\end{aligned}
$$

is equal in $\operatorname{Mod}_{(\mathfrak{G} \times \mathfrak{X})^{(r)}}$ to the composite

$$
\begin{aligned}
& \left(\mathfrak{a}^{(r)}\right)^{*}\left(\mathcal{O}_{\mathfrak{X}}^{(r)} \otimes_{\mathcal{O}_{\mathfrak{X}}^{(r+1)}} \mathcal{M}^{(r+1)}\right) \\
& \stackrel{\sim}{\longrightarrow}\left(\mathfrak{a}^{(r)}\right)^{*} \mathfrak{F}_{\mathfrak{X}^{(r)}}^{*}\left(\mathcal{M}^{(r+1)}\right) \stackrel{\sim}{\longrightarrow} \mathfrak{F}_{(\mathfrak{G} \times \mathfrak{X})^{(r)}}^{*}\left(\mathfrak{a}^{(r+1)}\right)^{*}\left(\mathcal{M}^{(r+1)}\right) \\
& \stackrel{\mathfrak{F}_{(\mathfrak{G} \times \mathfrak{X})^{(r)}}^{*}\left(\phi^{(r+1)}\right)}{\longrightarrow} \mathfrak{F}_{(\mathfrak{G} \times \mathfrak{X})^{(r)}}^{*}\left(\mathfrak{p}_{\mathfrak{X}}^{(r+1)}\right)^{*}\left(\mathcal{M}^{(r+1)}\right) \\
& \stackrel{\sim}{\longrightarrow}\left(\mathfrak{p}_{\mathfrak{X}}^{(r)}\right)^{*}\left(\mathcal{O}_{\mathfrak{X}}^{(r)} \otimes_{\mathcal{O}_{\mathfrak{X}}^{(r+1)}} \mathcal{M}^{(r+1)}\right) \\
& \stackrel{\left(\mathfrak{p}_{\mathfrak{G} \times \mathfrak{X}}^{(r)}\right)^{*}\left(\mathcal{O}_{\mathfrak{X}}^{(r)} \otimes_{\left.\mathcal{O}_{\mathfrak{X}}^{(r+1)} \gamma_{r+1, r}\right)}^{\longrightarrow}\right.}{\longrightarrow}\left(\mathfrak{p}_{\mathfrak{X}}^{(r)}\right)^{*}\left(\mathcal{M}^{(r)}\right) .
\end{aligned}
$$

A quasi-G-equivariant $\mathfrak{X}^{\infty}$-module is a projective system $\left(\mathcal{N}^{(r)}, \phi_{r}\right)_{r \in \mathbb{N}}$ of $\mathfrak{X}^{\infty}$-module $\left(\mathcal{N}^{(r)}\right)_{r}$ such that

$$
\left(\mathcal{N}^{(r)}, \phi_{r}\right) \in\left(\mathfrak{G}, \mathfrak{X}^{(r)}\right) \mathbf{q c}
$$

and that for each $r$ the structure morphism $\eta_{r+1, r}: \mathcal{N}^{(r+1)} \rightarrow \mathcal{N}^{(r)}$ of the projective system $\left(\mathcal{N}^{(r)}\right)_{r}$ induces an isomorphism

$$
\mathcal{O}_{\mathfrak{X}}^{(r)} \otimes_{\mathcal{O}_{\mathfrak{X}}^{(r+1)}} \mathcal{N}^{(r+1)} \rightarrow \mathcal{N}^{(r)} \quad \text { in }\left(\mathfrak{G}, \mathfrak{X}^{(r)}\right) \mathbf{q} \mathbf{c},
$$

i.e., if $\mathfrak{a}_{r}=\mathfrak{a}^{(r)} \circ\left(\mathfrak{F}_{\mathfrak{G}}^{r} \times \mathfrak{X}^{(r)}\right)$ and $\mathfrak{p}_{r}=\mathfrak{p}_{\mathfrak{X}}^{(r)} \circ\left(\mathfrak{F}_{\mathfrak{G}}^{r} \times \mathfrak{X}^{(r)}\right): \mathfrak{G} \times \mathfrak{X}^{(r)} \rightarrow \mathfrak{X}^{(r)}$, the composite

$$
\begin{aligned}
& \left(\mathfrak{a}_{r}\right)^{*}\left(\mathcal{O}_{\mathfrak{X}}^{(r)} \otimes_{\mathcal{O}_{\mathfrak{X}}^{(r+1)}} \mathcal{N}^{(r+1)}\right) \stackrel{\left(\mathfrak{a}_{r}\right)^{*}\left(\mathcal{O}_{\mathfrak{X}}^{(r)} \otimes_{\mathcal{O}_{X}^{(r+1)}} \eta_{r+1, r}\right)}{\longrightarrow} \\
& \left(\mathfrak{a}_{r}\right)^{*}\left(\mathcal{N}^{(r)}\right) \stackrel{\phi_{r}}{\longrightarrow}\left(\mathfrak{p}_{r}\right)^{*}\left(\mathcal{N}^{(r)}\right)
\end{aligned}
$$

is equal in $\operatorname{Mod}_{\mathfrak{G} \times \mathfrak{X}^{(r)}}$ to the composite

$$
\begin{aligned}
\left(\mathfrak{a}_{r}\right)^{*}\left(\mathcal{O}_{\mathfrak{X}}^{(r)} \otimes_{\mathcal{O}_{\mathfrak{X}}^{(r+1)}} \mathcal{N}^{(r+1)}\right) & \stackrel{\sim}{\longrightarrow}\left(\mathfrak{G} \times \mathfrak{F}_{\mathfrak{X}(r)}\right)^{*}\left(\mathfrak{a}_{r+1}\right)^{*}\left(\mathcal{N}^{(r+1)}\right) \\
& \stackrel{\left(\mathfrak{G} \times \mathfrak{F}_{\mathfrak{X}}(r)\right)^{*}\left(\phi_{r+1}\right)}{\longrightarrow}\left(\mathfrak{G} \times \mathfrak{F}_{\mathfrak{X}(r)}\right)^{*}\left(\mathfrak{p}_{r+1}\right)^{*}\left(\mathcal{N}^{(r+1)}\right) \\
& \stackrel{\sim}{\longrightarrow}\left(\mathfrak{p}_{r}\right)^{*}\left(\mathcal{O}_{\mathfrak{X}}^{(r)} \otimes_{\mathcal{O}_{X}^{(r+1)}} \mathcal{N}^{(r+1)}\right) \\
& \stackrel{\left(\mathfrak{p}_{r}\right)^{*}\left(\mathcal{O}_{\mathfrak{X}}^{(r)} \otimes_{\mathcal{O}_{\mathfrak{X}}^{(r+1)}} \eta_{r+1, r}\right.}{\longrightarrow}\left(\mathfrak{p}_{r}\right)^{*}\left(\mathcal{N}^{(r)}\right)
\end{aligned}
$$


3.13. If $(\mathcal{M}, \phi) \in\left(\mathfrak{G}, \mathcal{D}_{\mathfrak{X}}\right) \mathbf{q c}$, then by the Cartier-Chase-Smith equivalence $[\mathbf{H 8 7}, 2.2 .1]$ with $\mathcal{M}^{(r)}=\operatorname{Mod}_{\mathcal{O}_{\mathfrak{X}}^{(r)}}\left(\mathcal{O}_{\mathfrak{X}}, \mathcal{O}_{\mathfrak{X}}^{(r)}\right) \otimes_{\mathcal{D}_{\mathfrak{X}, r}} \mathcal{M}$

$$
\begin{aligned}
\left(\mathfrak{a}^{(r)}\right)^{*}\left(\mathcal{M}^{(r)}\right) & \simeq \operatorname{Mod}_{\mathcal{O}_{\mathfrak{G} \times \mathfrak{X}}^{(r)}}\left(\mathcal{O}_{\mathfrak{G} \times \mathfrak{X}}, \mathcal{O}_{\mathfrak{G} \times \mathfrak{X}}^{(r)}\right) \otimes_{\mathcal{D}_{\mathfrak{G} \times \mathfrak{X}, r}}\left(\mathfrak{F}_{\mathfrak{G} \times \mathfrak{X}}^{r}\right)^{*}\left(\mathfrak{a}^{(r)}\right)^{*}\left(\mathcal{M}^{(r)}\right) \\
& \simeq \mathcal{M o d}_{\mathcal{O}_{\mathfrak{G} \times \mathfrak{X}}^{(r)}}\left(\mathcal{O}_{\mathfrak{G} \times \mathfrak{X}}, \mathcal{O}_{\mathfrak{G} \times \mathfrak{X}}^{(r)}\right) \otimes_{\mathcal{D}_{\mathfrak{B} \times \mathfrak{X}, r}} \mathfrak{a}^{*}\left(\mathfrak{F}_{\mathfrak{X}}^{r}\right)^{*}\left(\mathcal{M}^{(r)}\right) \\
& \simeq \mathcal{M o d}_{\mathcal{O}_{\mathfrak{G} \times \mathfrak{X}}^{(r)}}\left(\mathcal{O}_{\mathfrak{G} \times \mathfrak{X}}, \mathcal{O}_{\mathfrak{G} \times \mathfrak{X}}^{(r)}\right) \otimes_{\mathcal{D}_{\mathfrak{G} \times \mathfrak{X}, r}} \mathfrak{a}^{*} \mathcal{M}
\end{aligned}
$$

and likewise

$$
\left(\mathfrak{p}_{\mathfrak{X}}^{(r)}\right)^{*}\left(\mathcal{M}^{(r)}\right) \simeq \operatorname{Mod}_{\mathcal{O}_{\mathfrak{G} \times \mathfrak{X}}^{(r)}}\left(\mathcal{O}_{\mathfrak{G} \times \mathfrak{X}}, \mathcal{O}_{\mathfrak{G} \times \mathfrak{X}}^{(r)}\right) \otimes_{\mathcal{D}_{\mathfrak{G} \times \mathfrak{X}, r}} \mathfrak{p}_{\mathfrak{X}}^{*} \mathcal{M}
$$

Under these identifications define

$$
\phi^{(r)} \in \operatorname{Mod}_{\mathcal{O}_{\mathfrak{G} \times \mathfrak{X}}^{(r)}}\left(\left(\mathfrak{a}^{(r)}\right)^{*}\left(\mathcal{M}^{(r)}\right),\left(\mathfrak{p}_{\mathfrak{X}}^{(r)}\right)^{*}\left(\mathcal{M}^{(r)}\right)\right)^{\times}
$$

to be $\operatorname{Mod}_{\mathcal{O}_{\mathfrak{G} \times \mathfrak{X}}^{(r)}}\left(\mathcal{O}_{\mathfrak{G} \times \mathfrak{X}}, \mathcal{O}_{\mathfrak{G} \times \mathfrak{X}}^{(r)}\right) \otimes_{\mathcal{D}_{\mathfrak{G} \times \mathfrak{X}, r}} \phi$. Then $\left(\mathcal{M}^{(r)}, \phi^{(r)}\right)_{r}$ forms a $\mathfrak{G}$ equivariant $\mathfrak{X}^{\infty}$-module. To check the condition (3.12.2), recall that

$$
\begin{aligned}
\mathcal{O}_{\mathfrak{G} \times \mathfrak{X}}^{(r)} \otimes_{\mathcal{O}_{\mathfrak{G} \times \mathfrak{X}}^{(r+1)}} & \operatorname{Mod}_{\mathcal{O}_{\mathfrak{G} \times \mathfrak{X}}^{(r+1)}}\left(\mathcal{O}_{\mathfrak{G} \times \mathfrak{X}}, \mathcal{O}_{\mathfrak{G} \times \mathfrak{X}}^{(r+1)}\right) \otimes_{\mathcal{D}_{\mathfrak{G} \times \mathfrak{X}, r+1}} \mathcal{L} \\
& \simeq \mathcal{M o d}_{\mathcal{O}_{\mathfrak{G} \times \mathfrak{X}}^{(r)}}\left(\mathcal{O}_{\mathfrak{G} \times \mathfrak{X}}, \mathcal{O}_{\mathfrak{G} \times \mathfrak{X}}^{(r)}\right) \otimes_{\mathcal{D}_{\mathfrak{G} \times \mathfrak{X}, r}} \mathcal{L} \quad \forall \mathcal{L} \in \mathcal{D}_{\mathfrak{G} \times \mathfrak{X}} \mathbf{q} \mathbf{c}
\end{aligned}
$$

as applying $\mathcal{O}_{\mathfrak{G} \times \mathfrak{X}} \otimes_{\mathcal{O}_{\mathfrak{G} \times \mathfrak{x}}^{(r)}}$ ? on both sides yields an isomorphism and as $\mathcal{O}_{\mathfrak{G} \times \mathfrak{X}}$ is faithfully flat over $\mathcal{O}_{\mathfrak{G} \times \mathfrak{X}}^{(r)}$. One then obtains a commutative diagram

$$
\begin{aligned}
& \left(\mathfrak{a}^{(r)}\right)^{*}\left(\mathcal{O}_{\mathfrak{X}}^{(r)} \otimes_{\mathcal{O}_{\mathfrak{X}}^{(r+1)}} \mathcal{M}^{(r+1)}\right) \longrightarrow \quad\left(\mathfrak{a}^{(r)}\right)^{*}\left(\mathcal{M}^{(r)}\right) \\
& \mathfrak{a}_{r}^{*}\left(\mathcal{N}^{(r)}\right) \\
& \simeq \operatorname{Mod}_{\mathcal{O}_{\mathfrak{G}} \otimes \mathcal{O}_{\mathfrak{X}}^{(r)}}\left(\mathcal{O}_{\mathfrak{G}} \otimes \mathcal{O}_{\mathfrak{X}}, \mathcal{O}_{\mathfrak{G}} \otimes \mathcal{O}_{\mathfrak{X}}^{(r)}\right) \otimes_{\mathcal{O}_{\mathfrak{G}} \otimes \mathcal{D} \mathfrak{X}, r}\left(\mathfrak{G} \times \mathfrak{F}_{\mathfrak{X}}^{r}\right)^{*} \mathfrak{a}_{r}^{*}\left(\mathcal{N}^{(r)}\right) \\
& \simeq \operatorname{Mod}_{\mathcal{O}_{\mathfrak{G}} \otimes \mathcal{O}_{\mathfrak{X}}^{(r)}}\left(\mathcal{O}_{\mathfrak{G}} \otimes \mathcal{O}_{\mathfrak{X}}, \mathcal{O}_{\mathfrak{G}} \otimes \mathcal{O}_{\mathfrak{X}}^{(r)}\right) \otimes_{\mathcal{O}_{\mathfrak{G}} \otimes \mathcal{D}_{\mathfrak{X}, r}} \mathfrak{a}^{*} \mathcal{N} \\
& \simeq\left\{\mathcal{O}_{\mathfrak{G}} \otimes \mathcal{M o d}_{\mathcal{O}_{\mathfrak{X}}^{(r)}}\left(\mathcal{O}_{\mathfrak{X}}, \mathcal{O}_{\mathfrak{X}}^{(r)}\right)\right\} \otimes_{\mathcal{O}_{\mathfrak{G}} \otimes \mathcal{D}_{\mathfrak{X}, r}} \mathfrak{a}^{*} \mathcal{N},
\end{aligned}
$$


and likewise

$$
\mathfrak{p}_{r}^{*}\left(\mathcal{N}^{(r)}\right) \simeq\left\{\mathcal{O}_{\mathfrak{G}} \otimes \mathcal{M o d}_{\mathcal{O}_{\mathfrak{X}}^{(r)}}\left(\mathcal{O}_{\mathfrak{X}}, \mathcal{O}_{\mathfrak{X}}^{(r)}\right)\right\} \otimes_{\mathcal{O}_{\mathfrak{G}} \otimes \mathcal{D}_{\mathfrak{X}, r}} \mathfrak{p}_{\mathfrak{X}}^{*} \mathcal{N}
$$

Hence one can define $\psi_{r} \in \operatorname{Mod}_{\mathcal{O}_{\mathfrak{s}} \otimes \mathcal{O}_{\mathfrak{x}}^{(r)}}\left(\mathfrak{a}_{r}^{*}\left(\mathcal{N}^{(r)}\right), \mathfrak{p}_{r}^{*}\left(\mathcal{N}^{(r)}\right)\right)^{\times}$from $\psi$. Then $\left(\mathcal{N}^{(r)}, \psi_{r}\right)$ forms a quasi-Ǵ-equivariant $\mathfrak{X}^{(r)}$-module. Hence $[\mathbf{H} 87,2.2 .4]$ is now refined to:

Theorem. Let $\mathcal{C}\left(\mathfrak{X}^{\infty}\right)$ be the category of $\mathfrak{X}^{\infty}$-modules. The equivalence of categories $\mathcal{D}_{\mathfrak{X}} \mathbf{q} \mathbf{c} \rightarrow \mathcal{C}\left(\mathfrak{X}^{\infty}\right)$ via $\mathcal{M} \mapsto\left(\mathcal{M}^{(r)}\right)_{r}$ induces upon restriction two equivalences $\left(\mathfrak{G}, \mathcal{D}_{\mathfrak{X}}\right) \mathbf{q} \mathbf{c} \rightarrow\left(\mathfrak{G}, \mathfrak{X}^{\infty}\right) \mathbf{q c}$ and $\left(\mathfrak{G}, \mathcal{D}_{\mathfrak{X}}\right) \mathbf{q q} \mathbf{c} \rightarrow\left(\mathfrak{G}, \mathfrak{X}^{\infty}\right) \mathbf{q q} \mathbf{c}$.

3.14. Assume $\mathfrak{G}$ is connected and reduced. Let $\mathfrak{H}$ be a closed subgroup scheme of $\mathfrak{G}$ so that the quotient $\mathfrak{G} / \mathfrak{H}$ is a $\mathfrak{G}$-variety via the multiplication from the left. For $M \in \mathfrak{H} \operatorname{Mod}$ let $\mathcal{L}_{\mathfrak{G} / \mathfrak{H}}(M)$ be the $\mathcal{O}_{\mathfrak{G} / \mathfrak{H}}$-module associated to $M$ [J, I.5]. It is well-known that

(1) $\mathcal{L}_{\mathfrak{G} / \mathfrak{H}}$ defines a functor $\mathfrak{H}$ Mod $\rightarrow(\mathfrak{G}, \mathfrak{G} / \mathfrak{H}) \mathbf{q c}$, that is an equivalence

$$
\text { with quasi-inverse } \mathcal{M} \mapsto \mathcal{M}(e)=\mathfrak{k} \otimes_{\mathcal{O}_{\mathfrak{G} / \mathfrak{H}, e}} \mathcal{M}_{e} \text {. }
$$

Let $(\operatorname{Dist}(\mathfrak{G}), \mathfrak{H})$ Mod be the category of Harish-Chandra (Dist $(\mathfrak{G}), \mathfrak{H})$-modules $[\mathbf{H} 87,4.3 .8]$, that are the same as $\mathfrak{k}$-linear spaces equipped with a structure of compatible $\mathfrak{G}_{r} \mathfrak{H}$-modules, $r \in \mathbb{N}$. We can generalize [H87, 4.3.7/9] as in characteristic 0 [Ka, Th. 4.10.2] to:

Theorem. The functor $\mathcal{L}_{\mathfrak{G} / \mathfrak{H}}$ induces upon restriction an equivalence of categories

$$
(\operatorname{Dist}(\mathfrak{G}), \mathfrak{H}) \operatorname{Mod} \rightarrow\left(\mathfrak{G}, \mathcal{D}_{\mathfrak{G} / \mathfrak{H}}\right) \mathbf{q q} \mathbf{c} .
$$

In particular,$\quad \mathcal{L}_{\mathfrak{G} / \mathfrak{H}}\left(\operatorname{Dist}(\mathfrak{G}) \otimes_{\operatorname{Dist}(\mathfrak{H})} \mathfrak{k}\right) \simeq \mathcal{D}_{\mathfrak{G} / \mathfrak{H}} \quad$ in $\left(\mathfrak{G}, \mathcal{D}_{\mathfrak{G} / \mathfrak{H}}\right) \mathbf{q q} \mathbf{c}$.

Proof. Put $\mathfrak{X}=\mathfrak{G} / \mathfrak{H}, \mathcal{L}=\mathcal{L}_{\mathfrak{G} / \mathfrak{H}}$, and denote the $\mathfrak{G}$-action on $\mathfrak{X}$ by $\mathfrak{a}$. Let $\mathfrak{a}_{r}=\mathfrak{a}^{(r)} \circ\left(\mathfrak{F}_{\mathfrak{G}}^{r} \times \mathfrak{X}^{(r)}\right)$ and $\mathfrak{p}_{r}=\mathfrak{p}_{\mathfrak{X}}^{(r)} \circ\left(\mathfrak{F}_{\mathfrak{G}}^{r} \times \mathfrak{X}^{(r)}\right): \mathfrak{G} \times \mathfrak{X}^{(r)} \rightarrow \mathfrak{X}^{(r)}$. We will identify $\mathfrak{X}^{(r)}$ with $\mathfrak{G} / \mathfrak{G}_{r} \mathfrak{H}$ via the commutative diagram

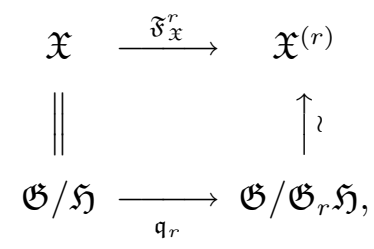

where $\mathfrak{q}_{r}: \mathfrak{G} / \mathfrak{H} \rightarrow \mathfrak{G} / \mathfrak{G}_{r} \mathfrak{H}$ is the quotient morphism. 
If $M \in(\operatorname{Dist}(\mathfrak{G}), \mathfrak{H})$ Mod, let $\phi_{r}: \mathfrak{a}_{r}^{*} \mathcal{L}_{\mathfrak{G} / \mathfrak{G}_{r} \mathfrak{H}}(M) \rightarrow \mathfrak{p}_{r}^{*} \mathcal{L}_{\mathfrak{G} / \mathfrak{G}_{r} \mathfrak{H}}(M)$ be the structure morphism of $\mathcal{L}_{\mathfrak{G} / \mathfrak{G}_{r} \mathfrak{H}}(M)$ in $\left(\mathfrak{G}, \mathfrak{X}^{(r)}\right) \mathbf{q c}$. Then $\left(\mathcal{L}_{\mathfrak{G} / \mathfrak{G}_{r} \mathfrak{H}}(M), \phi_{r}\right)_{r} \in$ $\left(\mathfrak{G}, \mathfrak{X}^{\infty}\right) \mathbf{q q} \mathbf{c}$.

On the other hand, if $\mathcal{M} \in\left(\mathfrak{G}, \mathcal{D}_{\mathfrak{X}}\right)$ qqc with a structure morphism $\phi$, then $\left(\mathcal{M}^{(r)}, \phi_{r}\right)_{r} \in\left(\mathfrak{G}, \mathfrak{X}^{\infty}\right) \mathbf{q c}$ by (3.13). Hence for each $r \in \mathbb{N}$ one has in $\operatorname{Mod}_{\mathfrak{k}}$

$$
\begin{aligned}
\mathfrak{k} \otimes_{\mathcal{O}_{\mathfrak{X}, e}} \mathcal{M}_{e} & \simeq \mathfrak{k} \otimes_{\mathcal{O}_{\mathfrak{X}, e}} \mathcal{O}_{\mathfrak{X}, e} \otimes_{\mathcal{O}_{\mathfrak{X}, e}^{(r)}}\left(\mathcal{M}^{(r)}\right)_{e} \simeq \mathfrak{k} \otimes_{\mathcal{O}_{\mathfrak{X}, e}^{(r)}}\left(\mathcal{M}^{(r)}\right)_{e} \\
& \simeq \mathfrak{k} \otimes_{\mathcal{O}_{\mathfrak{X}, e}^{(r)}} \mathcal{O}_{\mathfrak{X}, e}^{(r)} \otimes_{\mathcal{O}_{\mathfrak{X}, e}^{(r+1)}}\left(\mathcal{M}^{(r+1)}\right)_{e}
\end{aligned}
$$

We may regard $\left(\mathcal{M}^{(r)}, \phi_{r}\right)_{r} \in\left(\mathfrak{G}, \mathfrak{G} / \mathfrak{G}_{r} \mathfrak{H}\right) \mathbf{q c}$. If $\bar{\varepsilon}_{r}=\mathfrak{q}_{r} \circ \varepsilon_{\mathfrak{G}}: \mathfrak{e} \rightarrow \mathfrak{G} / \mathfrak{G} \mathfrak{H}_{r} \mathfrak{H}$ and if we let $\mathfrak{G}_{r} \mathfrak{H}$ act on $\mathfrak{e}$ trivially, then $\left(\bar{\varepsilon}_{r}\right)^{*}\left(\mathcal{M}^{(r)}\right) \simeq \mathfrak{k} \otimes_{\mathcal{O}_{\mathfrak{X}, e}^{(r)}}\left(\mathcal{M}^{(r)}\right)_{e}$ admits a structure of $\mathfrak{G}_{r} \mathfrak{H}$-module as $\bar{\varepsilon}_{r}$ is $\mathfrak{G}_{r} \mathfrak{H}$-equivariant. Moreover, $\bar{\varepsilon}_{r+1}=\mathfrak{q}_{r, r+1} \circ \bar{\varepsilon}_{r}$ with $\mathfrak{q}_{r, r+1}: \mathfrak{G} / \mathfrak{G}_{r} \mathfrak{H} \rightarrow \mathfrak{G} / \mathfrak{G}_{r+1} \mathfrak{H}$ the quotient morphism, hence

$$
\left(\bar{\varepsilon}_{r+1}\right)^{*}\left(\mathcal{M}^{(r+1)}\right) \simeq\left(\bar{\varepsilon}_{r}\right)^{*}\left(\mathcal{M}^{(r)}\right) \text { in } \mathfrak{G}_{r} \mathfrak{H} \mathbf{M o d}
$$

Then $\mathcal{M}(e)=\mathfrak{k} \otimes_{\mathcal{O}_{\mathfrak{x}, e}} \mathcal{M}_{e}$ comes equipped with a structure of $\mathfrak{G}_{r} \mathfrak{H}$-module compatibly with respect to $r \in \mathbb{N}$, and the first assertion follows.

To see the second assertion, recall that

$$
\operatorname{Dist}(\mathfrak{G}) \otimes_{\operatorname{Dist}(\mathfrak{H})} \mathfrak{k} \simeq \underset{r}{\varliminf_{i m}} \operatorname{Dist}\left(\mathfrak{G}_{r}\right) \otimes_{\operatorname{Dist}\left(\mathfrak{H}_{r}\right)} \mathfrak{k} \quad \text { in } \operatorname{Dist}(\mathfrak{G}) \operatorname{Mod}
$$

and that (cf. [J, I.8.15, 8.20])

$$
\operatorname{Dist}\left(\mathfrak{G}_{r}\right) \otimes_{\operatorname{Dist}\left(\mathfrak{H}_{r}\right)} \mathfrak{k} \simeq\left(\operatorname{ind}_{\mathfrak{H}}^{\mathfrak{G}_{r} \mathfrak{H}} \mathfrak{k}\right)^{*} \quad \text { in } \mathfrak{G}_{r} \mathfrak{H} \operatorname{Mod}
$$

We have isomorphisms in $\operatorname{Mod}_{\mathfrak{X}(r)}$

$$
\begin{aligned}
\mathcal{D}_{\mathfrak{X}, r} & \simeq \operatorname{Mod}_{\mathcal{O}_{\mathfrak{X}}^{(r)}}\left(\mathcal{O}_{\mathfrak{X}}, \mathcal{O}_{\mathfrak{X}}\right) \simeq \operatorname{Mod}_{\mathfrak{X}}\left(\left(\mathfrak{F}_{\mathfrak{X}}^{r}\right)^{*}\left(\mathfrak{F}_{\mathfrak{X}}^{r}\right)_{*} \mathcal{O}_{\mathfrak{X}}, \mathcal{O}_{\mathfrak{X}}\right) \\
& \simeq \operatorname{Mod}_{\mathfrak{X}}\left(\mathfrak{q}_{r}^{*}\left(\mathfrak{q}_{r}\right)_{*} \mathcal{O}_{\mathfrak{X}}, \mathcal{O}_{\mathfrak{X}}\right) \simeq \mathcal{M o d} \operatorname{Mo}_{\mathfrak{X}}\left(\mathcal{L}\left(\operatorname{ind}_{\mathfrak{H}}^{\mathfrak{G}_{r} \mathfrak{H}} \mathfrak{k}\right), \mathcal{O}_{\mathfrak{X}}\right) \\
& \simeq \mathcal{L}\left(\left(\operatorname{ind}_{\mathfrak{H}}^{\mathfrak{G}_{r} \mathfrak{H}} \mathfrak{k}\right)^{*}\right) .
\end{aligned}
$$

It is enough to show that the composite isomorphism of (3) belongs to $\mathcal{D}_{\mathfrak{X}, r}$ Mod and also to $(\mathfrak{G}, \mathfrak{X}) \mathbf{q c}$. The questions being local, let $\mathfrak{U}$ be an affine open of $\mathfrak{G} / \mathfrak{G}_{r} \mathfrak{H}, \mathfrak{V}=\mathfrak{q}_{r}^{-1} \mathfrak{U}$ and $\mathfrak{U}^{\prime}=\pi_{r}^{-1} \mathfrak{U}$ with $\pi_{r}: \mathfrak{G} \rightarrow \mathfrak{G} / \mathfrak{G}_{r} \mathfrak{H}$ the quotient morphism. Both $\mathfrak{U}^{\prime}$ and $\mathfrak{V}$ remain affine. Recall an isomorphism (cf. [J, I.5.18.5])

$$
\begin{aligned}
\Gamma\left(\mathfrak{U},\left(\mathfrak{q}_{r}\right)_{*} \mathcal{O}_{\mathfrak{X}}\right)=\mathfrak{k}\left[\mathfrak{U}^{\prime}\right]^{\mathfrak{H}} \rightarrow \operatorname{Sch}_{\mathfrak{k}}\left(\mathfrak{U}^{\prime}, \operatorname{ind}_{\mathfrak{H}}^{\mathfrak{G}_{r} \mathfrak{H}}(\mathfrak{k})\right)^{\mathfrak{G}_{r} \mathfrak{H}} & \\
& =\Gamma\left(\mathfrak{U}, \mathcal{L}_{\mathfrak{G} / \mathfrak{G}_{r} \mathfrak{H}}\left(\operatorname{ind}_{\mathfrak{H}}^{\mathfrak{G}_{r} \mathfrak{H}^{\mathfrak{H}}}(\mathfrak{k})\right)\right)
\end{aligned}
$$


via $a \mapsto \hat{a}$ such that

$$
\hat{a}(x)=a(x ?) \in \mathbf{S c h}_{\mathfrak{k}}\left(\mathfrak{G}_{r} \mathfrak{H}, \mathfrak{k}\right)^{\mathfrak{H}}=\operatorname{ind}_{\mathfrak{H}}^{\mathfrak{H}_{r} \mathfrak{H}}(\mathfrak{k}), \quad x \in \mathfrak{U}^{\prime},
$$

and another (cf. $[\mathbf{K 9 5}, 1.8]$ ) for each $\mathfrak{G}_{r} \mathfrak{H}$-module $Q$

$$
\begin{aligned}
\Gamma\left(\mathfrak{V}, \mathfrak{q}_{r}^{*} \mathcal{L}_{\mathfrak{G} / \mathfrak{G}_{r} \mathfrak{H}}(Q)\right) & =\mathfrak{k}\left[\mathfrak{U}^{\prime}\right]^{\mathfrak{H}} \otimes_{\mathfrak{k}\left[\mathfrak{U}^{\prime}\right]^{\mathfrak{G}_{r} \mathfrak{H}}} \mathbf{S c h}_{\mathfrak{k}}\left(\mathfrak{U}^{\prime}, Q\right)^{\mathfrak{G}_{r} \mathfrak{H}} \\
& \rightarrow \operatorname{Sch}_{\mathfrak{k}}\left(\mathfrak{U}^{\prime}, Q\right)^{\mathfrak{H}}=\Gamma(\mathfrak{V}, \mathcal{L}(Q))
\end{aligned}
$$

via $a \otimes f \mapsto a f$. Hence on $\mathfrak{V}$ the composite (3) sends

$$
\theta \in \operatorname{Sch}_{\mathfrak{k}}\left(\mathfrak{U}^{\prime}, \operatorname{ind}_{\mathfrak{H}}^{\mathfrak{G}_{r} \mathfrak{H}}(\mathfrak{k})^{*}\right)^{\mathfrak{H}}=\mathcal{L}\left(\left(\operatorname{ind}_{\mathfrak{H}}^{\mathfrak{G}_{r} \mathfrak{H}} \mathfrak{k}\right)^{*}\right)(\mathfrak{V})
$$

to $\tilde{\theta}=\langle\theta, \hat{?}\rangle \in D_{r}\left(\mathfrak{k}\left[\mathfrak{U}^{\prime}\right]^{\mathfrak{H}}\right)$ with $\langle\theta, \hat{a}\rangle \in \mathfrak{k}\left[\mathfrak{U}^{\prime}\right]^{\mathfrak{H}}, a \in \mathfrak{k}\left[\mathfrak{U}^{\prime}\right]^{\mathfrak{H}}$, such that

$$
\langle\theta, \hat{a}\rangle(x)=\langle\theta(x), \hat{a}(x)\rangle, \quad x \in \mathfrak{U}^{\prime} .
$$

The $\mathcal{D}_{\mathfrak{X}, r}$-module structure on $\mathcal{L}\left(\left(\operatorname{ind}_{\mathfrak{H}}^{\mathfrak{G}_{h} \mathfrak{H}} \mathfrak{k}\right)^{*}\right)$ is given on $\mathfrak{V}$ by $a \otimes f \mapsto \delta(a) \otimes f, \quad a \in \mathfrak{k}\left[\mathfrak{U}^{\prime}\right]^{\mathfrak{H}}, f \in \mathbf{S c h}_{\mathfrak{k}}\left(\mathfrak{U}^{\prime},\left(\operatorname{ind}_{\mathfrak{H}}^{\mathfrak{H}_{r} \mathfrak{H}} \mathfrak{k}\right)^{*}\right)^{\mathfrak{G}_{r} \mathfrak{H}}, \delta \in D_{r}\left(\mathfrak{k}\left[\mathfrak{U}^{\prime}\right]^{\mathfrak{H}}\right)$ under the identification (5), hence by $a f \mapsto \delta(a) f$. Then we must show

$$
(\delta(a) f)^{\sim}=\delta \circ \widetilde{a f} \quad \text { in } D_{r}\left(\mathfrak{k}\left[\mathfrak{U}^{\prime}\right]^{\mathfrak{H}}\right) .
$$

If $b \in \mathfrak{k}\left[\mathfrak{U}^{\prime}\right]^{\mathfrak{H}}$ and $x \in \mathfrak{U}^{\prime}$, then

$$
\begin{aligned}
\left\{(\delta(a) f)^{\sim}(b)\right\}(x) & =\langle\delta(a) f, \hat{b}\rangle(x)=\langle(\delta(a) f)(x), \hat{b}(x)\rangle \\
& =\langle(\delta(a))(x) f(x), b(x ?)\rangle=(\delta(a))(x)\langle f(x), b(x ?)\rangle
\end{aligned}
$$

while

$$
\begin{aligned}
\{(\delta \circ \widetilde{a f})(b)\}(x) & =\{\delta(a\langle f, \hat{b}\rangle)\}(x) \\
& =\{\delta(a)\langle f, \hat{b}\rangle\}(x) \quad \text { as }\langle f, \hat{b}\rangle \in \mathfrak{k}\left[\mathfrak{U}^{\prime}\right]^{\mathfrak{G}_{r} \mathfrak{H}} \simeq\left(\mathfrak{k}\left[\mathfrak{U}^{\prime}\right]^{\mathfrak{H}}\right)^{(r)} \\
& =(\delta(a))(x)\langle f(x), \hat{b}(x)\rangle,
\end{aligned}
$$

hence (7), and the composite (3) belongs to $\mathcal{D}_{\mathfrak{X}, r}$ Mod.

To see next that the composite belongs to $(\mathfrak{G}, \mathfrak{X}) \mathbf{q c}$, it suffices to show by (3.7) that the composite is $\operatorname{Dist}\left(\mathfrak{G}_{s}\right)^{\mathrm{op}}$-equivariant on $\mathfrak{V}$ for each $s \in \mathbb{N}$, i.e., the composite yields

$$
\mathcal{L}\left(\left(\operatorname{ind}_{\mathfrak{H}}^{\mathfrak{G}_{H} \mathfrak{H}} \mathfrak{k}\right)^{*}\right)(\mathfrak{V}) \simeq \mathcal{D}_{\mathfrak{X}, r}(\mathfrak{V}) \quad \text { in } \mathfrak{G}_{s}^{\text {op }} \text { Mod }
$$

The assertion will follow from:

\subsection{5 .}

Lemma. Let $M \in \mathfrak{H}$ Mod.

(i) $\quad \mathcal{L}_{\mathfrak{G} / \mathfrak{G}_{r} \mathfrak{H}}\left(\operatorname{ind}_{\mathfrak{H}}^{\mathfrak{G}_{r} \mathfrak{H}} M\right) \simeq\left(\mathfrak{q}_{r}\right)_{*} \mathcal{L}_{\mathfrak{G} / \mathfrak{H}}(M) \quad$ in $\left(\mathfrak{G}, \mathfrak{G} / \mathfrak{G}_{r} \mathfrak{H}\right) \mathbf{q} \mathbf{c}$ 
(ii) $\quad \mathcal{L}_{\mathfrak{G} / \mathfrak{H}}\left(\operatorname{ind}_{\mathfrak{H}}^{\mathfrak{G}_{r} \mathfrak{H}} M\right) \simeq \mathfrak{q}_{r}^{*}\left(\mathfrak{q}_{r}\right)_{*} \mathcal{L}_{\mathfrak{G} / \mathfrak{H}}(M) \quad$ in $(\mathfrak{G}, \mathfrak{G} / \mathfrak{H}) \mathbf{q c}$

Proof. We carry over the notations of the previous section. By taking direct limit we may assume $M$ is finite dimensional. Then the question being local, we have by (3.7) only to show for all $s \in \mathbb{N}$

$$
\Gamma\left(\mathfrak{U}, \mathcal{L}_{\mathfrak{G} / \mathfrak{G}_{r} \mathfrak{H}}\left(\operatorname{ind}_{\mathfrak{H}}^{\mathfrak{G}_{r} \mathfrak{H}}(M)\right)\right) \simeq \Gamma\left(\mathfrak{U},\left(\mathfrak{q}_{r}\right)_{*} \mathcal{L}(M)\right) \quad \text { in } \mathfrak{G}_{s}^{\text {op }} \mathbf{M o d}
$$

and

$$
\Gamma\left(\mathfrak{V}, \mathcal{L}\left(\operatorname{ind}_{\mathfrak{H}}^{\mathfrak{G}_{r} \mathfrak{H}}(M)\right)\right) \simeq \Gamma\left(\mathfrak{V}, \mathfrak{q}_{r}^{*}\left(\mathfrak{q}_{r}\right)_{*} \mathcal{L}(M)\right) \quad \text { in } \mathfrak{G}_{s}^{\text {op }} \mathbf{M o d}
$$

But in (1) we have an isomorphism in $\mathfrak{G}_{s}^{\text {op }} \mathbf{M o d}$

$$
\begin{aligned}
\Gamma\left(\mathfrak{U}, \mathcal{L}_{\mathfrak{G} / \mathfrak{G}_{r} \mathfrak{H}}\left(\operatorname{ind}_{\mathfrak{H}}^{\mathfrak{G}_{r} \mathfrak{H}}(M)\right)\right) & =\operatorname{Sch}_{\mathfrak{k}}\left(\mathfrak{U}^{\prime}, \operatorname{ind}_{\mathfrak{H}}^{\mathfrak{G}_{r} \mathfrak{H}}(M)\right)^{\mathfrak{G}_{r} \mathfrak{H}} \\
& \longrightarrow \operatorname{Sch}_{\mathfrak{k}}\left(\mathfrak{U}^{\prime}, M\right)^{\mathfrak{H}}=\Gamma\left(\mathfrak{U},\left(\mathfrak{q}_{r}\right)_{*} \mathcal{L}(M)\right)
\end{aligned}
$$

via $f \mapsto \mathrm{ev}_{M} \circ f$ with $\operatorname{ev}_{M}: \operatorname{ind}_{\mathfrak{H}}^{\mathfrak{G}_{r} \mathfrak{H}}(M) \rightarrow M$ the evaluation at $e$. Likewise (2) using (1).

\section{$\S 4$.}

In this section $\mathfrak{X}$ will denote a flag variety $G / B$ with $G$ a simply connected semisimple $\mathfrak{k}$-group and $B$ a Borel subgroup of $G$. Let $T$ be a maximal torus of $B, W=\mathrm{N}_{G}(T) / T$ the Weyl group, $B^{+}$the Borel subgroup of $G$ opposite to $B$, and $U^{+}$the unipotent radical of $B^{+}$. For all other unexplained standard notations we refer to $[\mathbf{J}]$.

4.1. Fix $w \in W$. In $\mathfrak{X}$ let $\mathfrak{X}_{w}=U^{+} w B / B, \overline{\mathfrak{X}_{w}}$ the closure of $\mathfrak{X}_{w}, \mathfrak{d} \mathfrak{X}_{w}=$ $\overline{\mathfrak{X}_{w}} \backslash \mathfrak{X}_{w}, \mathfrak{N}_{w}=w U^{+} B / B, \mathfrak{i}_{w}: \mathfrak{X}_{w} \hookrightarrow \mathfrak{N}_{w}$, and $\mathfrak{j}_{w}: \mathfrak{N}_{w} \hookrightarrow \mathfrak{X}$. Then $\mathfrak{N}_{w}$ is an affne open of $\mathfrak{X}$, and $\mathfrak{X}_{w}=\overline{\mathfrak{X}_{w}} \cap \mathfrak{N}_{w}$ of codimension $\ell(w)$, the length of $w$, in $\mathfrak{N}_{w}$. If ${ }^{w} U^{+}=w U^{+} w^{-1}$ and $U_{w}^{+}={ }^{w} U^{+} \cap U^{+}$, there is a commutative diagram of $\mathfrak{k}$-varieties

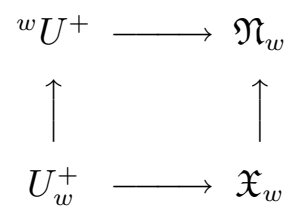

with the vertical arrows being inclusions and the horizontal ones ivertible given by $x \mapsto x w B$. Let $\mathcal{I}_{w}$ be the ideal sheaf of $\mathfrak{X}_{w}$ in $\mathfrak{N}_{w}$. We have as in characteristic 0:

Proposition. Let $\ell=\ell(w)$ and $B_{w}^{+}=\left(w B^{+} w^{-1}\right) \cap B^{+}$.

(i) $\quad$ On $\mathrm{D}^{b}\left(\mathcal{D}_{\mathfrak{X}} \mathbf{q} \mathbf{c}\right) \quad \mathrm{R} \Gamma_{\overline{\mathfrak{X}_{w}} / \mathfrak{o \mathfrak { X } _ { w }}} \simeq\left(\mathfrak{j}_{w}\right)_{0} \circ \mathrm{R} \Gamma_{\mathfrak{X}_{w}} \circ \mathfrak{j}_{w}^{-1} \simeq \int_{\mathfrak{j}_{w} \circ \mathfrak{i}_{w}} \mathrm{~L}\left(\mathfrak{j}_{w} \circ \mathfrak{i}_{w}\right)^{0}[-\ell]$. In particular, if $\mathcal{M} \in \mathrm{D}^{b}\left(\left(B^{+}, \mathcal{D}_{\mathfrak{X}}\right) \mathbf{q} \mathbf{c}\right), \mathrm{R} \Gamma_{\overline{\mathfrak{X}_{w}} / \mathfrak{o} \mathfrak{X}_{w}}\left(\mathcal{M}^{\cdot}\right) \simeq \int_{\mathfrak{j}_{w} \circ \mathfrak{i}_{w}} \mathrm{~L}\left(\mathfrak{j}_{w} \circ\right.$ $\left.\mathfrak{i}_{w}\right)^{0}\left(\mathcal{M}^{\cdot}\right)[-\ell]$ in $\mathrm{D}^{b}\left(\left(B^{+}, \mathcal{D}_{\mathfrak{X}}\right) \mathbf{q} \mathbf{c}\right)$. 
(ii) $\quad$ On $\mathrm{D}^{b}\left(\mathbf{A b}_{\mathfrak{X}}\right) \quad \mathrm{R} \Gamma_{\mathfrak{d} \mathfrak{X}_{w}} \circ \mathrm{R} \Gamma_{\overline{\mathfrak{X}_{w}} / \mathfrak{d} \mathfrak{X}_{w}}=0$.

(iii) $\operatorname{In}\left(B^{+}, \mathcal{D}_{\mathfrak{X}}\right) \mathbf{q c} \quad \forall s \in \mathbb{N}$,

$$
\mathcal{H}_{\overline{\mathfrak{X}}_{w} / \mathfrak{o} \mathfrak{X}_{w}}\left(\mathcal{O}_{\mathfrak{X}}\right) \simeq \begin{cases}\int_{\mathfrak{j}_{w} \circ \mathfrak{i}_{w}}^{0} \mathcal{O}_{\mathfrak{X}_{w}} & \text { if } s=\ell \\ 0 & \text { otherwise. }\end{cases}
$$

(iv) (cf. $\left[\mathbf{B} \varnothing\right.$, Prop. 4.7].) In $\left(B_{w}^{+}, \mathcal{D}_{\mathfrak{X}}\right) \mathbf{q c}$

$$
\left.\mathcal{H}_{\mathfrak{X}_{w} / \mathfrak{d} \mathfrak{X}_{w}}^{\ell}\left(\mathcal{O}_{\mathfrak{X}}\right)\right|_{\mathfrak{N}_{w}} \simeq \mathcal{H}_{\mathfrak{X}_{w}}^{\ell}\left(\mathcal{O}_{\mathfrak{N}_{w}}\right) \simeq \mathcal{B}_{\mathfrak{X}_{w} \mid \mathfrak{N}_{w}},
$$

and in $\left(B_{w}^{+}, D\left(\mathfrak{N}_{w}\right)\right)$ Mod

$$
\begin{aligned}
\mathcal{B}_{\mathfrak{X}_{w} \mid \mathfrak{N}_{w}}\left(\mathfrak{N}_{w}\right) & \simeq\left\{D\left(\mathfrak{N}_{w}\right) /\left(D\left(\mathfrak{N}_{w}\right) \operatorname{Dist}^{+}\left(U_{w}^{+}\right)+D\left(\mathfrak{N}_{w}\right) \mathcal{I}_{w}\left(\mathfrak{N}_{w}\right)\right)\right\} \otimes \mathfrak{k}_{w \cdot 0} \\
& \simeq \mathfrak{k}\left[U_{w}^{+}\right] \otimes \operatorname{Dist}\left(U_{w}^{-}\right) \otimes \mathfrak{k}_{w \cdot 0} .
\end{aligned}
$$

In particular,

$$
\operatorname{ch~} \mathrm{H}_{\mathfrak{X}_{w} / \mathfrak{O} \mathfrak{X}_{w}}\left(\mathfrak{X}, \mathcal{O}_{\mathfrak{X}}\right)=e(w \cdot 0) \prod_{\alpha \in R^{+}} \frac{1}{1-e(-\alpha)},
$$

where ch is the formal character of the T-module in question obtained from the $T^{\mathrm{op}}$-module by inversion on $T$.

(v) (cf. [Bø, Prop. 4.7].) If $\mathcal{L}(w)=\mathcal{D}_{\mathfrak{X}}\left\{\mathrm{H}_{\mathfrak{X}_{w} / \mathfrak{o} \mathfrak{X}_{w}}\left(\mathfrak{X}, \mathcal{O}_{\mathfrak{X}}\right)_{w \cdot 0}\right\}$, then

$$
\begin{aligned}
& \operatorname{supp}\left(\mathcal{H}_{\overline{\mathfrak{X}_{w}} / \mathfrak{d} \mathfrak{X}_{w}}^{\ell}\left(\mathcal{O}_{\mathfrak{X}}\right)\right)=\overline{\mathfrak{X}_{w}}=\operatorname{supp}(\mathcal{L}(w)) \quad \text { and } \\
& \operatorname{supp}\left(\mathcal{H}_{\overline{\mathfrak{X}_{w}} / \mathfrak{d} \mathfrak{X}_{w}}\left(\mathcal{O}_{\mathfrak{X}}\right) / \mathcal{L}(w)\right) \subseteq \mathfrak{d} \mathfrak{X}_{w} .
\end{aligned}
$$

Proof. Let $\mathfrak{j}_{\mathfrak{d}}: \mathfrak{N}_{w} \rightarrow \mathfrak{X} \backslash \mathfrak{d} \mathfrak{X}_{w}$ and $\mathfrak{j}_{\mathfrak{d}}^{\prime}: \mathfrak{X} \backslash \mathfrak{d} \mathfrak{X}_{w} \rightarrow \mathfrak{X}$ be two inclusions.

(i) On flasques of $\mathbf{A} \mathbf{b}_{\mathfrak{X}}$

$$
\begin{aligned}
\Gamma_{\overline{\mathfrak{X}_{w}} / \mathfrak{o} \mathfrak{X}_{w}} & \simeq\left(\mathfrak{j}_{\mathfrak{d}}^{\prime}\right)_{*} \circ \Gamma_{\mathfrak{X}_{w}} \circ\left(\mathfrak{j}_{\mathfrak{d}}^{\prime}\right)^{-1} \quad \text { by }[\mathbf{K e}, 8.3] \\
& \simeq\left(\mathfrak{j}_{w}\right)_{*} \circ \Gamma_{\mathfrak{X}_{w}} \circ\left(\mathfrak{j}_{w}\right)^{-1} \quad \text { by excision }[\mathbf{K e}, 7.9],
\end{aligned}
$$

hence on $\mathrm{D}^{b}\left(\mathcal{D}_{\mathfrak{x}} \mathbf{q} \mathbf{c}\right)$

$$
\begin{aligned}
& \mathrm{R} \Gamma_{\overline{\mathfrak{X}_{w}} / \circ \mathfrak{X}_{w}} \\
& \simeq \mathrm{R}\left(\mathfrak{j}_{w}\right)_{0} \circ \mathrm{R} \Gamma_{\mathfrak{X}_{w}} \circ \mathrm{R}\left(\mathfrak{j}_{w}^{-1}\right) \text { as both } \Gamma_{\mathfrak{X}_{w}} \text { and } \mathfrak{j}_{w}^{-1} \text { send flasques to flasques } \\
& \simeq\left(\mathfrak{j}_{w}\right)_{0} \circ \mathrm{R} \Gamma_{\mathfrak{X}_{w}} \circ \mathfrak{j}_{w}^{-1} \text { by Serre's theorem as } \mathfrak{j}_{w} \text { is affine } \\
& \simeq\left(\mathfrak{j}_{w}\right)_{0} \circ \int_{\mathfrak{i}_{w}} \mathrm{~L}\left(\mathfrak{i}_{w}^{0}\right)[-\ell] \circ \mathfrak{j}_{w}^{-1} \text { by }(2.11) \\
& \simeq \int_{\mathfrak{j}_{w} \circ \mathfrak{i}_{w}} \mathrm{~L}\left(\mathfrak{j}_{w} \circ \mathfrak{i}_{w}\right)^{0}[-\ell] \quad \text { by }(2.6) .
\end{aligned}
$$


(ii) By (i), as $\left(\mathfrak{j}_{\mathfrak{d}}^{\prime}\right)_{*} \circ \Gamma_{\mathfrak{X}_{w}} \circ\left(\mathfrak{j}_{\mathfrak{d}}^{\prime}\right)^{-1}$ sends flasques to flasques,

$$
\mathrm{R} \Gamma_{\mathfrak{o} \mathfrak{X}_{w}} \circ \mathrm{R} \Gamma_{\overline{\mathfrak{X}_{w}} / \mathfrak{o x}} \simeq \mathrm{R}\left(\Gamma_{\mathfrak{o} \mathfrak{X}_{w}} \circ\left(\mathfrak{j}_{\mathfrak{d}}^{\prime}\right)_{*} \circ \Gamma_{\mathfrak{X}_{w}} \circ\left(\mathfrak{j}_{\mathfrak{o}}^{\prime}\right)^{-1}\right) .
$$

As $\Gamma_{\mathfrak{d} \mathfrak{X}_{w}} \circ\left(\mathfrak{j}_{\mathfrak{d}}^{\prime}\right)_{*} \circ \Gamma_{\mathfrak{X}_{w}} \circ\left(\mathfrak{j}_{\mathfrak{d}}^{\prime}\right)^{-1}$ is left exact and as any sheaf of abelian groups can be imbedded in a flasque sheaf, it is enough to show that $\Gamma_{\mathfrak{o} \mathfrak{X}_{w}} \circ\left(\mathfrak{j}_{\mathfrak{d}}^{\prime}\right)_{*} \circ$ $\Gamma_{\mathfrak{X}_{w}} \circ\left(\mathfrak{j}_{\mathfrak{d}}^{\prime}\right)^{-1}=0$ on flasques. But on flasques the LHS is $\Gamma_{\mathfrak{X}_{w}} \circ \Gamma_{\overline{\mathfrak{X}_{w}} / \mathfrak{o} \mathfrak{X}_{w}}$ by (1). If $\mathcal{F}$ is a flasque sheaf, the short exact sequence

$$
0 \rightarrow \Gamma_{\mathfrak{d} \mathfrak{X}_{w}}(\mathcal{F}) \rightarrow \Gamma_{\overline{\mathfrak{X}_{w}}}(\mathcal{F}) \rightarrow \Gamma_{\overline{\mathfrak{X}_{w}} / \mathfrak{o X}}(\mathcal{F}) \rightarrow 0
$$

remains exact after applying $\Gamma_{\mathfrak{d} \mathfrak{X}_{w}}$ as $\Gamma_{\mathfrak{d} \mathfrak{X}_{w}}(\mathcal{F})$ is flasque. Then

$$
\Gamma_{\mathfrak{d} \mathfrak{X}_{w}} \circ \Gamma_{\overline{\mathfrak{X}_{w}} / \mathfrak{d} \mathfrak{X}_{w}}(\mathcal{F}) \simeq \Gamma_{\mathfrak{d} \mathfrak{X}_{w}}\left(\Gamma_{\overline{\mathfrak{X}_{w}}}(\mathcal{F})\right) / \Gamma_{\mathfrak{d} \mathfrak{X}_{w}}\left(\Gamma_{\mathfrak{d} \mathfrak{X}_{w}}(\mathcal{F})\right)=0 .
$$

(iii) As $\mathfrak{j}_{w}$ is affine and as $\mathfrak{i}_{w}$ is a closed immersion, both $\left(\mathfrak{j}_{w}\right)_{0}$ and $\left(\mathfrak{i}_{w}\right)_{0}$ are exact, hence $\int_{\mathfrak{j}_{w} \circ \mathfrak{i}_{w}} \simeq\left(\mathfrak{j}_{w}\right)_{0} \circ\left(\mathfrak{i}_{w}\right)_{0}\left(\mathcal{D}_{\mathfrak{i}_{w} \leftarrow \otimes_{\mathcal{D}_{\mathfrak{x}_{w}}}}\right.$ ?) is exact. Then

$$
\mathcal{H}_{\mathfrak{X}_{w} / \mathfrak{o} \mathfrak{X}_{w}}^{s}\left(\mathcal{O}_{\mathfrak{X}}\right) \simeq \int_{\mathfrak{j}_{w} \circ \mathfrak{i}_{w}} L^{s-\ell}\left(\left(\mathfrak{j}_{w} \circ \mathfrak{i}_{w}\right)^{0}\right)\left(\mathcal{O}_{\mathfrak{X}}\right) \simeq \int_{\mathfrak{j}_{w} \circ \mathfrak{i}_{w}}^{0} L^{s-\ell}\left(\left(\mathfrak{j}_{w} \circ \mathfrak{i}_{w}\right)^{0}\right)\left(\mathcal{O}_{\mathfrak{X}}\right) .
$$

By (2.13)

$$
\mathrm{L}^{s-\ell}\left(\left(\mathfrak{j}_{w} \circ \mathfrak{i}_{w}\right)^{0}\right)\left(\mathcal{O}_{\mathfrak{X}}\right) \simeq \mathrm{L}^{s-\ell}\left(\mathfrak{i}_{w}^{0}\right)\left(\mathcal{O}_{\mathfrak{N}_{w}}\right) \simeq \begin{cases}\mathcal{O}_{\mathfrak{X}_{w}} & \text { if } s=\ell \\ 0 & \text { otherwise }\end{cases}
$$

(iv) By (i), (iii) and as $\mathbf{j}_{w}$ is affine, one has in $\left(B_{w}^{+}, \mathcal{D}_{\mathfrak{X}}\right) \mathbf{q c}$

$$
\mathcal{H}_{\overline{\mathfrak{X}_{w}} / \mathfrak{\varrho} \mathfrak{X}_{w}}\left(\mathcal{O}_{\mathfrak{X}}\right) \simeq\left(\mathfrak{j}_{w}\right)_{0} \circ \mathcal{H}_{\mathfrak{X}_{w}}^{\ell}\left(\mathcal{O}_{\mathfrak{X}}\right) \simeq\left(\mathfrak{j}_{w}\right)_{0} \circ \int_{\mathfrak{i}_{w}}^{0} \mathcal{O}_{\mathfrak{X}_{w}} \simeq\left(\mathfrak{j}_{w}\right)_{0}\left(\mathcal{B}_{\mathfrak{X}_{w} \mid \mathfrak{N}_{w}}\right) .
$$

Identifying $D\left(\mathfrak{N}_{w}\right)$ with $D\left(U_{w}^{+}\right) \otimes D\left(U_{w}^{-}\right) \simeq \mathfrak{k}\left[U_{w}^{+}\right] \otimes \operatorname{Dist}\left(U_{w}^{+}\right) \otimes \mathfrak{k}\left[U_{w}^{-}\right] \otimes$ $\operatorname{Dist}\left(U_{w}^{-}\right)$one has in $D\left(\mathfrak{N}_{w}\right)$ Mod

$$
\begin{aligned}
\mathcal{B}_{\mathfrak{X}_{w} \mid \mathfrak{N}_{w}}\left(\mathfrak{N}_{w}\right) & \simeq D\left(\mathfrak{N}_{w}\right) /\left\{D\left(\mathfrak{N}_{w}\right) \operatorname{Dist}^{+}\left(U_{w}^{+}\right)+D\left(\mathfrak{N}_{w}\right) \mathcal{I}_{w}\left(\mathfrak{N}_{w}\right)\right\} \quad \text { by }(2.13) \\
& \simeq \mathfrak{k}\left[U_{w}^{+}\right] \otimes \operatorname{Dist}\left(U_{w}^{-}\right) .
\end{aligned}
$$

On the other hand, we know from [Ke, Lem. 12.8]

$$
\operatorname{ch~} \mathrm{H}_{\mathfrak{X}_{w} / \mathfrak{l} \mathfrak{X}_{w}}\left(\mathfrak{X}, \mathcal{O}_{\mathfrak{X}}\right)=e(w \cdot 0) \prod_{\alpha \in R^{+}} \frac{1}{1-e(-\alpha)} .
$$

As $\operatorname{ch} \mathfrak{k}\left[U^{+}\right] \otimes \operatorname{Dist}\left(U_{w}^{-}\right)=\prod_{\alpha \in R^{+}} \frac{1}{1-e(-\alpha)}$, we must have by the affine version of the Beilinson-Bernstein correspondence (3.9)

$$
\mathcal{B}_{\mathfrak{X}_{w} \mid \mathfrak{N}_{w}}\left(\mathfrak{N}_{w}\right) \simeq \mathfrak{k}\left[U_{w}^{+}\right] \otimes \operatorname{Dist}\left(U_{w}^{-}\right) \otimes(w \cdot 0) \quad \text { in }\left(B_{w}^{+}, D\left(\mathfrak{N}_{w}\right)\right) \operatorname{Mod}
$$


(v) Consider the image of $\operatorname{id}_{\mathfrak{N}_{w}}$ in

$$
D\left(\mathfrak{N}_{w}\right) /\left\{D\left(\mathfrak{N}_{w}\right) \operatorname{Dist}^{+}\left(U_{w}^{+}\right)+D\left(\mathfrak{N}_{w}\right) \mathcal{I}_{w}\left(\mathfrak{N}_{w}\right)\right\} \simeq \mathrm{H}_{\mathfrak{X}_{w} / \mathfrak{l} \mathfrak{X}_{w}}\left(\mathfrak{X}, \mathcal{O}_{\mathfrak{X}}\right) .
$$

As $\mathcal{D}_{\mathfrak{N}_{w}} /\left.\left\{\mathcal{D}_{\mathfrak{N}_{w}} \operatorname{Dist}^{+}\left(U_{w}^{+}\right)+\mathcal{D}_{\mathfrak{N}_{w}} \mathcal{I}_{w}\right\}\right|_{\mathfrak{X}_{w}}=\mathcal{D}_{\mathfrak{N}_{w}} /\left.\mathcal{D}_{\mathfrak{N}_{w}} \operatorname{Dist}^{+}\left(U_{w}^{+}\right)\right|_{\mathfrak{X}_{w}}$ and as $\operatorname{id}_{\mathfrak{N}_{w}}$ is a global section of $\mathcal{H}_{\mathfrak{X}_{w} / \mathfrak{d} \mathfrak{X}_{w}}^{\ell}\left(\mathcal{O}_{\mathfrak{X}}\right)$,

$$
\operatorname{supp}\left(\mathcal{H}_{\overline{\mathfrak{X}_{w}} / \mathfrak{d} \mathfrak{X}_{w}}\left(\mathcal{O}_{\mathfrak{X}}\right)\right) \supseteq \overline{\mathfrak{X}_{w}},
$$

hence $\operatorname{supp}\left(\mathcal{H}_{\overline{\mathfrak{X}_{w}} / \mathfrak{d} \mathfrak{X}_{w}}^{\ell}\left(\mathcal{O}_{\mathfrak{X}}\right)\right)=\overline{\mathfrak{X}_{w}}$ by $\left[\right.$ Ke, Lem. 9.3]. As $\operatorname{id}_{\mathfrak{N}_{w}}$ has weight $w \cdot 0$ and as $\operatorname{dim} \mathrm{H}_{\overline{\mathfrak{X}_{w}} / \mathfrak{l} \mathfrak{X}_{w}}\left(\mathfrak{X}, \mathcal{O}_{\mathfrak{X}}\right)_{w \cdot 0}=1, \operatorname{id}_{\mathfrak{N}_{w}} \in \Gamma(\mathfrak{X}, \mathcal{L}(w))$, hence $\operatorname{supp}(\mathcal{L}(w))=$ $\overline{\mathfrak{X}_{w}}$ also. Finally, as

$$
\left.\mathcal{L}(w)\right|_{\mathfrak{N}_{w}}=\left.\mathcal{H}_{\frac{\ell}{\mathfrak{X}_{w}} / \mathfrak{d} \mathfrak{X}_{w}}\left(\mathcal{O}_{\mathfrak{X}}\right)\right|_{\mathfrak{N}_{w}},\left.\left\{\mathcal{H} \frac{\ell}{\mathfrak{X}_{w} / \mathfrak{d} \mathfrak{X}_{w}}\left(\mathcal{O}_{\mathfrak{X}}\right) / \mathcal{L}(w)\right\}\right|_{\mathfrak{N}_{w}}=0
$$

hence

$$
\operatorname{supp}\left(\mathcal{H}_{\overline{\mathfrak{X}_{w}} / \mathfrak{d} \mathfrak{X}_{w}}^{\ell}\left(\mathcal{O}_{\mathfrak{X}}\right) / \mathcal{L}(w)\right) \subseteq \overline{\mathfrak{X}_{w}} \backslash \mathfrak{N}_{w}=\mathfrak{d} \mathfrak{X}_{w} .
$$

4.2.

Remark. In $[\mathbf{B} \varnothing$, Th. 4.6] Bøgvad goes on to show

$$
\mathcal{L}(w)=\operatorname{soc}_{\left(B^{+}, \mathcal{D}_{X}\right) \mathbf{q c}} \mathcal{H}_{\mathfrak{X}_{w} / \mathfrak{d} \mathfrak{X}_{w}}^{\ell}\left(\mathcal{O}_{\mathfrak{X}}\right)
$$

and that any simple of $\left(B^{+}, \mathcal{D}_{X}\right) \mathbf{q c}$ with support $\overline{\mathfrak{X}_{w}}$ is isomorphic to $\mathcal{L}(w)$. Hence [BBIII, Prop. 2.7] carries over to positive characteristic except that (cf. $[\mathbf{K} 90]$ )

$$
\mathrm{H}_{\mathfrak{X}_{w} / \mathfrak{l} \mathfrak{X}_{w}}\left(\mathfrak{X}, \mathcal{O}_{\mathfrak{X}}\right) \not\left\{\operatorname{Dist}(G) \otimes_{\operatorname{Dist}(B)}(-(w \cdot 0))\right\}^{\star} \quad \text { in } \operatorname{Dist}(G) \operatorname{Mod},
$$

where $\star$ denotes the weightwise dual.

4.3. From $[\mathbf{H} 87,4.4 .1]$ we know

(1) any $\mathcal{M} \in \mathcal{D}_{\mathfrak{X}} \mathbf{q} \mathbf{c}$ is generated by the global sections over $\mathcal{O}_{\mathfrak{X}}$.

Then (cf. [Ka, Th. 1.4.1]) the following three statements are equivalent:

(i) $\mathfrak{X}$ is $\mathcal{D}$-affine,

(ii) for any ample $\mathcal{L} \in \operatorname{Mod}_{\mathfrak{X}}$ if $r \gg 0$, the natural morphism $\mathcal{D}_{\mathfrak{X}} \otimes_{\mathfrak{X}}$ $\left(\mathcal{L}^{\otimes_{-r}} \otimes \mathcal{L}^{\otimes_{r}}(\mathfrak{X})\right) \rightarrow \mathcal{D}_{\mathfrak{X}}$ splits in $\mathbf{A} \mathbf{b}_{\mathfrak{X}}$,

(iii) there is an ample $\mathcal{L} \in \operatorname{Mod}_{\mathfrak{X}}$ such that if $r \gg 0$, the morphism of (ii) splits in $\mathbf{A} \mathbf{b}_{\mathfrak{X}}$. 
If we write $\mathcal{L}=\mathcal{L}(\lambda)=\mathcal{L}_{G / B}(\lambda), \lambda \in \operatorname{Grp}_{\mathfrak{k}}\left(B, \mathrm{GL}_{1}\right)$, the morphism of (ii) is obtained from the commutative diagram in $\left(G, \mathcal{D}_{\mathfrak{X}}\right) \mathbf{q q c}$

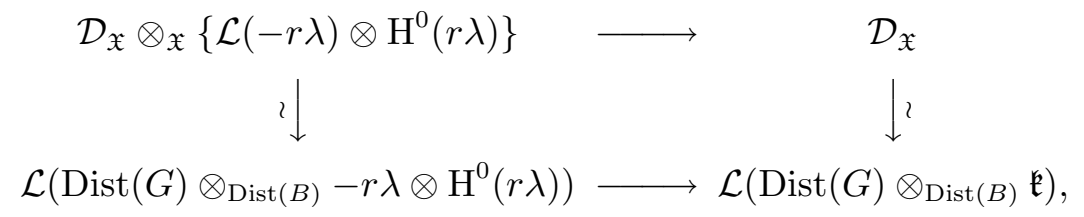

where $\mathrm{H}^{0}(r \lambda)=\mathcal{L}(r \lambda)(\mathfrak{X})$ and the bottom horizontal morphism is induced from the $B$-homomorphism $-r \lambda \otimes \mathrm{ev}_{r \lambda}:-r \lambda \otimes \mathrm{H}^{0}(r \lambda) \rightarrow \mathfrak{k}$ with $\mathrm{ev}_{r \lambda}$ : $\mathrm{H}^{0}(r \lambda) \rightarrow r \lambda$ the evaluation at $e$.

In characteristic $0[\mathbf{B B}]$ finds (cf. [Ka, Th. 6.3.1]) that in (2)

(3) $\operatorname{Dist}(G) \otimes_{\operatorname{Dist}(B)}-r \lambda \otimes \mathrm{H}^{0}(r \lambda) \rightarrow \operatorname{Dist}(G) \otimes_{\operatorname{Dist}(B)} \mathfrak{k}$ splits in $B$ Mod,

hence (ii, iii) hold and the $\mathcal{D}$-affinity of $\mathfrak{X}$ follows. In positive characteristic the statement (ii) is equivalent, given $\mathcal{L}$ and $r$, to the statement that

(4) if $s \gg 0$, the natural morphism $\mathcal{D}_{\mathfrak{X}, s} \otimes_{\mathfrak{X}}\left\{\mathcal{L}^{\otimes_{-r}} \otimes \mathcal{L}^{\otimes_{r}}(\mathfrak{X})\right\} \rightarrow \mathcal{D}_{\mathfrak{X}, s}$ splits in $\mathbf{A} \mathbf{b}_{\mathfrak{X}}$.

If $\mathcal{L}=\mathcal{L}(\lambda)$, the morphism is obtained from the commutative diagram in $\left(G, \mathcal{D}_{\mathfrak{X}, s}\right) \mathbf{q q c}$

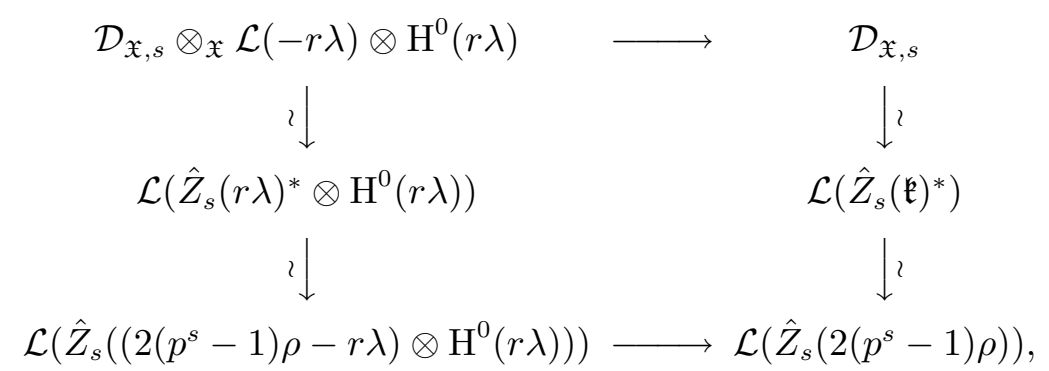

where $\hat{Z}_{s}=\operatorname{ind}_{B}^{G_{s} B}$ and the bottom horizontal morphism is induced from the $G_{s} B$-homomorphism $\theta(s, r \lambda): \hat{Z}_{s}\left(\left(2\left(p^{s}-1\right) \rho-r \lambda\right) \otimes \mathrm{H}^{0}(r \lambda)\right) \rightarrow \hat{Z}_{s}\left(2\left(p^{s}-\right.\right.$ 1) $\rho)$, that in turn is induced from the $B$-homomorphism $\left(2\left(p^{s}-1\right) \rho-r \lambda\right) \otimes$ $\mathrm{ev}_{r \lambda}:\left(2\left(p^{s}-1\right) \rho-r \lambda\right) \otimes \mathrm{H}^{0}(r \lambda) \rightarrow 2\left(p^{s}-1\right) \rho$.

Unfortunately, we find already in $\mathrm{SL}_{2}$ that

$$
\theta\left(s,\left(p^{r}-1\right) \rho\right), s \in \mathbb{N} \text {, does not split in BMod. }
$$

Nevertheless, we can show

$$
\operatorname{id}_{\mathcal{O}_{\mathfrak{X}}} \in \Gamma\left(\mathfrak{X}, \mathcal{L}\left(\theta\left(r+1,\left(p^{r}-1\right) \rho\right)\right)\right),
$$


and hence $G / B$ is $\mathcal{D}$-affine in $\mathrm{SL}_{2}$. More generally, Haastert has proved by different arguments $[\mathbf{H 8 7}, 3.2,4.5 .4]$ that all $\mathbb{P}_{\mathfrak{k}}^{n}, n \in \mathbb{N}$, and the flag variety in $\mathrm{SL}_{3}$ are $\mathcal{D}$-affine.

\section{References}

[BB] A. Beilinson and J. Bernstein, Localisation de $\mathfrak{g}$-modules, C. R. Acad. Sci. Paris, 292 (1981), 15-18.

$[\mathrm{B} \emptyset] \quad \mathrm{R}$. Bøgvad, Some results on $\mathcal{D}$-modules on Borel varieties in characteristic $p>0$, J. Alg., 173 (1995), 638-667.

[BVI] A. Borel, Operations on Algebraic D-modules, 207-270, in A. Borel (ed.) 'Algebraic D-Modules', Academic Press, 1987.

[BBIII] W. Borho and J.-L. Brylinski, Differential operators on homogeneous spaces, III, Inv. Math., 80 (1985), 1-68.

[C] S.U. Chase, On the homological dimension of algebras of differential operators, Comm. Alg., 1 (1974), 351-363.

[DG] M. Demazure and P. Gabriel, Groupes Algébriques, North-Holland, 1970.

[G] R. Godement, Théorie des Faisceaux, Hermann, 1964.

[Gr] P.-P. Grivel, Categories derivees et foncteurs derives, 1-108, in A. Borel (ed.) 'Algebraic D-Modules', Academic Press, 1987.

[EGA0] A. Grothendieck and J. Dieudonné, Éléments de Géométrie Algébrique IV, Pub. Math., 20, IHES, 1964.

[EGAIV] _ Éléments de Géométrie Algébrique IV, Pub. Math., 32, IHES, 1967.

[H86] B. Haastert, Über Differentialoperatoren und $\mathbb{D}$-Moduln in positiver Charakteristik, Dissertation, Univ. Hamburg, 1986

[H87] _ Über Differentialoperatoren und $\mathbb{D}$-Moduln in positiver Charakteristik, Manusc. Math., 58 (1987), 385-415.

[H88] - On direct and inverse images of $\mathcal{D}$-modules in prime charakteristic, Manusc. Math., 62 (1988), 341-354.

[RD] R. Hartshorne, Residues and Duality, LNM 20, Springer-Verlag, 1966.

[H] _ Algebraic Geometry, GTM 52, Springer-Verlag, 1977.

[J] J.C. Jantzen, Representations of Algebraic Groups, Academic Press, 1987.

[K90] M. Kaneda, A note on the Grothendieck-Cousin complex on the flag variety in positive characteristic, Nihonkai Math. J., 1 (1990), 229-251.

[K95] - The Frobenius morphism of Schubert schemes, J. Alg., 174 (1995), 473-488.

[Ka] M. Kashiwara, Representation theory and $\mathcal{D}$-modules on flag varieties, Astérisque, 173-174 (1989), 55-109.

[Ke] G. Kempf, The Grothendieck-Cousin complex of an induced representation, Adv. Math., 29 (1978), 310-396.

[M] H. Matsumura, Commutative Ring Theory, Camb. Univ. Press, 1990.

[MN] Z. Mebkhout and L. Narvaez-Macarro, Sur les coefficients de Rham-Grothendieck des variétés algébriques, 267-308, in F. Baldassarri, S. Bosch and B. Dwork (ed.) ' $p$-adic Analysis', LNM 1454, Springer-Verlag, 1990. 
[Sa] M. Saito, A localization theorem for D-modules, Tôhoku Math. J., 43 (1991), 213234 .

[Sm] S.P. Smith, The global homological dimension of the ring of differential operators on a nonsingular variety over a field of positive characteristic, J. Alg., 107 (1987), 98-105.

[Sp] T. Springer, Linear Algebraic Groups, Birkhäuser, 1981.

[TH] T. Tanisaki and R. Hotta, D-kagun to Daisuugun, Springer-Verlag, 1995.

Received September 12, 1996 and revised April 28, 1997.

OSAKA City UNIVERSity

558 OSAKa SUmiYoshi-KU

Sugimoto, JAPAN

E-mail address: kaneda@sci.osaka-cu.ac.jp

Note: The equation numbers in Sections 2.1, 3.1, and 4.1 of the Paper version WERE INCORRECT. IN ADDITION, REFERENCES TO (2.7.x) HAVE BEEN CHANGED TO (2.8. $x$ ) FOR $x \geq 5$, (2.8.i) HAS BEEN CHANGED TO (2.9.i) IN LINE -2 OF (2.11), AND CORRECTIONS HAVE BEEN MADE IN LINE 1 OF (2.4), IN THE PARAGRAPH AFTER 2.5.8, IN Line 2 of (2.7) Proposition, in the SECONd TERM of (2.7.3), ANd IN (2.9.i). 